\title{
Guidelines for the management of adult lower respiratory tract infections
}

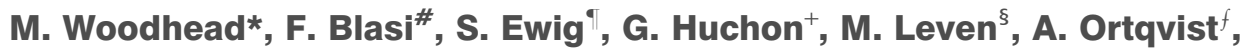

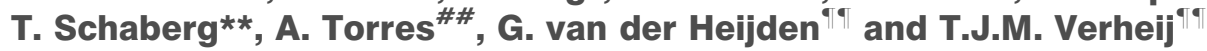

\section{CONTENTS}

\section{Background}

How were the antibiotic recommendations developed

Recommendation summary

Management outside hospital

Diagnosis

Treatment

Management inside hospital

Community-acquired pneumonia.

Exacerbations of COPD

Prevention

Prevention by methods other than vaccination

Prevention by vaccination.

Management outside hospital

Diagnosis

Treatment.

Management inside hospital

Community-acquired pneumonia

Exacerbations of COPD.

Exacerbations of bronchiectasis

Prevention

Prevention by methods other than vaccination.

Prevention by vaccination

Recommendations for influenza vaccination

Recommendations for pneumococcal vaccination

Vaccine uptake

References.

\section{BACKGROUND}

Since the 1998 European Respiratory Society (ERS) lower respiratory tract infection (LRTI) guidelines [1] were published, the evidence on which they were based has increased and the methods for guideline development have been refined. Against this background, these new guidelines have been developed.

A systematic literature search was performed to retrieve relevant publications from 1966 through to December 31, 2002, which critically appraised and rated the pertinent clinical evidence,

For editorial comments see page 979.

s $\ldots \ldots \ldots \ldots \ldots \ldots \ldots \ldots \ldots \ldots \ldots$

AFFILIATIONS

*Dept Respiratory Medicine Manchester Royal Infirmary, Manchester, UK.

${ }^{\#}$ Istituto di Tisiologia e Malattie dell'Apparato Respiratorio, Università degli Studi di Milano, IRCCS Ospedale Maggiore di Milano, Milan Italy.

'Chefarzt der Klinik für Pneumologie Beatmungsmedizin und Infektiologie, Bochum, and,

**Lungenlinik Unterstedt, Daikonierkrankenhaue Rotenburg, Rotenburg, Germany.

+Pneumologie et Reanimation, Paris, France.

${ }^{\S}$ Microbiology Lab, University Hospital Antwerp, Antwerp, Belgium. ${ }^{f}$ Smittskyddsenheten, Norrbacka, Stockholm, Sweden.

\#\# Clinic de Pneumologia I Cirurgia Toràcica, Hospital Clinic I Provincial de Barcelona, Barcelona, Spain.

"Julius Center for Health Sciences and Primary Care, Utrecht, the Netherlands.

\section{CORRESPONDENCE}

M. Woodhead, Dept of Respiratory Medicine, Manchester Royal Infirmary, Oxford Road, Manchester, M13 9WL, UK. Fax: 441612764989 E-mail:

mark.woodhead@cmmc.nhs.uk

Received:

May 112005

Accepted after revision:

August 162005

The following text is a summary of the recommendations themselves and a discussion of the evidence on which the recommendations are based, under the following sections: Management outside hospital; Management inside hospital for community-acquired pneumonia; Exacerbations of COPD; and Exacerbations of bronchiectasis and prevention of infection. These sections, together with full methodological details, definitions, background information regarding descriptive epidemiology, microbiology, risk
SUPPORT STATEMENT

The Task Force of the European Respiratory Society is presented in collaboration with the European Society for Clinical Microbiology and Infectious Diseases (ESCMID).

European Respiratory Journal Print ISSN 0903-1936

Online ISSN 1399-3003 
factors, antimicrobial pharmacodynamics and pharmacokinetics and tables of evidence grading can be found in Appendices 1-3 which are available on the ERS (www.ersnet.org/guidelines) and European Society for Clinical Microbiology and Infectious Diseases (www.escmid. org) websites. The reader is strongly advised to view these.

\section{How were the antibiotic recommendations developed?}

The formulation of the antibiotic recommendations merits specific comment. As with other recommendations, these were based on evidence of both benefit and harm with respect to particular antibiotics. However, robust evidence to support individual recommendations was found to be absent. This was partly because individual antibiotic studies do not capture all outcomes of importance in antibiotic management and also because there may be variation in factors, such as the prevalence of antibiotic resistance of leading pathogens like Streptococcus pneumoniae, that might determine antibiotic recommendation in different geographical locations which cannot be addressed by a single recommendation. Bacterial antibiotic resistance is common in some countries, but its clinical relevance is often unclear. Factors such as, lack of statistical power to assess an outcome, selective patient recruitment, lack of subject blinding, and lack of assessment of impact on the wider community (especially with regard to antimicrobial resistance), were common to most clinical studies of antibiotic effect. Use of such studies could, therefore, only be used to support a consensus view from the Guideline authors.

The antibiotic recommendations should be interpreted with the above in mind and it should be accepted that an individual recommendation may not be suitable in every clinical setting. When an antibiotic is stated as "preferred" this should be taken to mean that in the view of the authors, based on available evidence, in usual everyday management, this antibiotic would have advantages over others. This is not to say that other antibiotics might not be effective and in some, usually less common, situations might even be preferred.

\section{RECOMMENDATION SUMMARY Management outside hospital}

Diagnosis

When should aspiration pneumonia be considered?

In patients with difficulties with swallowing who show signs of an acute LRTI. In these patients a chest radiograph should be performed (C3).

When should cardiac failure be considered?

In patients aged $>65 \mathrm{yrs}$, with orthopnoea, displaced apex beat and/or a history of myocardial infarction (C3).

\section{When should pulmonary embolism be considered?}

In patients with one of the following characteristics: a history of deep vein thrombosis (DVT) or pulmonary embolism; immobilisation in past 4 weeks; or malignant disease (C3).

\section{When should chronic airway disease be considered?}

In patients with at least two of the following: wheezing; prolonged expiration; history of smoking; and symptoms of allergy. Lung-function tests should be considered to assess the presence of chronic lung disease (C3).

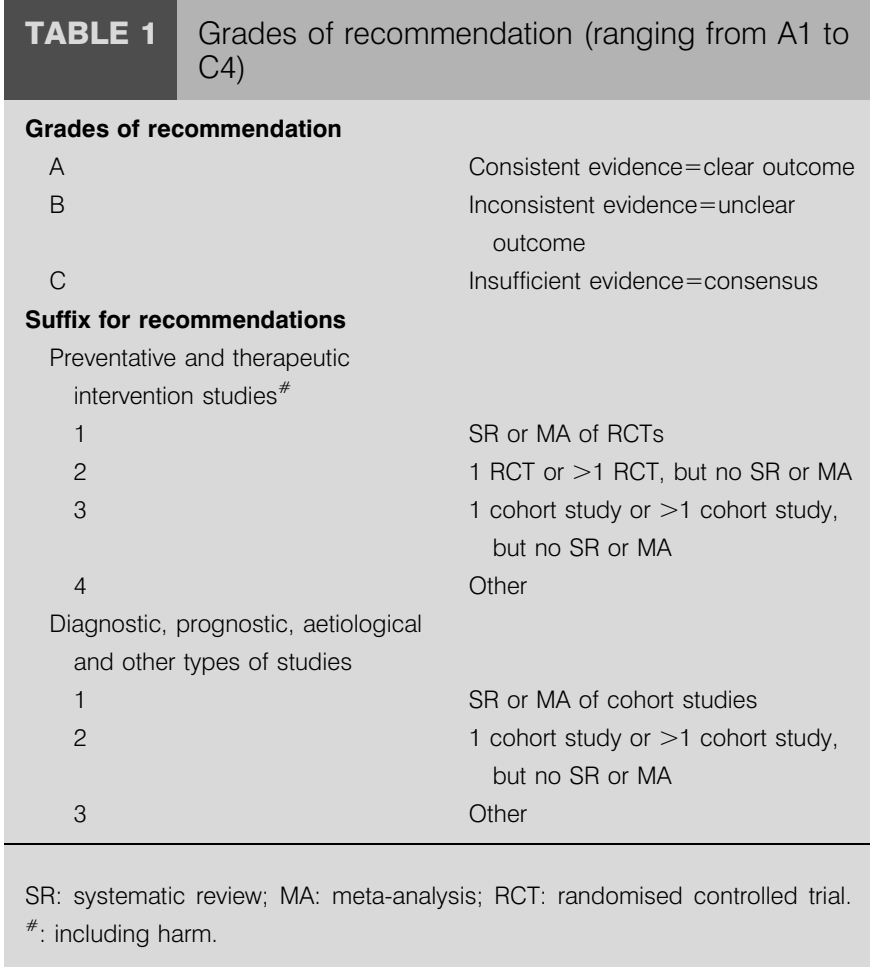

How to differentiate between pneumonia and other respiratory tract infections

A patient should be suspected of having pneumonia when acute cough and one of the following signs/symptoms are present: new focal chest signs; dyspnoea; tachypnoea; fever lasting $>4$ days.

If pneumonia is suspected, a chest radiograph should be performed to confirm the diagnosis (C1).

\section{Should the primary care physician test for a possible microbiological aetiology of LRTI?}

Microbiological investigations are not usually recommended in primary care $(\mathrm{C} 1-\mathrm{C} 3)$.

\section{Treatment}

Should symptomatic acute cough be treated?

Both dextromethorphan and codeine can be prescribed in patients with a dry and bothersome cough (C1). Expectorant, mucolytics, antihistamines and bronchodilators should not be prescribed in acute LRTI in primary care (A1).

\section{When should antibiotic treatment be considered in patients with} LRTI?

Antibiotic treatment should be considered in patients with LRTI in the following situations: suspected or definite pneumonia (see How to differentiate between pneumonia and other respiratory tract infections); selected exacerbations of chronic obstructive pulmonary disease (COPD; see What are the indications for antibiotic treatment of exacerbations of COPD?); aged >75 yrs and fever; cardiac failure; insulindependent diabetes mellitus; and serious neurological disorder (stroke etc.; C2). 
What are the indications for antibiotic treatment of exacerbations of COPD?

An antibiotic should be given during exacerbations of COPD in patients with all three of the following symptoms: increased dyspnoea; increased sputum volume; and increased sputum purulence. In addition, antibiotics should be considered for exacerbations in patients with severe COPD (C1).

\section{Which antibiotics should be used in patients with LRTI?}

Tetracycline and amoxicillin are first-choice antibiotics. In case of hypersensitivity, newer macrolides, such as azithromycin, roxithromycin or clarithromycin, are good alternatives in countries with low pneumococcal macrolide resistance. National/local resistance rates should be considered when choosing a particular antibiotic. When there are clinically relevant bacterial resistance rates against all first-choice agents, treatment with levofloxacin or moxifloxacin may be considered (C4; table 2).

\section{Is anti-viral treatment useful in patients with LRTI?}

The empirical use of anti-viral treatment in patients suspected of suffering from influenza is usually not recommended (B1). Only in high-risk patients who have typical influenza symptoms (fever, muscle ache, general malaise and respiratory tract infection) for $<2$ days, and during a known influenza epidemic, can anti-viral treatment be considered (C1).
How should patients with LRTI be monitored?

A patient should be advised to return if symptoms take $>3$ weeks to disappear.

Clinical effects of the antibiotic treatment should be expected within 3 days and patients should be instructed to contact their doctor if this effect is not noticeable. Seriously ill patients, i.e. having at least two of the following symptoms/characteristics, should already be seen 2 days after the first visit: high fever; tachypnoea; dyspnoea; relevant comorbidity; aged $>65$ yrs.

All patients or persons within their environment should be advised to contact their doctor again if: fever exceeds 4 days; dyspnoea gets worse; patients stop drinking; or consciousness decreases (C3).

\section{Management inside hospital}

Community-acquired pneumonia

Who should be admitted to hospital?

The decision to hospitalise remains a clinical decision. However, this decision should be validated against at least one objective tool of risk assessment. Both the pneumonia severity index (PSI) and the CURB index (mental confusion, urea, respiratory rate, blood pressure; see later sections) are valid tools in this regard. In patients with a PSI of IV and V, and/or a CURB of $\geqslant 2$, hospitalisation should be seriously considered (A3).

\section{TABLE 2 Summary of antibiotic recommendations ${ }^{\#}$}

\begin{tabular}{|c|c|c|c|c|}
\hline \multirow[t]{2}{*}{ Setting } & \multirow[t]{2}{*}{ LRTI type } & \multirow[t]{2}{*}{ Severity/sub-group } & \multicolumn{2}{|r|}{ Treatment } \\
\hline & & & Preferred & Alternative \\
\hline Community & $\mathrm{LRTI}^{+}$ & All & Amoxicillin or tetracyclines ${ }^{\S}$ & Co-amoxiclav, macrolide ${ }^{f}$, levofloxacin, moxifloxacin \\
\hline Hospital & $\mathrm{COPD}^{+}$ & Mild & Amoxicillin or tetracyclines ${ }^{\S}$ & Co-amoxiclav, macrolide ${ }^{f}$, levofloxacin, moxifloxacin \\
\hline Hospital & COPD & Moderate/severe & Co-amoxiclav & levofloxacin, moxifloxacin \\
\hline Hospital & CAP & Nonsevere & $\begin{array}{l}\text { Penicillin } \mathrm{G} \pm \text { macrolide } \\
\text { aminopenicillin } \pm \text { macrolide } \\
\text { co-amoxiclav } \pm \text { macrolide } ; \text {; } 2 \text { nd } \\
\text { or 3rd cephalosporin } \pm \text { macrolide } f\end{array}$ & levofloxacin, moxifloxacin \\
\hline Hospital & CAP & Severe & 3rd Cephalosporin + macrolide ${ }^{f}$ & 3rd Cephalosporin + (levofloxacin or moxifloxacin) \\
\hline Hospital & CAP & $\begin{array}{l}\text { Severe and risk factors } \\
\quad \text { for } P \text {. aeruginosa }\end{array}$ & $\begin{array}{l}\text { Anti-pseudomonal cephalosporin } \\
++ \text { ciprofloxacin }\end{array}$ & $\begin{array}{c}\text { Acylureidopenpenicillin/ } \beta \text {-lactamase } \\
\text { inhibitor }+ \text { ciprofloxacin or } \\
\text { Carbapenem }+ \text { ciprofloxacin }\end{array}$ \\
\hline
\end{tabular}

LRTI: lower respiratory tract infection; COPD: chronic obstructive pulmonary disease; CAP: community-acquired pneumonia; P. aeruginosa: Pseudomonas aeurginosa. \#: see introductory paragraphs for derivation of terms used; " to be used in the presence of hypersensitivity to preferred drug or widespread prevalence of clinically relevant resistance in the population being treated. In some European countries only "alternatives" will be used; ${ }^{+}$: antibiotic therapy may not be required (see text for indications for antibiotic therapy); ${ }^{\text {s: }}$ tetracycline or doxycycline; f: erythromycin, clarithromycin, roxithromycin or azithromycin. Telithromycin may be an alternative for consideration in the community or in hospitals for COPD exacerbation or CAP. However, clinical experience with this antibiotic is currently too limited to make specific recommendations. Oral cephalosporins are generally not recommended due to poor pharmacokinetics. For recommended dosages see Appendix 3 
Additional requirements of patient management, as well as social factors not related to pneumonia severity, must be considered as well.

Who should be considered for intensive care unit admission? Criteria of acute respiratory failure, severe sepsis or septic shock and radiographic extension of infiltrates should prompt consideration of the admission to the intensive care unit (ICU) or an intermediate care unit.

The presence of at least two of the following indicates severe community-acquired pneumonia (CAP) and can be used to guide ICU referral: systolic blood pressure $<90 \mathrm{mmHg}$; severe respiratory failure (arterial oxygen tension $\left(\mathrm{Pa}_{\mathrm{a}} \mathrm{O}_{2}\right)$ /inspiratory oxygen fraction $\left.\left(\mathrm{FI}_{1} \mathrm{O}_{2}\right)<250\right)$; involvement of more than two lobes on a chest radiograph (multilobar involvement); requirement for either mechanical ventilation or requirement of vasopressors $>4 \mathrm{~h}$ (septic shock; A3).

What laboratory studies should be performed?

The amount of laboratory and microbiological work-up should be determined by the severity of pneumonia (A3).

What is the value of blood cultures in the diagnosis of CAP? Blood cultures should be performed in all patients with CAP who require hospitalisation (A3).

What other invasive techniques for normally sterile specimens can be useful in the laboratory diagnosis of pneumonia?

The following invasive techniques can be useful in laboratory diagnosis. 1) Diagnostic thoracentesis should be performed when a significant pleural effusion is present (A3). 2) Due to the inherent potential adverse effects, trans-thoracic needle aspiration can only be considered on an individual basis for some severely ill patients with a focal infiltrate, in whom less invasive measures have been nondiagnostic (A3). 3) Bronchoscopic protected specimen brush (PSB) and bronchoalveolar lavage (BAL). BAL may be the preferred technique in nonresolving pneumonia (A3). Bronchoscopic sampling of the lower respiratory tract can be considered in intubated patients and selected nonintubated patients where gas exchange status allows (A3).

What is the value of sputum examination?

Gram stain is recommended when a purulent sputum sample can be obtained from patients with $\mathrm{CAP}$ and is processed timely (A3).

A culture from a purulent sputum specimen of a bacterial species compatible with the morphotype observed in the Gram stain, which is processed correctly, is worthwhile for confirmation of the species identification and antibiotic susceptibility testing (B3).

What can antigen tests offer in the diagnosis of CAP?

Legionella pneumophila serogroup 1 antigen detection in urine is recommended for patients with severe CAP and in other patients where this infection is clinically or epidemiologically suspected (A3).

What can serological tests offer in the diagnosis of pneumonia? Serological tests for the management of the individual patient with CAP are not recommended (A3).
Serology for infections caused by Mycoplasma pneumoniae, Chlamydia pneumoniae and Legionella is more useful in epidemiological studies rather than in the routine management of the individual patient (A3).

Are amplification tests useful for the diagnosis of CAP?

Application of molecular tests for the detection of influenza and respiratory syncytial virus (RSV) may be considered during the winter season, and for the detection of atypical pathogens provided the tests are validated and the results can be obtained sufficiently rapidly to be therapeutically relevant (A3).

\section{What classification should be used for treatment?}

Antimicrobial treatment has to be empiric and should follow an approach according to the individual risk of mortality. The assessment of severity according to mild, moderate and severe pneumonia implies a decision regarding the most appropriate treatment setting (ambulatory, hospital ward, ICU; A4). Antimicrobial treatment should be initiated as soon as possible (A3).

The guidance of empiric initial antimicrobial treatment should follow: 1) general patterns of expected pathogens according to pneumonia severity and additional risk factors; 2) regional and local patterns of microbial resistance; 3) considerations of tolerability and toxicity of antimicrobial agents in the individual patient.

What initial empiric treatments are recommended?

The treatment options for hospitalised patients with moderate and severe CAP are shown in tables 3 and 4, respectively.

\section{What is the recommended treatment for specific identified} pathogens?

For details see the later section entitled What is the recommended treatment for specific identified pathogens?, in the main body of the text.

\section{What should be the duration of treatment?}

The appropriate duration of antimicrobial treatment has not been settled. In comparative studies, the usual duration of treatment is $\sim 7-10$ days. Intracellular pathogens, such as Legionella spp. should be treated for at least 14 days (C4).

\section{When should i.v. be used and when should the switch to oral} occur?

In mild pneumonia, treatment can be applied orally from the beginning (A3). In patients with moderate pneumonia, sequential treatment should be considered in all patients except the most severely ill. The optimal time to switch to oral treatment is also unknown; it seems reasonable to target this decision according to the resolution of the most prominent clinical features upon admission (A3).

\section{Which additional therapies are recommended?}

Low molecular heparin is indicated in patients with acute respiratory failure (A3). The use of noninvasive ventilation is not yet a standard of care, but may be considered particularly in patients with COPD (B3). The treatment of severe sepsis and septic shock is confined to supportive measures (A3). Steroids 


\begin{tabular}{|c|c|c|}
\hline TABLE 3 & \multicolumn{2}{|c|}{$\begin{array}{l}\text { Preferred and alternative treatment options (in no } \\
\text { special order) for hospitalised patients with } \\
\text { moderate community-acquired pneumonia (C4) }\end{array}$} \\
\hline Preferred $^{\#}$ & & Alternative \\
\hline \multicolumn{2}{|c|}{ 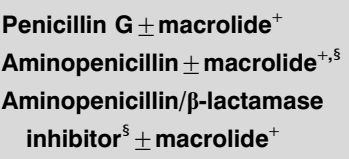 } & $\begin{array}{l}\text { Levofloxacin } \\
\text { Moxifloxacin }\end{array}$ \\
\hline \multicolumn{3}{|c|}{ 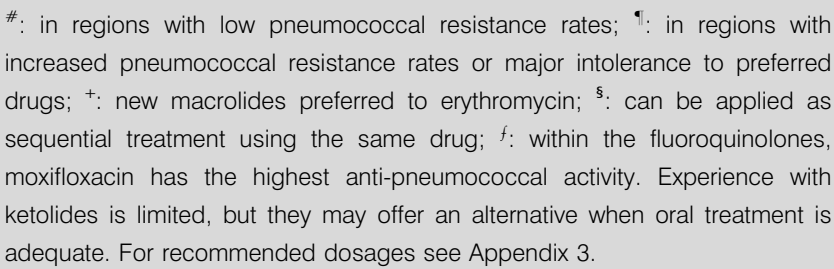 } \\
\hline
\end{tabular}

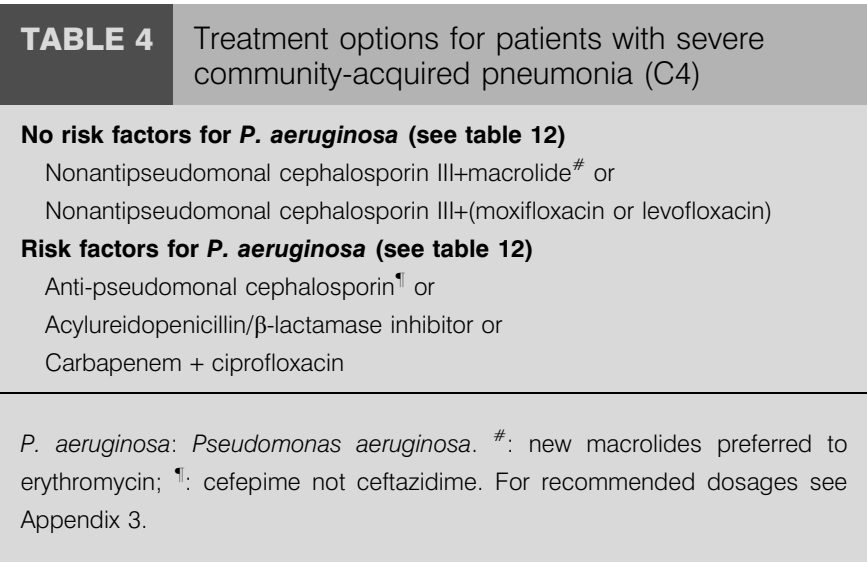

have no place in the treatment of pneumonia unless septic shock is present (A3).

\section{How should response be assessed and when should chest} radiographs be repeated?

Response to treatment should be monitored by simple, clinical criteria including body temperature, respiratory and haemodynamic parameters. The same parameters should be applied to judge the ability of hospital discharge (A3). Complete response, including radiographic resolution, requires longer time periods. Discharge decisions should be based on robust markers of clinical stabilisation (A3).

\section{How should the nonresponding patient be assessed?}

The two types of treatment failures, nonresponding pneumonia and slowly resolving pneumonia, should be differentiated (A3). The evaluation of nonresponding pneumonia depends on the clinical condition. In unstable patients, full reinvestigation followed by a second empiric antimicrobial treatment regimen is recommended. The latter may be withheld in stable patients. Slowly resolving pneumonia should be reinvestigated according to clinical needs in relation to the condition and individual risk factors of the patient $(\mathrm{C} 3)$.

\section{Exacerbations of COPD \\ Which hospitalised patients with COPD exacerbations should receive antibiotics?}

The following hospitalised patients with COPD should receive antibiotics. 1) Patients with all three of the following symptoms: increased dyspnoea; sputum volume; and sputum purulence (a Type I Anthonisen exacerbation; A2). 2) Patients with only two out of the three symptoms above (a Type II Anthonisen exacerbation) when increased purulence of sputum is one of the two cardinal symptoms (A2). 3) Patients with a severe exacerbation that requires invasive or noninvasive mechanical ventilation (A2). 4) Antibiotics are generally not recommended in Anthonisen Type II without purulence and Type III patients (one or none of the above symptoms; A2).

\section{What stratification of patients with COPD exacerbation is recommended to direct treatment?}

The following groups of COPD patients are recommended to direct treatment. Group A: patients not requiring hospitalisation (mild COPD, see Management outside hospital; A3). Group B: admitted to hospital (moderate-severe COPD) without risk factors for Pseudomonas aeruginosa infection (A3). Group C: admitted to hospital (moderate-severe COPD) with risk factors for $P$. aeruginosa (A3).

\section{What are the risk factors for $\mathrm{P}$. aeruginosa?}

At least two out of the following four are risk factors for $P$. aeruginosa: 1 ) recent hospitalisation (A3); 2) frequent (more than four courses per year) or recent administration of antibiotics (last 3 months; A3); 3) severe disease (forced expiratory volume in one second (FEV1) $<30 \%$; $\mathrm{A} 3$ ); 4) previous isolation of $P$. aeruginosa during an exacerbation or patient colonised by $P$. aeruginosa (A3).

\section{Which microbiological investigations are recommended for hospitalised patients with COPD exacerbation?}

In patients with severe exacerbations of COPD (Group C patients), difficult to treat microorganisms ( $P$. aeruginosa) or potential resistances to antibiotics (prior antibiotic or oral steroid treatment, prolonged course of the disease, more than four exacerbations per year and FEV1 $<30 \%$ ), sputum cultures or endotracheal aspirates (in mechanically ventilated patients) are recommended (A3).

\section{Which initial antimicrobial treatments are recommended for} patients admitted to hospital with COPD exacerbation? In patients without risk factors for $P$. aeruginosa, several options for antibiotic treatment are available. The selection of one or other antibiotic depends on the severity of the exacerbation, local pattern of resistances, tolerability, cost and potential compliance. Amoxicillin or tetracycline is recommended for mild exacerbations (which might usually be managed at home) and co-amoxiclav for those admitted to hospital with moderate-severe exacerbations (A2).

In patients with risk factors for $P$. aeruginosa, ciprofloxacin is the antibiotic of choice when the oral route is available. When 
parenteral treatment is needed, ciprofloxacin or a $\beta$-lactam with anti-pseudomonal activity are available options. The addition of aminoglycosides is optional (A2).

The use of the oral or i.v. route depends on the stability of the clinical condition and the severity of exacerbation. Switch (i.v. to oral) is recommendable by day 3 of admission if the patient is clinically stable. (A3)

How should the nonresponding patient with COPD exacerbation be assessed?

After close re-evaluation of noninfectious causes of failure (i.e. inadequate medical treatment, embolisms, cardiac failure, other), a careful microbiological reassessment, as mentioned in the microbiological diagnosis section, is recommended (C3).

The recommendation for treatment in cases of failure includes an antibiotic change with good coverage against $P$. aeruginosa S. pneumoniae resistant to antibiotics and nonfermenters and to subsequently adjust the new antibiotic treatment according to microbiological results (C3).

\section{General recommendations for exacerbations of bronchiectasis}

The general recommendations are as follows. 1) Periodical surveillance of colonisation is advisable (B3), frequency must be indicated. 2) The majority of patients with exacerbations will benefit from antibiotic treatment (B3). 3) Obtaining a sputum sample for culture before starting antibiotic treatment is recommended in most cases and particularly in those requiring hospitalisation (B3). 4) For empirical antibiotic treatment patients have to be stratified according to the potential risk of Pseudomonas spp. infection (B3). Recommended antibiotics are summarised in table 5. Empirical antibiotics have to be adjusted or modified according to sputum culture results (A3).

\section{Prevention}

Prevention by methods other than vaccination

Does oral immunisation with bacterial extracts prevent LRTI?

The use of the Haemophilus influenzae oral vaccine (B1) or bacterial extracts (OM-85 BV; B 2) in patients with chronic bronchitis (CB) or COPD is not recommended.

\begin{tabular}{|c|c|c|}
\hline \multirow[t]{2}{*}{ TABLE 5} & \multicolumn{2}{|c|}{$\begin{array}{l}\text { Antibiotics recommended for exacerbations of } \\
\text { bronchiectasis (C4) }\end{array}$} \\
\hline & Oral treatment & Parenteral treatment \\
\hline \multirow[t]{5}{*}{$\begin{array}{l}\text { No risk of } \\
\text { Pseudomonas spp. }\end{array}$} & Amoxicillin-clavulanate & Amoxicillin-clavulanate \\
\hline & Moxifloxacin & Ceftriaxone \\
\hline & Levofloxacin & Cefotaxime \\
\hline & & Moxifloxacin \\
\hline & & Levofloxacin \\
\hline $\begin{array}{l}\text { Risk of Pseudomonas } \\
\text { spp.\# }\end{array}$ & Ciprofloxacin & $\begin{array}{l}\text { Ciprofloxacin }+ \\
\text { anti-pseudomonal } \\
\quad \beta \text {-lactam } \\
\text { or aminoglycoside }\end{array}$ \\
\hline \multicolumn{3}{|c|}{$\begin{array}{l}\text { \#: use the same criteria mentioned for chronic obstructive pulmonary disease } \\
\text { exacerbation; }{ }^{\bullet} \text { : ceftazidime, cefepime, carbapenems, piperacillin-tazobactam. } \\
\text { For recommended dosages see Appendix } 3 \text {. }\end{array}$} \\
\hline
\end{tabular}

What is the role of prophylactic antibiotic therapy in $C B$ or COPD? The prophylactic use of antibiotics in patients with $C B$ or COPD as a matter of prevention is not recommended (A1).

According to the opinion of experts, it might be justified to use long-term antibiotic therapy in selected patients with bronchiectasis who suffer from frequent bacterial exacerbations, but no data from controlled studies are available for an evidencebased recommendation (C4).

The use of nebulised antibiotics for the prevention of LRTI in patients with bronchiectasis has not been studied systematically so far. Therefore, no evidence for its use could be found and the use of this approach is not recommended (C4).

\section{Does antibiotic treatment of upper respiratory tract infections prevent $L R T I$ ?}

Treatment of upper respiratory tract infections (URTI) with antibiotics will not prevent LRTI (A1).

\section{Does treatment with inhaled steroids or long-acting $\beta_{2}$-agonists} prevent LRTI?

The regular use of inhaled steroids (B1) or of long-acting $\beta_{2^{-}}$ agonists (C4) as preventive approaches for LRTI is not recommended. This does not mean that they might not prevent exacerbations of COPD, which is an issue beyond the scope of this document.

\section{Does regular physiotherapy prevent LRTI?}

Physiotherapy is not recommended as a preventive approach against LRTI (C4).

\section{Do anti-viral substances prevent influenza virus infection?}

Prevention of influenza by anti-viral substances is only recommended in unusual situations (for example, in outbreaks within closed communities; A1).

Are oral mucolytics useful for the prevention of LRTI?

The regular use of oral mucolytics in patients with CB and COPD as a matter of prevention against LRTI is not recommended (B1). The regular use of oral mucolytics in patients with bronchiectasis as a matter of prevention against LRTI is also not recommended (B1).

Is there evidence that homeopathic substances prevent LRTI? The use of homeopathic substances is not recommended as a preventive approach against LRTI (C4).

\section{Prevention by vaccination}

Should the influenza vaccine be used to prevent LRTI?

For influenza vaccination, the following are recommended. 1) The influenza vaccine should be given yearly to those at increased risk for complications due to influenza (A1). The vaccination is recommended for immunocompetent adults belonging to one, or more, of the following categories: aged $\geqslant 65$ yrs; institutionalisation; chronic cardiac diseases; chronic pulmonary diseases; diabetes mellitus; chronic renal diseases; haemoglobinopathies; and females who will be in the second or third trimester of pregnancy during the influenza season. 2) Repeated vaccinations are safe and do not lead to a decreased immune response (B1). 3) In adults an inactivated, rather than live attenuated, vaccine is recommended (A1). 4) In healthcare 
personnel yearly vaccination is recommended, especially in settings where elderly persons or other high-risk groups are treated (B2).

\section{Should pneumococcal vaccine be used to prevent $L R T I$ ?}

For pneumococcal vaccination the following are recommended. 1) The evidence for vaccination with the 23-valent polysaccharide pneumococcal vaccine is not as strong as that for the influenza vaccination, but it is recommend that the vaccine be given to all adult persons at risk from pneumococcal disease (B4). 2) Risk factors for pneumococcal disease are: age $\geqslant 65 \mathrm{yrs}$; institutionalisation; dementia; seizure disorders; congestive heart failure; cerebrovascular disease; COPD; history of a previous pneumonia; chronic liver disease; diabetes mellitus; functional or anatomic asplenia; and chronic cerebrospinal fluid leakage (B3). Although smoking seems to be a significant risk factor in otherwise healthy, younger adults, measures aimed at reducing smoking and exposure to environmental tobacco smoke should be preferred in this group. 3) Revaccination, once, can be considered in the elderly, 5-10 yrs after primary vaccination (B3).

What is the best way to implement influenza and pneumococcal vaccination policies?

Active interventions to enhance vaccination with either or both vaccines are effective and needed to achieve adequate vaccination coverage of the targeted population (B1).

\section{MANAGEMENT OUTSIDE HOSPITAL \\ Diagnosis}

Outpatients contact general practitioners (GPs) with complaints, such as coughing and dyspnoea, but not with diagnoses, such as acute bronchitis, asthma or pneumonia. This section includes signs and symptoms as a starting point, particularly symptoms indicating that the patient has a disorder of the lower airways, e.g. cough, dyspnoea, wheezing, coughing up sputum, pain in the chest etc. Some of these symptoms are more related than others to LRTI, see the Definitions section (Appendix 1).

There are three important diagnostic issues concerning LRTI in daily primary care practice. First, it is important to assess whether the symptoms of the patient are actually caused by an infection or by another noninfectious disorder, such as asthma, COPD, heart failure or lung infarction. Secondly, if it is likely that the patient has a respiratory tract infection, what part of the respiratory tract is affected? Does the patient have an acute bronchitis or is it pneumonia? Thirdly, the GP would ideally like information regarding the nature of the microbiological pathogen(s) involved. Does the patient have a viral or bacterial infection, or both, and which viruses and bacteria are involved? The word "ideally" is used here because testing for the presence of pathogens involved is often not useful.

\section{When should aspiration pneumonia be considered?}

Aspiration should be excluded, especially in patients who have difficulties with swallowing, for instance, after cerebral vascular events and in certain psychiatric diseases. There are, however, no studies to support this expert opinion.

\section{Recommendation}

Aspiration pneumonia should be considered in patients who have difficulties with swallowing and who show signs of an acute LRTI. In these patients a chest radiograph should be performed (C3).

\section{When should cardiac failure be considered?}

Cardiac failure might be very difficult to detect. There are few studies available on its diagnosis in primary care. A history of myocardial infarction and a finding of a displaced apex beat were the best predictors of left ventricular dysfunction in a study of 259 patients with suspected cardiac failure [2]. Another study showed older age, male sex, orthopnoea, a history of myocardial infarction and absence of COPD to be predictors of cardiac failure [3].

\section{Recommendation}

Cardiac failure should be considered in patients aged $>65$ yrs with either orthopnoea, displaced apex beat and/or a history of myocardial infarction (C3).

\section{When should pulmonary embolism be considered?}

Pulmonary embolism (PE) can present with a simple acute cough and be difficult to discern from an LRTI. The absence of signs of DVT, immobilisation in the past 4 weeks, a history of DVT or PE, haemoptysis, pulse $>100$ and malignant disease, shows that PE is highly unlikely [4].

\section{Recommendation}

PE should be considered in patients with one of the following characteristics: a history of DVT or pulmonary embolism; immobilisation in past 4 weeks; or malignant disease (C3).

\section{When should chronic airways disease be considered?}

Chronic lung disorders, such as asthma and COPD, can also present exacerbations with symptoms, such as coughing, sputum and dyspnoea. The few available studies show that a considerable portion of patients with an acute cough or a diagnosis of acute bronchitis do in fact have asthma or COPD (up to $45 \%$ in patients with an acute cough $>2$ weeks) [5-7]. Wheezing, prolonged expiration, number of pack-yrs, a history of allergy and female sex appear to have predictive values for the presence of asthma/COPD [5]. This is relevant because lung medication, e.g. $\beta$-agonists and steroids, have been shown to be beneficial in these exacerbations.

\section{Recommendation}

Lung function tests should be considered to assess the presence of chronic lung disease in patients with at least two of the following signs: wheezing; prolonged expiration; history of smoking; and symptoms of allergy (C3).

There is, however, still considerable discussion as to whether exacerbations of asthma and COPD are in fact viral or bacterial LRTIs in such patients. Viral respiratory tract infections can trigger exacerbations, but there is controversy as to whether a microbiological infection is a clinically relevant phenomenon during exacerbation. The implications of this uncertainty for daily practice will be discussed in the Treatment section. 
How to differentiate between pneumonia and other respiratory tract infections

Respiratory symptoms, such as cough and dyspnoea, can be caused by inflammation of the trachea, bronchi, bronchioli and the lung parenchyma. There are also cough receptors in the upper respiratory tract and, thus, cough can also be caused by URTIs. However, whether URTIs are a frequent cause of acute cough is uncertain. Studies into the relationship between sinusitis and cough, for instance, are only carried out in patients with chronic cough and suffer from methodological flaws. Hence, there is no evidence supporting the widely accepted concept of post-nasal drip being an important cause of acute cough [8-10]. This does not mean that patients with a URTI cannot have an LRTI at the same time.

Differentiating between tracheitis and acute bronchitis is impossible in daily practice and not relevant. Usually these two entities are taken together and often acute tracheobronchitis is only referred to as acute bronchitis. Differentiating between acute bronchitis and pneumonia is, on the other hand, important. Pneumonia is a more severe infection than acute bronchitis with a higher risk for complications and prolonged course of symptoms.

The gold standard for the diagnosis of pneumonia is a chest radiograph. However, LRTI symptoms reported to the GP are extremely common (100 per 1,000 persons $\cdot \mathrm{yr}^{-1}$ ), and only 5$10 \%$ of these patients have pneumonia. This means that it is neither feasible nor cost-effective to perform radiological tests in all patients with lower respiratory tract symptoms.

There are several studies on the diagnostic value of signs and symptoms for the presence of an infiltrate on the chest radiograph. However, interpretation of the results of these studies is difficult because of the low numbers of patients with pneumonia who are included, thus, encountering the problem that signs, like a dull percussion note or a pleural rub, are only present in a minority of patients with pneumonia; if present, a pneumonia is very likely, but absence of these signs will not make the GP any wiser. Focal chest signs perhaps are more helpful. One study found that in patients with focal auscultatory abnormalities, 39\% did have pneumonia as opposed to 5$10 \%$ in all patients with an acute cough. In patients without focal signs the probability of $5-10 \%$ was reduced to $2 \%$ [11]. Only a few studies also looked at the diagnostic value of combinations of signs and symptoms as physicians always do in daily practice. Fever, absence of URTI symptoms, dyspnoea/tachypnoea and abnormal chest signs were usually present in these models [12]. None of these algorithms have been properly validated in other populations.

There is also discussion on the value of additional tests, such as C-reactive protein (CRP). Some studies have shown that an elevated level of CRP in the patient's serum $\left(>50 \mathrm{mg} \cdot \mathrm{mL}^{-1}\right)$ could increase the chance that the patient involved does have pneumonia $[13,14]$. Sufficient data on the additional diagnostic value of $C R P$, next to history and physical examination, are not yet available.

Based on the discussion in the literature and the expertise of the members of the ERS Task Force the following diagnostic strategy is advocated.

\section{Recommendation}

A patient should be suspected of having pneumonia when the following signs and symptoms are present.

An acute cough and one of the following: new focal chest signs; dyspnoea; tachypnoea; or fever $>4$ days. If pneumonia is suspected, a chest radiograph should be performed to confirm the diagnosis (C1).

\section{Should the primary care physician test for a possible microbiological aetiology of LRTI?}

The main reason for detecting a microbiological cause of symptoms would be to select patients who could benefit from antibiotic treatment and enable therapy with narrow-spectrum antibiotics to contain bacterial resistance, side-effects and costs. In addition, public health is sometimes served by detection of particular pathogens, such as tuberculosis and legionella. Other important infections, e.g. influenza, are usually monitored by public health authorities. Conversely, one should note that a large proportion of patients with LRTI do not benefit from antimicrobial treatment, irrespective of the aetiology of their disease. Only in certain sub-groups of patients, such as very young children, very old patients and patients with serious chronic comorbidity, e.g. COPD, cardiac failure or diabetes, could assessment of the microbiological aetiology be useful.

Two separate issues should be addressed here: 1) detecting whether the patient has an LRTI of bacterial origin; and 2) testing which species of bacteria are involved and assessing the antibiotic resistance of these possible pathogens.

The colour of expectorated sputum is often said to be related to bacterial LRTI. One study of patients with an exacerbation of COPD showed a clear relationship between purulence and quantity of bacteria in sputum [15]. Whether these findings can be confirmed by others, and if this also applies for patients without chronic lung disease, is still unknown. Serum levels of CRP are also used to assess the presence of a bacterial aetiology. However, the results of studies in this field are equivocal [16-18]. Studies on the diagnostic value of Gram stain in primary care patients with LRTI are lacking. However, hospital-based studies on the use of Gram stain in CAP show low sensitivity for detecting possible pathogens [19]. Bacterial colonisation (as opposed to infection) was not taken into account in these studies. It is unlikely that this test performs better in primary care, where, on average patients, have milder disease forms.

The reasons for not advocating Gram stain also apply to culturing sputum samples and measuring pneumococcal antigen in sputum and urine. Possible bacterial pathogens are only detected in $20-50 \%$ of patients and a distinction between colonisation and a new bacterial infection is difficult.

\section{Recommendation}

Microbiological investigations are not usually recommended in primary care. Differentiating between viral and bacterial infections is difficult in primary care patients. Indications for treatment should, therefore, be based on assessment of severity of the clinical syndrome (see Treatment section). Also, the 
physician should be aware of local bacterial resistance rates (C1-3).

\section{Treatment}

Most episodes of LRTI are self-limiting and will last between 1-3 weeks. However, some sub-groups of patients need symptomatic or causal treatment. In addition, all patients who contact their primary-care physician with an LRTI should be informed about the severity of their disease and its prognosis.

\section{Should symptomatic acute cough be treated?}

In general, cough should be regarded as a physiological phenomenon, which is triggered by inflammation of the mucosa and helps to clear mucus from the bronchial tree. Suppression of cough is, therefore, not logical when the patient coughs up relevant quantities of sputum. However, cough can be very bothersome and tiring, especially at night. Hence, when the patient has a dry and frequent cough and nights are disturbed, suppression of cough can be useful. Dextromethorphan showed some effect in patients with acute cough, whereas studies on codeine in the same patients failed to show beneficial effects $[20,21]$. However, in patients with chronic cough both agents did diminish coughing [22].

\section{Recommendation}

Both dextromethorphan and codeine can be prescribed in patients with a dry and bothersome cough (C1).

Besides cough suppressants, there are many over-the-counter medicines available for coughing complaints. Expectorants, mucolytics and antihistamines are sold in great quantities, but consistent evidence for beneficial effects is lacking [21]. The same applies for inhaled bronchodilators in uncomplicated acute cough. To date, studies have not shown relevant beneficial effects [23].

\section{Recommendation}

Expectorant, mucolytics, antihistamines and bronchodilators should not be prescribed in acute LRTI in primary care (A1).

An important notion is that serious chronic disease, such as asthma, COPD, cardiac failure or diabetes, tends to flare up when the patient experiences an LRTI. Thus, their primary physician should consider temporarily altering the dosage of the patient's chronic medication.

\section{When should antibiotic treatment be considered in patients with LRTI?}

In the average patient with an uncomplicated LRTI in primary care, not suspected of pneumonia, antibiotic treatment has shown no benefit compared with placebo. A Cochrane review concluded that antibiotic treatment in patients with acute bronchitis had a modest beneficial effect not outweighing the side-effects of treatment. The review was hampered by the use of various outcome measures in the included randomised controlled trials (RCTs) [24, 25]. Some guidelines conclude that in LRTI where there is no suspicion of pneumonia and the diagnosis acute bronchitis should be applied, antibiotics are, therefore, not indicated. This point of view does not take into account that in subsets of patients with acute bronchitis at risk for complications, effects of antibiotics were never evaluated.
There are some indications that in the elderly, antibiotic treatment has more clinical effects than in young adults [26]. Patients with pneumonia also have an elevated risk of complications. Placebo-controlled RCTs in patients suspected of having pneumonia outside hospital are absent. However, since a large proportion of these suspected pneumonias are related to bacterial pathogens and $10-20 \%$ of these patients have a complicated disease course, it is advised to also treat these patients with an antibiotic.

\section{Recommendation}

Based on the risk for complications in certain subgroups of patients with an LRTI, antibiotic treatment is advocated in patients with an LRTI and: suspected or definite pneumonia (see How to differentiate between pneumonia and other respiratory tract infections); selected exacerbations of COPD (see What are the indications for antibiotic treatment of exacerbations of COPD?); aged $>75$ yrs and fever; cardiac failure; insulin-dependent diabetes mellitus; a serious neurological disorder (stroke etc.; C2).

What are the indications for antibiotic treatment of exacerbations of COPD?

There is considerable discussion on whether exacerbations of COPD are in fact LRTIs in patients with COPD. However, in daily practice the majority of exacerbations are treated with antibiotics. Studies on antibiotic treatment, including outpatients, show conflicting results. A meta-analysis showed a small effect on lung function [27]. The authors concluded that antibiotics might have a beneficial effect, especially in patients with a low FEV1. This conclusion was underlined by a reevaluation of these data, in which patients were stratified according to baseline lung function [28]. Another more recent meta-analysis had the same conclusions [29]. This conclusion and the fact that papers in these meta-analyses included both in- and outpatients, makes it uncertain as to whether antibiotic treatment in exacerbations in all primary care patients is useful. The two studies carried out in primary care failed to show relevant effects [30,31]. However, data on patients with severe exacerbations and exacerbations of mild and severe COPD in primary care were lacking. A key clinical trial in patients with an exacerbation of COPD showed modest beneficial effects in patients with two or more of the following three symptoms: increased sputum volume, increased sputum purulence and increased dyspnoea [32]. In conclusion, there is some evidence on beneficial effects of antibiotics in patients with severe COPD and in patients with exacerbations characterised by the above three symptoms. However, it should be noted that these criteria are subjective and based on only one study. More research in this field is needed.

\section{Recommendation}

An antibiotic should be given in exacerbations of COPD in patients with at least the following symptoms: increased dyspnoea, increased sputum volume and increased sputum purulence. In addition, antibiotics should be considered for exacerbations in patients with severe COPD (C1).

Which antibiotics should be used in patients with LRTI?

Scientifically robust, randomised, controlled, clinical trials are not available to guide this decision. S. pneumoniae, and to a 
lesser extent $H$. influenzae, are the most common bacterial pathogens in LRTI (Appendix 1). Empiric antibiotic treatment should be directed at these pathogens, even though M. pneumoniae does occur periodically. At the same time, local bacterial resistance rates should be taken into account. It is important to note that most national data on bacterial resistance rates are from microbiological laboratories where only a very small proportion of the cultures originate from primary care. Thus, these data are likely to give an overestimation of bacterial resistance outside hospital. However, it has been shown that in some European countries bacterial resistance is a relevant problem in outpatients.

Due to proven efficacy, vast experience with their use and low costs, tetracycline and amoxicillin are first-choice antibiotics, provided that locally there is no clinically relevant bacterial resistance (see Appendix 1) against both agents. Macrolides are not recommended for acute exacerbations of COPD (AECOPD) because of reduced activity against $H$. influenzae, and very high rates of pneumococcal resistance to macrolides in many European countries, but could be used for other LRTI when local bacterial resistance rates impair the effectiveness of first choice agents and in case of intolerance to these agents. Quinolones are not recommended because of concerns regarding the potential for resistance development in the community, but can be second-choice treatment in case of clinically relevant pneumococcal resistance against amoxicillin and tetracyclines, or major intolerance, such as immunoglobulin (Ig)E-mediated allergy to $\beta$-lactams. The new ketolides have little added value over macrolides and are usually more expensive. Cephalosporins also do not have a clear added value.

\section{Recommendation}

Tetracycline and amoxicillin are first-choice antibiotics. Tetracycline has the advantage that it also covers $M$. pneumoniae.

In case of hypersensitivity, a newer macrolide, such as azithromycin, roxithromycin or clarithromycin, is a good alternative in countries with low pneumococcal macrolide resistance. National/local resistance rates should be considered when choosing a particular antibiotic. When there are clinically relevant bacterial resistance rates against all firstchoice agents, treatment with levofloxacin or moxifloxacin may be considered (C4).

\section{Is anti-influenza treatment useful in patients with LRTI?}

Influenza is an important pathogen causing LRTI and is the only viral pathogen susceptible for treatment in primary care. Amantadine and rimantadine have been available for several decades. A recent meta-analysis indicated that both agents reduce the duration of symptoms on average by one day [33]. However, these drugs have been shown to induce resistance, do not work against influenza B and have frequent side-effects, mainly neurological and gastro-intestinal [34]. Since 1995, new anti-viral compounds, oseltamivir and zanamivir, have been introduced. A recent meta-analysis concluded that both agents reduced symptom duration by, on average, 0.7-1.5 days in healthy adults with a clinical flu syndrome, provided that treatment was started within $48 \mathrm{~h}$ of the onset of the clinical syndrome [35]. However, data on patients at risk for complications were very scarce. MONTO et al. [36] noticed a relative reduction of complications of influenza of $29 \%$, and a beneficial effect of treatment in patients aged $>50$ yrs ( 3 days difference). The same effect reached statistical significance in high-risk patients when patients from all available trials were pooled. However, the number of high-risk patients was limited and studies did not examine mortality as an end-point. In addition, one should realise that in the vast majority of cases, patients consult their GP far too late to benefit from anti-viral treatment.

\section{Recommendation}

The empirical use of anti-viral treatment in patients suspected of having influenza is usually not recommended (B1). Only in high-risk patients who have typical influenza symptoms (fever, muscle ache, general malaise and respiratory tract infection), for $<2$ days, and during a known influenza epidemic, can anti-viral treatment be considered (C1).

\section{How should patients with LRTI be monitored?}

There are no studies assessing what would be the best followup procedures in primary care patients with LRTI. The natural course of an uncomplicated LRTI will take 1-3 weeks.

\section{Recommendation}

A patient should be advised to return if the symptoms take $>3$ weeks to disappear.

Clinical effects of antibiotic treatment should be expected within 3 days and patients should be instructed to contact their doctor if this effect is not noticeable. Seriously ill patients, i.e. having at least two of the following symptoms/characteristics, should already be seen 2 days after the first visit: high fever; tachypnoea; dyspnoea; relevant comorbidity; aged $>65$ yrs.

All patients or persons within their environment should be advised to contact their doctor again if fever exceeds 4 days, dyspnoea gets worse, patients stop drinking or consciousness decreases (C3).

\section{MANAGEMENT INSIDE HOSPITAL Community acquired pneumonia}

Who should be admitted to hospital?

The decision to hospitalise is one of the most important steps in the management of CAP. Illness severity is the main determinant of hospital admission and this decision should be based on an objective assessment of pneumonia severity.

Two tools have been developed to guide this decision, the PSI [37] and the CURB index [38, 39].

\section{PSI}

The PSI has primarily been developed to detect those patients who can safely be treated as outpatients. According to this score, the main determinants of pneumonia severity are increasing age, comorbidity and vital sign abnormalities. However, the calculation of the PSI score requires additional laboratory, blood gas and chest radiograph data (table 6) [37].

The PSI has been firmly validated in several studies and allows the confident separation of patients with a mortality risk of up to $3 \%$ (PSI classes I-III) from those with a risk of $8 \%$ (PSI class IV) and 35\% (PSI class V) [40, 41]. 


\begin{tabular}{|c|c|}
\hline TABLE 6 Pneumonia severit & \\
\hline Criteria & Points \\
\hline \multicolumn{2}{|l|}{ Age } \\
\hline Male & Age (yrs) -0 \\
\hline Female & Age $(y r s)-10$ \\
\hline Nursing home residency & 10 \\
\hline \multicolumn{2}{|l|}{ Comorbidity } \\
\hline Neoplastic & 30 \\
\hline Liver & 20 \\
\hline Congestive heart failure & 10 \\
\hline Cerebrovascular disease & 10 \\
\hline Renal disease & 10 \\
\hline \multicolumn{2}{|l|}{ Vital sign abnormality } \\
\hline Mental confusion & 20 \\
\hline Respiratory rate $30 \cdot \mathrm{min}^{-1}$ & 20 \\
\hline Systolic blood pressure $<90 \mathrm{mmHg}$ & 20 \\
\hline Temperature $<35$ or $\geqslant 40^{\circ} \mathrm{C}$ & 15 \\
\hline Tachycardia $\geqslant 125$ bpm & 10 \\
\hline \multicolumn{2}{|l|}{ Laboratory abnormalities } \\
\hline Blood urea nitrogen $\geqslant 11 \mathrm{mmol} \cdot \mathrm{L}^{-1}$ & 20 \\
\hline Sodium $<130 \mathrm{mmol} \cdot \mathrm{L}^{-1}$ & 20 \\
\hline Glucose $\geqslant 250 \mathrm{mg} \cdot \mathrm{dL}^{-1}$ & 10 \\
\hline Haematocrit $<30 \%$ & 10 \\
\hline \multicolumn{2}{|l|}{ Radiographic abnormalities } \\
\hline Pleural effusion & 10 \\
\hline \multicolumn{2}{|l|}{ Oxygenation parameters } \\
\hline Arterial $\mathrm{pH}<7,35$ & 30 \\
\hline $\mathrm{Pa}, \mathrm{O}_{2}<60 \mathrm{mmHg}$ & 10 \\
\hline $\mathrm{Sa}_{1} \mathrm{O}_{2}<90 \%$ & 10 \\
\hline
\end{tabular}

bpm: beats per min; $\mathrm{Pa}_{1} \mathrm{O}_{2}$ : arterial oxygen tension; $\mathrm{Sa}_{1} \mathrm{O}_{2}$ : arterial oxygen saturation. The point scoring system is as follows. Risk class I: aged $<50 \mathrm{yrs}$, no comorbidity, no vital-sign abnormality; risk class II: $\leqslant 70$ points; risk class III: 71-90 points; risk class IV: 91-130 points; risk class V: $>130$ points.

The PSI has been used to validate clinical pathways. It has been found to be helpful in reducing avoidable hospital admissions, length of hospital stay and, therefore, overall costs. An impact on mortality has not been shown [42].

The PSI has three disadvantages. First, the classification of risk is a classification of the risk of mortality. This is the most important, but not the only consideration on which the decision to hospitalise should be based. The needs for supplemental oxygen or drainage of pleural effusions have been detected as main reasons to hospitalise low-risk patients [41]. Secondly, increasing age is a leading determinant within the scoring system. This may lead to an underestimation of pneumonia severity in younger patients. The third disadvantage, is the complexity of the score with the need to compute a score from 20 variables.

\section{CURB index}

The CURB index is composed of four variables (three clinical and one laboratory), which have been shown to bear important prognostic potential as part of predictive rules for pneumonia mortality in hospitalised patients (table 7) [38, 44]. These variables reflect age, acute respiratory failure and symptoms of severe sepsis or septic shock. Patients who do not meet any of

\section{TABLE 7 CURB index [43]}

\section{Four criteria assessed at admission}

Respiratory rate $\geqslant 30 \cdot \mathrm{min}^{-1}$

Diastolic blood pressure $\leqslant 60 \mathrm{mmHg}$

Blood urea nitrogen $>7 \mathrm{mmol} \cdot \mathrm{L}^{-1}$

Mental confusion

The score (0-4) is calculated by adding one point for the presence of each of the four criteria.

the four variables are at minor risk (mortality $\sim 1 \%$ ), whereas those who meet one/two or three/four are at a risk of 8 and $30 \%$, respectively.

The prognostic rules have also been validated in several studies $[38,40,45,46]$. The CURB index has been shown to allow for predictions of pneumonia mortality similar to the PSI [43]. In the latter study, age did not increase the predictive power, resulting in an even simpler score. However, the validation populations have been much smaller than those of the PSI, and the CURB index has not been validated in the ambulatory setting.

The CURB index bears two advantages as compared with the PSI. First, it is based on acute pneumonia severity and not on age and comorbidity, thereby avoiding the underestimation of pneumonia severity in younger patients or potential biases resulting from comorbidities not known or apparent at first evaluation. Secondly, it is much easier to calculate.

These objective tools should not replace subjective clinical judgment, but serve as an aid to improve the validity of clinical decision making. Moreover, nonclinical factors may justify hospitalisation in selected cases [47].

When a decision to treat as an outpatient has been made, a clinical reassessment $24-48 \mathrm{~h}$ after the first evaluation should be planned since deterioration is most likely to occur within this time [48]. Hospitalisation of patients initially treated as outpatients is not frequent and has been observed within 10 days in only about $7.5 \%$ of cases. These patients are at higher risk of death and require a longer median time to return to usual activities [49]. In case of doubt, hospitalisation should be the preferred choice. Predictors of complicated courses in seemingly low-risk patients have been identified, but not validated in independent cohorts [50].

\section{Recommendation}

The decision to hospitalise remains a clinical decision. However, this decision should be validated against at least one objective tool of risk assessment. Both the PSI and the CURB index are valid tools in this regard. In patients meeting a PSI of IV and V and/or a CURB of $\geqslant 2$, hospitalisation is recommended. Additional requirements of patient management as well as social factors not related to pneumonia severity must be considered as well (A3).

\section{Who should be considered for ICU admission?}

Severe pneumonia remains very difficult to define. Several severity criteria have been tested for their potential to predict 
ICU admission. However, this approach depends on preclinical determinants, such as the structure of local healthcare facilities. It may be impossible to derive a universally applicable prediction rule for ICU admission.

Nevertheless, the severity criteria defined so far remain useful. These criteria describe patients at increased risk of death who should receive increased attention, and ICU admission should always be considered. Criteria of increased attention may also be made in alternative settings, such as intermediate care units or even a specialised regular ward.

The potential benefits of ICU treatment should be recognised. Patients with severe pneumonia are frequently admitted to the ICU exclusively for the initiation of mechanical ventilation and/or the management of septic shock. However, due to the potential benefit of noninvasive mechanical ventilation and the documented benefit of early treatment of severe sepsis, it is not adequate to restrict ICU resources to terminal events within the ongoing inflammatory cascades in severe sepsis. Instead, careful observation of unstable patients within the ICU or intermediate care units should be applied in order to allow early and precisely targeted interventions.

Criteria for severe pneumonia include: 1) factors reflecting acute respiratory failure (e.g. respiratory rate $>30 \cdot \mathrm{min}^{-1}, \mathrm{~Pa}_{1} \mathrm{O}_{2} /$ $\mathrm{FI}_{1} \mathrm{O}_{2}$ ratio $\left.<250\right) ; 2$ ) factors reflecting severe sepsis or septic shock (e.g. hypotension (arterial systolic blood pressure $<90 \mathrm{mmHg}$, arterial diastolic blood pressure $<60 \mathrm{mmHg}$, renal failure, confusion); 3) factors reflecting amount and spread of infiltration on chest radiographs (infiltrates involving two or more lobes or bilateral infiltrates) $[48,51]$.

Although both the PSI and the CURB index provide valid estimations about the risk of the need for ICU admission, these indices have not been used to predict severe pneumonia in individual patients. Therefore, they may be only of limited value in guiding this decision. There is one predictive rule based on criteria originally proposed by the American Thoracic Society (ATS) CAP Guidelines [51] (table 8), which has been derived and validated for the prediction of ICU admission [48]. This rule is composed of three minor criteria to be assessed at admission and two major criteria which may be met at admission as well as during follow-up. It is very easy to calculate and has been shown to have a moderate positive $(75 \%)$ and excellent negative predictive value $(95 \%)$ in a Spanish setting [48]. However, probably for the reasons outlined above, it has been shown to perform less favourably in an American setting [52]. Experience with this tool is limited. The implementation of an "admission decision support" in combination with specific recommendations for outpatient treatment has been successfully used and is encouraged [51, 53, 54].

\section{Recommendation}

Criteria of acute respiratory failure, severe sepsis or septic shock and radiographic extension of infiltrates should prompt consideration of admission to the ICU or an intermediate care unit. The presence of at least two of the following indicates severe CAP and can be used to guide ICU referral: systolic blood pressure $<90 \mathrm{mmHg}$; severe respiratory failure $\left(\mathrm{Pa}_{\mathrm{a}} \mathrm{O}_{2}\right)$ $\mathrm{FI}_{1} \mathrm{O}_{2}$ ratio $<250$ ); involvement of more than two lobes in chest radiograph (multilobar involvement); or either requirement for

TABLE 8 Criteria for severe community-acquired
pneumonia according to the 2001 American
Thoracic Society Guidelines $[51]^{\#}$ (arranged
according to major and minor criteria)

mechanical ventilation or requirement of vasopressors $>4 \mathrm{~h}$ (septic shock).

The use of these facilities should not be restricted to terminal events of the course of pneumonia, but extended to all patients who are unstable in terms of the criteria mentioned (A3).

\section{What laboratory studies should be performed?}

The differentiation of mild, moderate and severe pneumonia can direct different behaviours at diagnostic work-up by laboratory and microbiological studies. All patients hospitalised with suspected CAP should receive a chest radiograph. Laboratory studies in hospitalised patients on admission should include arterial blood gas or pulse oximetry determinations [55] and basic blood chemistry (red and white blood cell count, differential cell count, creatinine and urea nitrogen, aminotransferases, sodium, potassium). CRP cannot differentiate bacterial from nonbacterial pneumonia, and is only weakly associated with severity. However, the clinical course is closely reflected by the CRP course. CRP, interleukin 6 and procalcitonin have all been shown to bear independent prognostic potential $[56,57]$. However, due to the high cost and unproven cost-effectiveness, only CRP is recommended.

Mild pneumonia does not usually require any further microbiological studies [58]. The usefulness of leukocyte counts and CRP is not proven in this group. A laboratory assessment including leukocytes and CRP, as well as a determination of blood gases, should be made in all hospitalised patients.

\section{Recommendation}

The amount of laboratory and microbiological work-up should be determined by the severity of pneumonia (A3). Microbiological work-up is primarily meant as an epidemiological investigation in order to guide future empiric 
antimicrobial policies. Occasionally, it might be helpful in guiding an individual treatment (A3).

\section{Microbiological investigation}

No study has shown that initial microbiological studies affect outcome [59]. Nevertheless, many clinicians feel that microbial investigation may be of help in guiding treatment, particularly in the more severely ill patients.

Even if a particular pathogen is detected (e.g. by bedside antigen testing), narrowing of antimicrobial spectrum cannot be generally recommended until concerns regarding the prognostic role of mixed infections have been appropriately addressed. Mixed infections have been reported to be present in $5-38 \%$ of cases $[38,60]$ and were associated with severe pneumonia in one study [61]. Moreover, several observational studies in hospitalised patients, comparing penicillin monotherapy and combination therapy of penicillin and macrolides, have raised substantial concerns into monotherapy due to adverse outcomes [62]. Thus, the role of microbiological testing is not primarily to narrow treatment options in the individual patient, but to provide more confidence about the treatment option selected in individual cases (tables 9 and 10).

What is the value of blood cultures in the diagnosis of CAP? Blood cultures have a very high specificity, but in recent studies they were positive in only $4-18 \%$ of untreated CAP cases [63-66]. They should be obtained before any antibiotic treatment and as early as possible in the disease. KALIN and LINDBERG [67] have shown that 13 out of 38 (34\%) blood cultures were positive when initiated within 4 days of the first symptoms of the illness, and three out of $26(12 \%)$ when initiated later. S. pneumoniae is identified in $\sim 60 \%$ of positive blood cultures $[68,69]$ and $H$. influenzae in various percentages from $2-13 \%$. Other organisms are recovered in diminishing order of frequency from $14 \%$ to $2 \%$ and $1 \%$ in gram negative aerobes, streptococci (Streptococcus pyogenes and other), Staphylococcus aureus and mixtures of organisms, respectively [68]. For most of the latter organisms it is difficult to decide whether they were present in the bloodstream or are skin contaminants.

\section{Recommendation}

Blood cultures should be performed in all patients with CAP who require hospitalisation (A3).

TABLE $9 \begin{aligned} & \text { Microbiological investigations in hospitalised } \\ & \text { patients with severe community-acquired } \\ & \text { pneumonia }\end{aligned}$
Microbiological investigations
Blood culture
Sputum or lower respiratory tract sample for Gram stain and culture
Pleural fluid analysis
Urinary antigen test for Legionella spp. and Streptococcus pneumoniae
Respiratory samples for direct immunofluorescence for influenza and
respiratory syncytial virus in winter months
Respiratory samples for culture or PCR for Mycoplasma pneumoniae,
Chlamydia pneumoniae and Legionella spp. if well validated test available
Initial and follow-up serology for Legionella spp. and atypical pathogens if no
PCR available; retrospective results

What other invasive techniques for normally sterile specimens can be useful in the laboratory diagnosis of pneumonia?

Thoracentesis

In $40 \%$ of CAP there may be an accompanying pleural effusion. Although specificity of pleural exudate culture is very high, the sensitivity is low because of the low incidence of invasion of the pleura [70]. Therefore, Gram stain or cultures yielding bacterial pathogens from pleural fluid are likely to be an accurate reflection of the microbial cause of the pneumonia (table 11).

\section{Recommendation}

Diagnostic thoracentesis should be performed in hospitalised patients with CAP when a significant pleural effusion is present (A3).

\section{Trans-thoracic needle aspiration}

In recent years there has been a resurgence in the interest in, and growing experience with, trans-thoracic needle aspiration (TNA) for microbial diagnosis of CAP, especially in patients with severe CAP [71-75].

TNA allows a specimen to be obtained from the infected focus without interference of commensal flora, except for possible skin contaminants. In a review of studies of patients with CAP [70], TNA has yielded a positive culture in $33-80 \%$ of cases. From 13 studies in which the results of blood cultures were also known [74], the sensitivity of lung aspiration was estimated at $74 \%$ and that of blood cultures at $37 \%$. RuIZGONZALEZ et al. [72] obtained through TNA a microbiological diagnosis in 36 out of $55(65 \%)$ patients with pneumonia of unknown aetiology by conventional methods.

The superiority of direct access to a lung lesion through TNA is also illustrated by a study that identified aetiology of infection in 12 out of $18(67 \%)$ infiltrates with a corresponding nondiagnostic BAL [76].

\section{Recommendation}

Due to the inherent potential adverse effects, TNA can only be considered on an individual basis for some severely ill patients, with a focal infiltrate in whom less invasive measures have been nondiagnostic (A3).

\section{$P S B$ and $B A L$}

The specificity of bronchoscopy for the diagnosis of bacterial pathogens in CAP is not high because of contamination with the upper airway flora. The patient may be put at unnecessary additional risk because of already-compromised gas exchange.

Several techniques have been proposed to achieve accurate discrimination between colonisation and infection. Diagnostic accuracy is improved by the use of PSB, a technique introduced by WIMBERLEY et al. [77], and BAL, at first using a bronchoscope but later not bronchoscopically taken (NB-BAL). The latter procedure is much more rapid and provides similar microbiological data in the diagnosis of ventilator-associated pneumonia [78, 79].

Quantitative bacterial culture is important for the assessment of these techniques. The cut-off point for diagnosing pneumonia has usually been set at $10^{3}$ colony forming units $(\mathrm{CFU}) / \mathrm{mL}$ by most investigators [70]. A major criticism 
TABLE 10 Diagnostic approach for the most common specific agents in lower respiratory tract infections

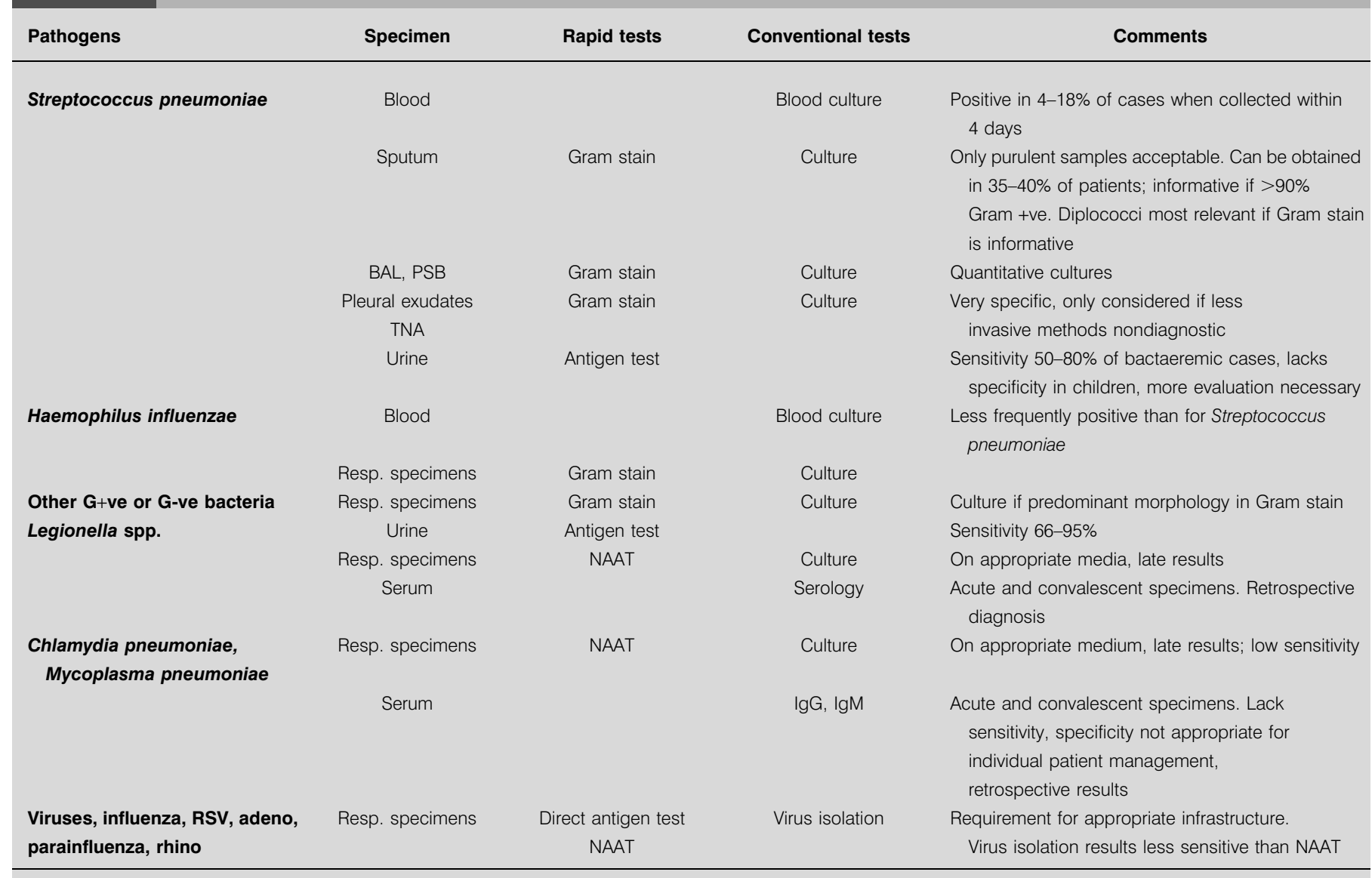

RSV: respiratory syncytial virus; BAL: bronchoalveolar lavage; PSB: protected specimen brush; TNA: total nutrient admixture; Resp.: respiratory; NAAT: nucleic acid amplification test, not generally available yet and not Food and Drug Association cleared; Ig: immunoglobulin.

\section{TABLE 11 Evaluation of a parapneumonic effusion ${ }^{\#}$}

\section{Analysis}

$\begin{array}{ll}\text { Biochemical } & \mathrm{pH}, \text { glucose, protein, } \mathrm{LDH} \\ \text { Cytological } & \text { Differential counts, exclusion of tumour cells } \\ \text { Microbiological } & \text { Stains and cultures for pyogenic bacteria, } \\ & \text { consider stains and cultures for mycobacteria }\end{array}$

LDH: lactate dehydrogenase. The process of evaluation is as follows: 1) confirm presence of exudate; 2) exclude complicated effusion or empyema, otherwise place chest drainage; 3) exclude malignancy; and 4) adjust treatment in case of identification of specific pathogen. ${ }^{*}$ : perform pleural puncture if pleural effusion exceeds $500 \mathrm{~mL}$.

directed at the PSB technique is the relatively small amount of distal bronchial secretions examined, particularly in comparison with the technique of BAL where threshold values of $\geqslant 10^{3} \mathrm{CFU} \cdot \mathrm{mL}^{-1}$ to $10^{4} \mathrm{CFU} \cdot \mathrm{mL}^{-1}$ are used $[70,80-83]$.
Using $10^{3} \mathrm{CFU} \cdot \mathrm{mL}^{-1}$ as the threshold value for a positive culture, CANTRAL et al. [84] determined the sensitivity and the specificity as $90 \%$ and $97 \%$, respectively. With a threshold value for a positive culture of $10^{4} \mathrm{CFU} \cdot \mathrm{mL}^{-1}$, the specificity of lavage cultures for potential pathogenic bacteria in relation to actual LRTI was 100\% [83]. Therefore, quantitative bacterial culture of potential pathogenic bacteria in BAL fluid is very specific, but is positive in only about one third of unselected immunocompetent adult patients with an LRTI [83].

The sensitivity of bronchoscopic BAL has been evaluated at $82-91 \%$ [85] and at $42-93 \%$ [86]. NB-BAL introduced in an emergency department allowed early identification of pathogens in severe CAP, leading to changes in antibiotic therapy [87].

\section{Recommendation}

BAL may be the preferred technique in nonresolving pneumonia (A3). Bronchoscopic sampling of the lower respiratory tract can be considered in intubated patients and selected nonintubated patients, where gas exchange status allows (A3). 
What is the value of sputum examination?

Gram strain

The most frequently submitted specimen in cases of pneumonia is sputum. To be of value for microbial diagnosis in CAP and early guide to therapy, Gram-stained sputum specimens must be representative of lower respiratory secretions and must be interpreted according to strict criteria by an experienced observer [70].

Sputum should be screened by microscopic examination for the relative number of polymorphonuclear cells and squamous epithelial cells in a low power $(100 \times)$ field. Invalid specimens $(\geqslant 10$ squamous epithelial cells and $\leqslant 25$ polymorphonuclear cells/field) should not be examined further. The cytological interobserver variability of sputum quality is satisfactory $[88,89]$.

There are great controversies regarding the value of the Gram stain. A meta-analysis of 12 studies found wide variability in sensitivity (15-100\%) and specificity (11-100\%). However, most studies showing good sensitivity and specificity used routine sputum culture in the comparison [90]. Gram-stained sputum smears can only be validated by comparing the results with those of a reference, e.g. specimens devoid of commensal flora, blood, pleural fluid cultures or TNA. There are relatively few studies in which this was carried out. Early studies [67, 91] concerned a limited number of patients, later studies involved larger numbers of patients. In a prospective study of bacteremic CAP [69], a predominant morphotype was observed in $79 \%$ of the acceptable specimens and a compatible organism was present in the blood of $85 \%$ of these patients. In another study, the conclusion was that in good-quality sputum, through the detection of a single or preponderant morphotype $( \pm 90 \%)$, the sensitivity and specificity for the detection of S. pneumoniae were 35.4 and $96.7 \%$, respectively, and for $H$. influenzae 42.8 and $99.4 \%$, respectively [92]. When a purulent sample was available the Gram stain gave a presumptive diagnosis in 175 out of 210 cases (80\%).

The main limitation is the difficulty to obtain good-quality, purulent sputum. Many pneumonia patients do not produce sputum, particularly older patients. In recent studies, satisfactory sputum specimens were obtained in: 47 out of 174 patients (32\%) [69]; 210 out of 533 patients (39\%) [92]; 23 out of 42 patients (55\%) [93]; 156 out of 205 adults (76\%) [67]; and $90 \%$ of young military recruits [94].

A low concordance of Gram-stained specimens examined by different technicians has been found [88, 89], whereas others found the results to be reproducible [95]. There is a need for laboratory quality control.

With the many limitations concerning the sputum Gram stain, is there any role for this test in the management of CAP? The answer is yes, especially if a possible pathogen is present as a predominant organism.

\section{Recommendation}

Gram stain is recommended when a purulent sputum sample can be obtained from patients with CAP and is processed timely (A3).

\section{Culture}

Sensitivity and specificity of sputum cultures are reduced by contamination with flora colonising the upper respiratory tract. The value of sputum cultures in establishing a bacterial cause of LRTI depends on how the specimens are collected and processed.

The yield of sputum cultures has varied widely, from $<20 \%$ for outpatients [58] to $>90 \%$ for hospitalised patients [91] Good concordance has been found between the results of cultures of sputum and transtracheal aspirates [94, 96], particularly when good-quality sputum specimens are washed and cultures are quantified [97].

DREW [91] detected S. pneumoniae in up to $94 \%$ (29 out of 31) of specimens from patients whose blood culture was positive. Others conclude that in cases of bacteremic pneumococcal pneumonia, S. pneumoniae may be isolated in sputum culture in only $40-50 \%$ of cases when standard microbiological techniques are used. Purulent sputa can also be obtained from patients without pulmonary pathology [98]. In some studies, the predictive value of sputum culture is low [99], even as low as $5 \%$ in cases of nonsevere CAP [58]. In one study [68] in which 19 out of $48(39.5 \%)$ of the bacteremic patients had sputum cultured, there was concordance of blood and sputum results for nine $(47 \%)$ of the pairs and in another study [99] in 25 out of $51(49.0 \%)$ of the pairs. Several approaches have been suggested to improve the sensitivity and specificity of sputum culture in CAP.

The sputum Gram stain is valuable in guiding the processing and interpretation of sputum cultures. Sputum culture results are most convincing when the organism(s) isolated in culture are compatible with the morphology of organisms present in $>90 \%$ of leukocytes in the Gram stain [70]. In the absence of an informative Gram stain, sputum cultures can only give a probable aetiological agent.

\section{Recommendation}

A culture from a purulent sputum specimen of a bacterial species compatible with the morphotype observed in the Gram stain, which is processed correctly, is worthwhile for confirmation of the species identification and antibiotic susceptibility testing (B3).

\section{What can antigen tests offer in the diagnosis of CAP?}

Rapid tests for the detection of S. pneumoniae in sputum, exudates, serum and urine have been applied over the years, most successfully counter immunoelectrophoresis, latex agglutination and enzyme immunoassays (EIA). Compared with cultures, many of these tests lack sensitivity and/or specificity or are not rapid. The latest addition is immunochromatography to be performed on urine. The test has a sensitivity of $80 \%$ in blood culture positive cases [100,101]. Urine specimens of children, carriers of S. pneumoniae in the nasopharynx, may test positive in the absence of evidence of pneumonia [102].

Antigen tests are also commonly used for the diagnosis of Legionella spp. infection. The reported sensitivity of the direct fluorescent antibody test on expectorated sputum varied from $22-75 \%$ [70]. Urinary antigen detection is currently the most helpful rapid test for the diagnosis of Legionella spp. infection. Of several test formats, the EIA format is more suited to testing 
a larger number of specimens and takes only a few hours to complete. The immunochromatographic format is better suited to single specimens and produces a result within minutes. The major limitation of urinary antigen tests is that currently available tests are intended to detect $L$. pneumophila serogroup 1 antigen, which is the most common cause of Legionella spp. infection. However, the other serogroups of L. pneumophila, or the other species of Legionella spp., are not reliably detected by this test, although cross reactions with these species do also occur [103]. These tests are particularly useful since culture of Legionella spp. is slow and takes 3-4 days. Legionella urinary antigen detection is frequently the first positive laboratory test. The sensitivity of the test is $63.7-66.6 \%$ in unconcentrated urine and $86.6-88.8 \%$ after concentration of the specimen [104] Sensitivity of the immunochromatographic assay is $55.5 \%$ and $97.2 \%$ on unconcentrated and concentrated urine specimens, respectively [105]. The assay may be negative in some patients during the first 5 days of the disease and remain positive for between 6-14 days [106].

For patients with mild Legionnaires' disease, test sensitivities range from $40-53 \%$, whereas for patients with severe Legionnaires' disease, who need immediate special medical care, the sensitivities reach $88-100 \%$ [107].

The pneumococcal urinary antigen test Binax NOW (Binax, Scarborough, ME, USA) has a sensitivity of $70-80 \%$ in adult bacteraemic pneumonia $[100,102,104]$ with a high specificity $(>95 \%)$. Its implementation in routine clinical practice and cost-effectiveness has to be evaluated further.

Antigens of the most common respiratory viruses, such as influenza, RSV, adenovirus and parainfluenza viruses, can be detected by direct immunofluorescence (DIF) or by commercially available EIA.

The sensitivities of these tests vary from $50 \%$ to $>90 \%$ [70]. Several common respiratory viruses can be detected simultaneously by the use of pooled monoclonal antibodies [108].

Half of influenza virus infections can be detected by carrying out DIF on the clinical specimen, while the other half are detected by culture on appropriate cells [109]. Dot blot immunoassays are less sensitive than DIF [109].

Especially rapid methods for the detection of influenza virus are of interest because of the availability of anti-viral agents that must be given.

\section{Recommendation}

Urine L. pneumophila serogroup 1 antigen detection is recommended for patients with severe CAP and in other patients where this infection is clinically or epidemiologically suspected (A3).

An immunochromatographic urinary antigen test for S. pneumoniae is very promising. However, its precise utility in the diagnostic microbiological strategy, which should also take cost into consideration, must await further study.

What can serological tests offer in the diagnosis of pneumonia? The serological measurement of specific antibody responses has limited application for an aetiological diagnosis of LRTI, because diagnostic results are only available retrospectively.
Efforts were made to diagnose infections caused by slowly growing or difficult-to-grow organisms by serology. This holds particularly true for M. pneumoniae, C. pneumoniae, Legionella spp. infections and viruses. The most reliable serological evidence of an ongoing infection is based on a four-fold increase in titre of $\operatorname{IgG}$ (or $\operatorname{IgG}+\operatorname{IgM}$ ) antibodies during the evolution of the disease episode based on two serum samples collected within an interval of 7-10 days, and/or the appearance of $\operatorname{IgM}$ antibodies during the evolution of the disease. IgM tests are usually less sensitive and specific than four-fold changes in antibody titres between paired specimens separated by several weeks [70]. IgM antibodies against $M$. pneumoniae require up to 1 week, and sometimes longer, to reach diagnostic titres [110]. Reported results for the sensitivity of M. pneumoniae serology are variable [111-113]. The serological responses to Chlamydia spp. and Legionella spp. take even longer [114, 115]. The acute antibody test for Legionella spp. in Legionnaires' disease is usually negative or demonstrates very low titres [116]. High titres of IgG and/or IgM above a certain threshold, present early during the disease have been interpreted as diagnostic, but at least one study showed that this titre had a positive predictive value of only $15 \%$ [116].

For M. pneumoniae a great number of antigen preparations have been proposed, e.g. whole organisms, protein fractions, glycoprotein fractions and recombinant antigens.

Some commercialised assays lack both sensitivity and specificity, emphasising the need for more validation and quality control [117].

\section{Recommendation}

Serological tests for the management of the individual patient with LRTI are not recommended (A3). Serology for infections caused by M. pneumoniae, C. pneumoniae and Legionella is more useful in epidemiological studies than in the routine management of the individual patient (A3).

\section{Are amplification tests useful for the diagnosis of LRTI?}

The newest approach in the diagnosis of respiratory tract infections is the detection of microbial nucleic acid amplification tests (NAAT), which are mostly PCR, but sometimes nucleic acid sequence based amplification (NASBA).

Many protocols have been developed mainly to detect the atypical bacteria Mycoplasma spp., Chlamydia spp., Legionella spp. and Bordetella pertussis, as well as respiratory viruses in respiratory specimens. These amplification techniques offer considerable advantages, including high sensitivity and specificity and rapid results. Furthermore, they are unaffected by prior antibiotic treatment.

NAATs are possible both in mono- and multiplex formats for the detection of one or more target organisms simultaneously [118].

The extreme sensitivity of the amplification techniques is also one of their major drawbacks as it may result in difficulties differentiating active infection from colonisation, or differentiating active from past infection. 
At present, the only amplification assay for respiratory pathogens approved by the USA Food and Drug Administration is for the diagnosis of Mycobacterium tuberculosis.

The availability of PCR for the microbiological diagnosis of LRTI is, therefore, at present generally restricted to research and reference laboratories, but is under active investigation.

Agreement between different methods is frequently low and confirmation of PCR findings by one or more alternative method has not always been performed. However, in most studies PCR-based detection of these aetiologies is more sensitive than traditional methods.

Amplification techniques, especially real-time formats, have the potential to become the ideal diagnostic tests for LRTI and may be helpful in establishing early diagnosis, allowing an aetiology-directed therapy particularly for fastidious organisms such as $M$. tuberculosis, M. pneumoniae, C. pneumoniae, Legionella spp., B. pertussis and respiratory viruses.

\section{Recommendation}

Application of molecular tests for the detection of influenza and RSV may be considered during the winter season and for the detection of atypical pathogens, provided the tests are validated and the results can be obtained sufficiently rapidly to be therapeutically relevant (A3).

\section{What classification should be used for treatment?}

Initial treatment has to be empiric. Traditional approaches for the guidance of antimicrobial treatment, such as the syndromatologic approach (typical versus atypical pneumonia), have been shown to be invalid [61, 119-122]. Instead, an approach according to the individual risk of mortality is advocated. The risk of mortality is related to pneumonia severity and allows for a distinction of: 1) low-risk patients who can safely be treated as outpatients (mild pneumonia); 2) patients at increased risk who should be hospitalised (moderate pneumonia); and 3) patients with criteria of severe pneumonia at high risk of mortality who should be admitted to the ICU (severe pneumonia).

The rapid initiation of appropriate antimicrobial treatment has been shown to be a crucial factor in order to ensure treatment success [123-125]. Therefore, any delay should be avoided. Treatment should be initiated within the first $2 \mathrm{~h}$ of hospitalisation and within the first hour after ICU admission.

It is mandatory that the initial antimicrobial regimen is appropriate since adverse patient outcomes are related to inappropriate choices [126].

In a second step, the selection of antimicrobial treatment can follow general patterns of expected pathogens according to pneumonia severity and additional risk factors $[41,60,61$, 127-132].

In hospitalised patients, S. pneumoniae has invariably been shown to represent the most frequent pathogen (Appendix 2). Any treatment regimen must ensure high activity against this microorganism. There is no uniform ranking list of the remaining potential pathogens. The relative frequency of all other pathogens is dependent on the population studied, age, regional and seasonal factors. However, $H$. influenzae,
M. pneumoniae, and C. pneumoniae are the commonest to follow [43]. M. pneumoniae tends to occur more frequently in younger people and less severe pneumonia $[43,61,133]$. The role of Legionella spp. outside of epidemics is dependent on the region studied and also varies within the same institution in different time periods [61]. Respiratory viruses play a significant role with a typical seasonal distribution pattern. Staph aureus, Gram-negative enterobacteria (GNEB) and P. aeruginosa are infrequently involved. There is a longer list of exceptional pathogens which do not need to be considered regularly unless there are risk factors hinting at the presence of one of these pathogens (table 12).

The main difference in the distribution of pathogens in severe pneumonia requiring ICU admission is the potential for a higher frequency of typical bacterial pathogens, Legionella spp., GNEB and $P$. aeruginosa $[135,136]$. Conversely, H. influenzae and $M$. pneumoniae are less frequently encountered.

The recognition of risk factors for GNEB and/or P. aeruginosa is of particular importance since it would require a different empiric initial antimicrobial treatment approach. There is considerable variability in the incidence of this pathogen across Europe. In a Spanish tertiary care and specialised setting, the presence of three out of four risk factors including COPD/bronchiectasis, recent hospitalisation, recent antimicrobial treatment, and suspected aspiration resulted in $\sim 50 \%$ risk for the presence of GNEB or P. aeruginosa [137]. Two or more of these criteria already increase the risk considerably and in high-prevalence areas should lead to consideration of antipseudomonal treatment. $P$. aeruginosa should be a concern in any patient with a smoking history and rapidly progressing pneumonia [138]. Age itself does not have a bearing on the incidence of GNEB and P. aeruginosa $[139,140]$.

Multiple pathogens have been identified in $5-38 \%$ of patients $[38,60]$, but their relevance in terms of patient outcomes has not been settled.

An issue to consider when selecting antimicrobial treatment is whether the patterns of microbial resistance are regional or local. The main concerns relate to increasing resistance of $S$. pneumoniae to penicillin and, to a lesser extent but also of note, $H$. influenzae to ampicillin. The association of microbial resistance with treatment failure has been delineated for resistance of $S$. pneumoniae to penicillin (but only above a minimal inhibitory concentration (MIC) of $2 \mu \mathrm{g} \cdot \mathrm{mL}^{-1}$ ) [141], macrolides [142, 143], and fluoroquinolones (levofloxacin) [144-146]. For penicillin resistance levels up to MIC $2 \mathrm{mg} \cdot \mathrm{dL}^{-1}$, high-dose $\beta$-lactams are still an appropriate choice $[40,41,147,148]$. The evidence for the adverse effect of resistance is best documented for macrolides.

However, the regional patterns of resistance display a huge variation, e.g. penicillin resistance reaches $50 \%$ in some regions of France and Spain, whereas it continues to be low in Germany $(\sim 5 \%)$. Therefore, treatment recommendations must be based on the consideration of local peculiarities of microbial resistance patterns. It is recommended to keep updated with the most recent information on resistance patterns as provided by resistance surveillance programmes (e.g. Alexander project [149], SENTRY [150] and European Anitmicrobial Resistance Surveillance System (EARSS; http://www.earss.rivm.nl/). 


\begin{tabular}{|c|c|c|}
\hline TABLE 12 & \multicolumn{2}{|c|}{$\begin{array}{l}\text { Additional risk factors to consider when } \\
\text { selecting empiric initial antimicrobial treatment }\end{array}$} \\
\hline Risk factor & & Pathogen \\
\hline \multicolumn{2}{|c|}{ COPD and/or bronchiectasis } & $\begin{array}{l}\text { Haemophilus influenzae, GNEB, } \\
\text { Pseudomonas aeruginosa }\end{array}$ \\
\hline \multicolumn{2}{|c|}{ Recent hospitalisation } & GNEB, Pseudomonas aeruginosa \\
\hline \multicolumn{2}{|c|}{ Recent antimicrobial treatment } & GNEB, Pseudomonas aeruginosa \\
\hline \multicolumn{2}{|c|}{ Silent aspiration } & Mixed infections, anaerobes \\
\hline \multicolumn{2}{|c|}{ Gross aspiration } & $\begin{array}{l}\text { GNEB, Pseudomonas aeruginosa, } \\
\text { anaerobes }\end{array}$ \\
\hline \multicolumn{2}{|l|}{ Influenza } & $\begin{array}{l}\text { Staphylococcus aureus, Streptococcus } \\
\text { pneumoniae, Haemophilus influenzae }\end{array}$ \\
\hline \multicolumn{2}{|c|}{ Exposure to cattle } & Coxiella burnetii \\
\hline \multicolumn{2}{|c|}{ Exposure to birds } & Chlamydia psittaci \\
\hline \multicolumn{2}{|c|}{ IVDA } & Staphylococcus aureus (MSSA or MRSA) \\
\hline \multicolumn{2}{|c|}{$\begin{array}{l}\text { Recent travel to Mediterranean } \\
\text { coast }\end{array}$} & Legionella spp. \\
\hline \multicolumn{2}{|c|}{$\begin{array}{l}\text { Recent travel to Midwest and } \\
\text { Southern USA }\end{array}$} & Histoplasma capsulatum \\
\hline \multicolumn{2}{|c|}{ Chronic steroid treatment } & Aspergillus spp. \\
\hline
\end{tabular}

COPD: chronic obstructive pulmonary disease; GNEB: Gram-negative enterobacteria; IVDA: intravenous drug abuse; MSSA: methicillin-sensitive Staphylococcus aureus; MRSA: methicillin-resistant Staphylococcus aureus. \#: the presence of two or more of COPD/bronchiectasis, recent hospitalisation, recent antimicrobial treatment, and suspected aspiration, resulted in increased risk for the presence of GNEB or Pseudomonas aeruginosa [134]

Since the individual risk of resistance has been shown to be related to antibiotic use prior to admission, the report of previous use of a specific antimicrobial agent should be considered when selecting empiric treatment [151].

Finally, considerations of tolerability and toxicity of antimicrobial agents in the individual patient have to take place. The main issues relate to patients with significant comorbidities and to elderly patients. The most important of these are listed in table 13.

\section{Recommendation}

Antimicrobial treatment has to be empiric and should follow an approach according to the individual risk of mortality. The assessment of severity according to mild, moderate and severe pneumonia implies a decision regarding the most appropriate treatment setting (ambulatory, hospital ward, ICU; A4). Antimicrobial treatment should be initiated as soon as possible (A3).

The guidance of empiric initial antimicrobial treatment should follow: 1) general patterns of expected pathogens according to pneumonia severity and additional risk factors; 2 ) regional and local patterns of microbial resistance; 3) considerations of tolerability and toxicity of antimicrobial agents in the individual patient.

The implementation of a clinical pathway to ensure appropriate care for patients with CAP is encouraged. This pathway should be focussed on the critical issues associated with patient outcomes. Benefits in terms of morbidity and costs
TABLE 13 Risk factors for toxicity and poor tolerance of antimicrobial agents

\begin{tabular}{lcc} 
Antimicrobial agents & Toxicity & Risk factor \\
\hline $\begin{array}{l}\boldsymbol{\beta} \text {-lactams } \\
\text { Macrolides }\end{array}$ & Allergy & History of penicillin allergy \\
& Ototoxicity & Age \\
Fluoroquinolones & Arrhythmias & Prolonged QT \\
& Arrhythmias & Heart failure, electrolyte \\
& CNS & imbalances, prolonged QT \\
& Tendinitis & CNS disorders \\
Aminoglycosides & Nephrotoxicity & Concomitant steroids \\
& Ototoxicity & Agencomitant diuretics \\
\hline
\end{tabular}

CNS: central nervous system; QT: interval on electrocardiogram.

have been demonstrated [42, 152-154]. A reduction in mortality has not been proven $[42,155]$.

\section{What initial empiric treatments are recommended?}

In general, all comparative studies are based on a design testing for equivalence. Therefore, it remains uncertain whether, and to what extent, antimicrobial and pharmacokinetic advantages of distinct antimicrobial agents result in better treatment outcomes. Moreover, most studies have not included patients with severe pneumonia or criteria of severity.

Interesting data have been provided in large retrospective series investigating different antibiotic treatment regimens, e.g. three regimens were found to be associated with a lower 30 day mortality in a large cohort of elderly patients. These were: second-generation cephalosporin plus macrolide; thirdgeneration nonpseudomonal cephalosporin plus macrolide; and fluoroquinolone alone [156]. Other authors found monotherapy with third-generation cephalosporin to be equally effective as compared with a combination treatment with a macrolide [157]. However, these data are retrospective and hypothesis-generating rather than confirmative.

In conclusion, data to support the use of different antimicrobial agents in these patients remains very limited. The general options of antimicrobial treatment are given in tables 14 and 15. Patients hospitalised for reasons other than pneumonia severity (i.e. with a risk such as outpatients) may accordingly be treated like outpatients.

Criteria for the addition of a macrolide in patients with moderate pneumonia have not been settled. Clinical criteria for atypical pathogens are definitely invalid to guide this decision. Moreover, the relevance of atypical pathogens remains a subject of controversy [158]. Conflicting data have been published for the treatment of pneumococcal pneumonia. Whereas some authors safely used procaine penicillin monotherapy [159], others found a combination of penicillin and macrolide to be associated with lower mortality as compared with $\beta$-lactam monotherapy [62]. The current authors advocate the addition of macrolides, at least in patients at increased risk and in patients presenting with criteria of severe pneumonia [160]. 


\begin{tabular}{|c|c|}
\hline TABLE 14 & $\begin{array}{l}\text { Recommended treatment options (in no special } \\
\text { order) for hospitalised patients with community- } \\
\text { acquired pneumonia (C4) }\end{array}$ \\
\hline Preferred & Alternative $^{+}$ \\
\hline \multicolumn{2}{|c|}{ 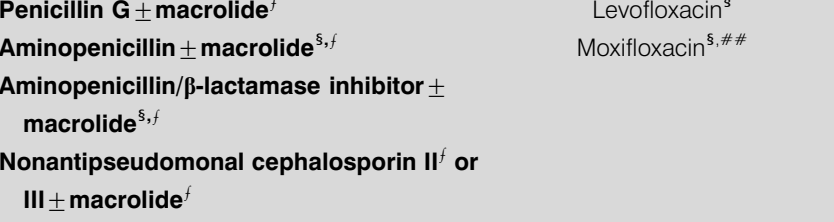 } \\
\hline \multicolumn{2}{|c|}{ 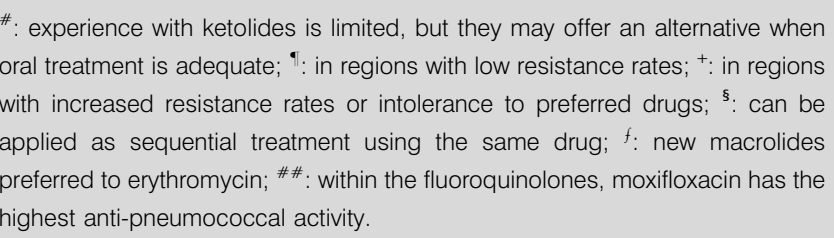 } \\
\hline
\end{tabular}

\section{TABLE 15}

Recommended treatment options for patients with severe community-acquired pneumonia (C4)

\section{No risk factors for $P$. aeruginosa}

Nonantipseudomonal cephalosporin III + macrolide or Nonantipseudomonal cephalosporin III + (moxifloxacin or Levofloxacin)

\section{Risk factors for $\boldsymbol{P}$. aeruginosa}

(Anti-pseudomonal cephalosporin or

Acylureidopenicillin/ $\beta$-lactamase inhibitor or

Carbapenem) + ciprofloxacin ${ }^{\#}$

P. aeruginosa: Pseudomonas aeruginosa. \# : evidence in favour of combination therapy for $P$. aeruginosa remains inconclusive. Aminoglycoside therapy is associated with increased toxicity and monotherapy often leads to the development of resistance against that antibiotic. Therefore, combination therapy with a $\beta$-lactam plus a fluoroquinolone is advocated.
A particular ranking in recommendations is explicitly not given. It is important to realise that the exclusive use of single antimicrobial options may carry the risk of inducing microbial resistance.

A very important issue is adequate dosing. New concepts of dosage calculation by use of pharmacokinetic and pharmacodynamic data are promising, but still controversial and hardly applicable in a clinical routine [161]. Recommended dosages are summarised in Appendix 3. Dosage has to be adjusted according to the presence of liver or renal failure.

\section{What is the recommended treatment for specific identified pathogens?}

Adjusted treatment may be necessary in case of the identification of a specific pathogen. Possible choices are listed in table 16

\section{What should be the duration of treatment?}

The appropriate duration of antimicrobial treatment has not been settled. In comparative studies, the usual duration of treatment is around 7-10 days. However, whether shorter durations can be safely applied is unknown. Intracellular pathogens such as Legionella spp. should be treated for at least 14 days (C4).

\section{When should i.v. be used and when should the switch to oral} occur?

In hospitalised patients not requiring ICU admission, current evidence supports the use of sequential treatment unless there are contraindications, such as impaired gastrointestinal absorption [162-165]. The safety of switching has also been confirmed for bacteremic pneumococcal pneumonia [166]. In low-risk patients hospitalised for reasons other than increased risk of mortality according to PSI or CURB index, oral treatment may be applied from the beginning. In addition, patients at increased risk, but without vital sign abnormalities may be candidates for oral treatment from the beginning [167, 168]. Since the clinical benefit of in-hospital observation in patients at low risk after switching to oral treatment remains limited $[165,169]$, the decision to switch should be accompanied by a consideration of discharge. Early switch and

TABLE 16 Recommended treatment for specific identified pathogens

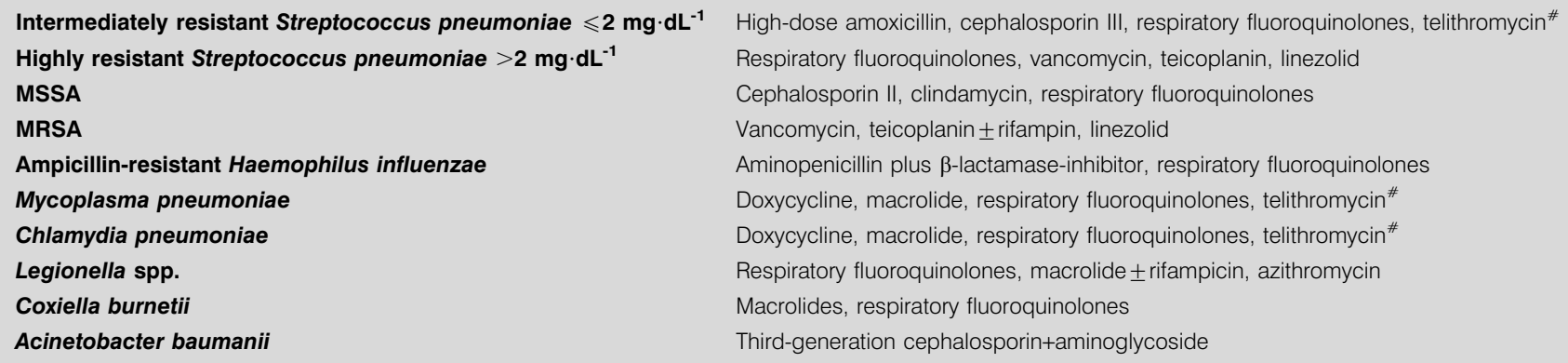

High-dose amoxicillin, cephalosporin III, respiratory fluoroquinolones, telithromycin ${ }^{\#}$ Respiratory fluoroquinolones, vancomycin, teicoplanin, linezolid Cephalosporin II, clindamycin, respiratory fluoroquinolones

Vancomycin, teicoplanin \pm rifampin, linezolid Aminopenicillin plus $\beta$-lactamase-inhibitor, respiratory fluoroquinolones Doxycycline, macrolide, respiratory fluoroquinolones, telithromycin ${ }^{\#}$ Doxycycline, macrolide, respiratory fluoroquinolones, telithromycin ${ }^{\#}$ Respiratory fluoroquinolones, macrolide \pm rifampicin, azithromycin Macrolides, respiratory fluoroquinolones Third-generation cephalosporin+aminoglycoside

MSSA: methicillin-sensitive Staphylococcus aureus; MRSA: methicicllin-resistant Staphylococcus aureus. ${ }^{\#}$ : experience with ketolides is limited, but they may offer an alternative when oral treatment is adequate. 
discharge strategies may help to reduce the length of hospitalisation $[163,170]$. There are only limited data regarding the safety of sequential treatment in severe pneumonia [168].

Favourable clinical responses have been reported for several sequentially applied antimicrobial drug regimens. In theory, the most appropriate are drugs which are available both for i.v. and oral use and which achieve the same area under the curve (AUC) regardless of application route [171, 172]. However, a sequential treatment regimen using oral drugs with lower AUC has been successfully applied. Table 17 gives an overview of possible choices of sequential treatment in CAP.

The time to switch from i.v. to oral antimicrobial treatment has to be individualised according to clinical features of stabilisation. One study used stable resolution of fever (two consecutive normal body temperatures within $8 \mathrm{~h}$ ), improvement of cough and decrease of white blood cells [163]. Another treated patients at least $48 \mathrm{~h}$ intravenously before switching to oral treatment [164]. Probably the most practical approach is the use of the most prominent clinical features at admission (e.g. fever, acute respiratory failure or confusion) as target in the assessment of clinical stabilisation. The practice of observing the patient overnight after change to oral treatment is common, but has not been proved necessary [165].

\section{Recommendation}

In mild pneumonia, treatment can be applied orally from the beginning (A3). In patients with moderate pneumonia, sequential treatment should be considered in all patients except the most severely ill. The optimal time to switch to oral treatment is also unknown. It seems reasonable to target this decision according to the resolution of the most prominent clinical features at admission (A3).

\section{Which additional therapies are recommended?}

Prophylactic treatment with low molecular heparin $(40 \mathrm{mg}$ enoxaparin s.c.) has been shown to protect patients suffering from acute respiratory failure. It reduced thromboembolic

\begin{tabular}{|c|c|c|}
\hline $\begin{array}{l}\text { Types a } \\
\text { antimicr }\end{array}$ & $\begin{array}{l}\text { d possible choices } \\
\text { bial treatment }\end{array}$ & sequential \\
\hline $\begin{array}{l}\text { Sequential } \\
\text { treatment regimen }\end{array}$ & Intravenous drug & Oral drug \\
\hline \multirow[t]{4}{*}{ Same drug/identical AUC } & Moxifloxacin & Moxifloxacin \\
\hline & Levofloxacin & Levofloxacin \\
\hline & Ciprofloxacin & Ciprofloxacin \\
\hline & Clindamycin & Clindamycin \\
\hline \multirow[t]{5}{*}{ Same drug/lower AUC } & $\begin{array}{c}\text { Aminopenicillin } \\
\text { (Ampicillin/amoxicillin) }\end{array}$ & Amoxicillin \\
\hline & Cufuroxime & Cefuroxime-axetil \\
\hline & Erythromycin & Erythromycin \\
\hline & Clarithromycin & Clarithromycin \\
\hline & Azithromycin & Azithromycin \\
\hline \multirow{2}{*}{$\begin{array}{l}\text { Different drugs/different } \\
\text { AUC }\end{array}$} & Ceftriaxone & Cefuroxime axetil \\
\hline & & Cefixime \\
\hline
\end{tabular}

events from $14.9 \%$ to $5.5 \%$ and occasionally prevented embolic complications [173]. Therefore, low molecular heparin should be applied to all patients with acute respiratory failure.

The use of noninvasive ventilation has been shown to be effective in COPD patients with pneumonia [174]. Its use in patients with comorbidities, other than COPD, remains controversial [175]. The principles to be applied when using noninvasive ventilation have been addressed elsewhere [176].

Patients with severe sepsis should receive adequate fluid resuscitation. It is mandatory to institute timely appropriate supportive treatment to all patients with septic shock.

Steroids have not been shown to be useful in pneumonia treatment. However, hydrocortisone has been shown to be beneficial in patients with septic shock. Granulocytemacrophage colony-stimulating factor has been shown to reduce acute renal failure, but without any impact on mortality [177]. Therefore, it is not recommended for treatment of severe CAP.

\section{Recommendation}

Low molecular heparin is indicated in patients with acute respiratory failure (A3). The use of noninvasive ventilation is not yet standard of care, but may be considered particularly in patients with COPD (B3). The treatment of severe sepsis and septic shock is confined to supportive measures (A3). Steroids have no place in the treatment of pneumonia unless septic shock is present (A3).

\section{How should responses be assessed and when should chest radiographs be reported?}

The first 24-72 $\mathrm{h}$ after admission are crucial for the development of pneumonia. However, late complications may occur. These are usually related to the development of empyema, nosocomial superinfections or complications of comorbidities [137]. The time required for complete resolution of symptoms and signs of pneumonia may reach much longer periods [178]. It depends on both host- and pathogen-related factors as well as the initial severity of pneumonia. Therefore, it seems practical to differentiate initial response and complete resolution, particularly when assessing treatment response.

Monitoring of the patient should include: body temperature; parameters of respiration (preferably respiratory rate and partial oxygen tension or oxygen saturation); haemodynamics (arterial blood pressure and heart rate); and mental state. Early referral to an intermediate care unit or the ICU may be helpful in patients with unfavourable evolution according to these criteria.

The chest radiograph alone is not appropriate to assess initial treatment response. However, in the context of a worsening clinical condition, an increase of infiltrates of more than half compared with the initial findings is indicative of a poor prognosis [48, 135].

The radiographic confirmation of uncomplicated pneumonia resolution is common clinical practice. However, the time required for complete radiographic resolution is dependent on host and pathogen factors and usually longer than expected $[179,180]$. It can probably be confined to patients with persistent symptoms and signs, as well as those at risk of 
bronchial neoplasm (i.e. present smokers and ex-smokers). In patients with severe pneumonia, repeated chest radiographs are part of patient management and can be performed according to clinical judgement.

The time frame for stabilisation depends on the criteria for stabilisation applied and initial pneumonia severity [181]. Following several criteria defined below, patients with PSI IV and $\mathrm{V}$ display median stabilisation times of 3 and 5 days, respectively [182]. The relevant criteria include: defervescence $<37.9^{\circ} \mathrm{C}$; respiratory rate $<25 \cdot \mathrm{min}^{-1}$; oxygen saturation $>89 \%$; arterial systolic pressure $>90 \mathrm{mmHg}$; heart rate $<100 \cdot \mathrm{min}^{-1}$; ability to maintain oral intake; and normal mental status.

If all criteria are met, the risk for readmission or death after discharge is $\sim 14 \%$. Conversely, the presence of two or more vital sign abnormalities at discharge carries a $46 \%$ risk of readmission or death. Therefore, all the criteria listed above should be considered prior to the decision regarding hospital discharge.

Duration of hospitalisation is one of the most important cost drivers in healthcare for CAP. Reduction of hospitalisation time by even 1 day can yield substantial cost-savings [183]. Therefore, it seems reasonable to target treatment durations according to predicted hospitalisation times. The predicted duration of hospitalisation has been reported to reach $\sim 5$ days for patients at lower risk (PSI I-III) and 7 days for patients at increased risk (PSI IV and V) [37, 184]. However, substantial regional and national variations have to be taken into account. Prolonged hospitalisation times depend on pneumonia-related factors (initial severity, early complications), nonpneumoniarelated complications and nonclinical factors [184].

There is evidence that the duration of hospitalisation can safely be reduced in many institutions [185]. However, discharge decisions should be based on confident markers of clinical stabilisation. Otherwise, there is concern that shortening of hospitalisation may result in a higher proportion of adverse outcomes [182, 186]. Tools for a safe reduction of hospitalisation times include: 1) early switch to oral antimicrobial treatment, a practice guideline providing information about switching patients from parenteral to oral antimicrobial regimen may be useful for early discharge of patients [182, 187]; and 2) interventions to reduce the impact of nonclinical factors.

\section{Recommendation}

Response to treatment should be monitored by simple clinical criteria including body temperature, respiratory and haemodynamic parameters. The same parameters should be applied to judge the ability of hospital discharge (A3). Complete response, including radiographical resolution, requires longer time periods. Discharge decisions should be based on robust markers of clinical stabilisation (A3).

\section{How should the nonresponding patient be assessed?}

It seems practical to differentiate failure to respond to initial treatment (addressed as nonresolving pneumonia) and failure of complete resolution (addressed as slowly resolving pneumonia). Prior to labelling a patient as a nonresponder, it should be made sure that: 1) the patient is most likely to have CAP (i.e. correctly classified on clinical grounds); 2) the antimicrobial treatment applied was correctly selected (judging choice of agent, route of administration, dosage); 3) if specific pathogens were identified, these were treated appropriately.

Having confirmed the diagnosis of treatment failure, the next step is to assign the patient to one of the two categories of treatment failure.

\section{Nonresolving pneumonia}

If the patient is unstable (progressive pneumonia), all efforts should be made to reinvestigate the patient intensively. The use of particular diagnostic tools, e.g. computed tomography (CT) scanning or bronchoscopy must be individualised [137]. In case of using bronchoscopy, it must be made sure that the retrieved samples are comprehensively investigated. The most important diagnoses to exclude are empyema, abscess formation, PE and fluid overload. After collecting all samples for microbiology analysis, a second empiric antimicrobial treatment regimen using different agents than initially applied should be initiated.

If the patient is stable, the extent of re-investigation can be individualised. A second antimicrobial treatment course may be temporarily withheld until a differential diagnosis is made.

\section{Slowly resolving pneumonia}

The decision to embark on re-investigation should be based on a careful estimation of factors influencing symptom resolution and the likelihood of identifying a specific diagnosis. This type of treatment failure makes it more likely to find unusual pathogens or alternative diagnoses mimicking pneumonia, such as interstitial lung disease or malignancy. Therefore, the clinical and radiographic presentation may hint at the presence of one of these conditions. CT scanning may be helpful in case of suspected interstitial lung disease. Bronchoscopic evaluation of the patient will be rewarding in up to half of the cases [137, 188]. The potential usefulness of open lung biopsy cannot be judged outside of a specific individual condition.

\section{Recommendation}

Two types of treatment failures, nonresponding pneumonia and slowly resolving pneumonia, should be differentiated (A3). The evaluation of nonresponding pneumonia depends on the clinical condition. In unstable patients, full re-investigation followed by a second empiric antimicrobial treatment regimen is recommended. The latter may be withheld in stable patients. Slowly resolving pneumonia should be reinvestigated according to clinical needs, condition of the patient and individual risk factors (C3).

\section{Exacerbations of COPD}

What are the criteria for hospital admission?

The mortality of patients hospitalised with COPD exacerbation is $\sim 10 \%$. It is important for clinicians to have sensitive and specific criteria for the hospitalisation of these patients. The specific criteria for hospitalisation is still a matter of debate, but as a general rule all patients with a COPD exacerbation that will not show improvement after $12 \mathrm{~h}$ of development, with persistence of respiratory failure and intense dyspnoea, should be admitted.

The new ERS/ATS statement has made recommendations on specific criteria to hospitalise patients with COPD exacerbation [189]. As yet, these criteria have not been validated (table 18). 


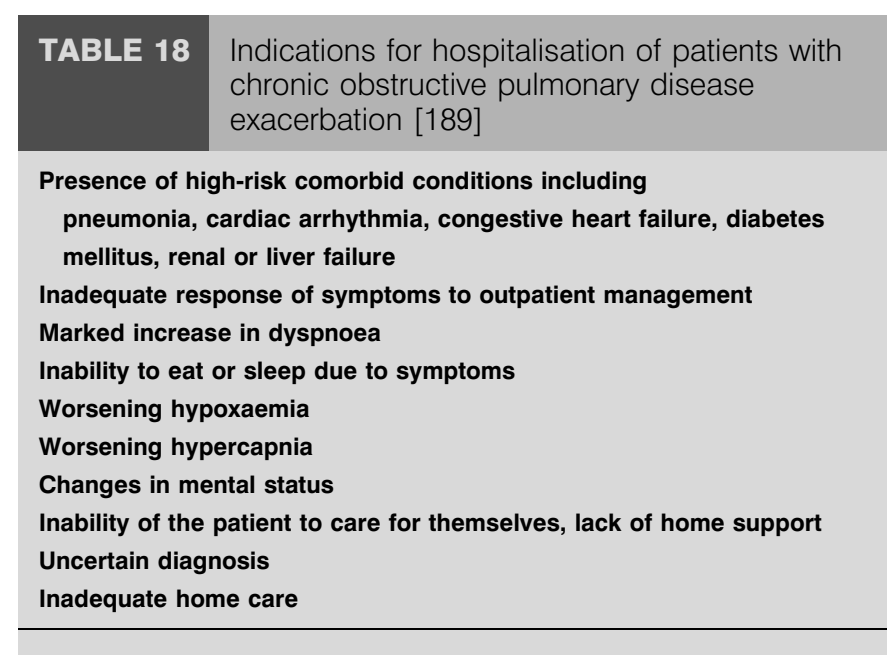

It is interesting for clinicians to know the risk factors associated with admission in patients with a COPD exacerbation. A case control study on risk factors for COPD exacerbation and hospital admission found that the following were associated with admission in a multivariate analysis [190]: three or more hospital admissions in the previous year; FEV1; under prescription of long-term oxygen therapy; and being a current smoker.

In the process for hospital evaluation it is important as a first step to know and stratify the severity of the disease. This is determined by the degree of functional impairment in the stable condition; forced spirometry is the best tool for this. In addition to previous lung function, signs and symptoms of acute morbidity should also be used to stratify patients according to severity (severity of hypoxaemia, hypercapnia with acidosis, tachypnoea and others).

The new ERS/ATS statement has proposed a classification to stratify COPD severity according to the degree of airflow obstruction, i.e. mild COPD: FEV1 $\geqslant 80 \%$; moderate COPD: FEV1 50-80\%; severe COPD: FEV1 30-50\%; very severe COPD: FEV1 $<30 \%$.

\section{Which hospitalised patients with COPD exacerbations should receive antibiotics?}

In clinical practice clinicians should be able to detect which patients do not need to receive antibiotics and those in whom antibiotics will be potentially useful. Not all COPD patients presenting with an exacerbation need to be treated with antibiotics. One meta-analysis [27] examining randomised, placebo-controlled trials showed an overall modest beneficial effect of the administration of antibiotics for COPD exacerbations. This meta-analysis was published some years ago and most of the antibiotics included in the trials evaluated were less potent compared with the antibiotics which are currently available.

The cornerstone randomised trial by ANTHONISEN et al. [32] showed a significant beneficial effect of antibiotics in those patients who presented with all three of the following symptoms: increase in dyspnoea; increase in sputum volume; and increase in sputum purulence. These patients were classified as Type I. In addition, the administration of antibiotics was beneficial in those patients with two cardinal symptoms, this was classified as Type II. Figure 1 shows the stratification of exacerbated patients according to ANTHONISEN et al. [32]. Recent information in nonhospitalised exacerbated patients shows a clear relationship between the purulence of sputum and the presence of bacteria [15]. The presence of green sputum (using a colour scale) was $94 \%$ sensitive and $77 \%$ specific for the yield of a high bacterial load, indicating a clear subset of patient episodes identified at presentation that are likely to benefit most from antibiotic therapy. This information complements the study of ANTHONISEN et al. [32] in the sense that patients having two of the cardinal symptoms (Type II), but including the presence of purulence (green sputum) should be treated with antibiotics. However, the study of ANTHONISEN et al. [32] was performed in ambulatory patients and patients who needed later admission after inclusion in the study and were excluded from the analysis. The use of the Anthonisen criterion has not been validated in hospitalised COPD exacerbated patients.

Two randomised studies have clarified which patients should or should not receive antibiotics. One of them studying ambulatory patients with mild symptoms (simple chronic bronchitis) showed no beneficial effects of antibiotics [25]. The other investigated COPD patients requiring mechanical ventilation and showed that not administering antibiotics was followed by an adverse outcome and a greater incidence of secondary intra-hospital infections [191]. There are no available data on which patients with COPD exacerbations receiving noninvasive mechanical ventilation could benefit from antibiotics, but in the clinical practice they should be regarded as mechanically ventilated.

Most COPD patients requiring hospitalisation have severe disease $(\mathrm{FEV} 1<50 \%)$ and have a Type I or II exacerbation according to the Anthonisen classification [192].

\section{Recommendation}

Antibiotics should be given to the following: 1) patients with a Type I Anthonisen exacerbation (A2); 2) patients with a Type II Anthonisen exacerbation when increased purulence of sputum is one of the two cardinal symptoms (A2); and 3) patients with a severe exacerbation that requires invasive or noninvasive mechanical ventilation (A2). Antibiotics are generally not recommended in Anthonisen Type II without purulence and Type III patients (A2).

\section{What stratification of patients with COPD exacerbation is recommended to direct treatment?}

Exacerbations of COPD may be the result of a viral infection, a bacterial infection or irritants including tobacco smoke and others. The role of bacterial infection is questioned but bronchoscopic studies have shown that at least $50 \%$ of patients may have bacteria in high concentrations in their lower airways during exacerbations [193]. The problem in defining the microbial aetiology of a COPD exacerbation is that a great proportion of these patients have bacteria colonising their lower airways in the stable phase of the disease. Some studies suggest that the bacterial burden increases during exacerbations [193] and different strains of the same bacteria may change when comparing exacerbations in the same patients [194]. The latter study found that strain changes for 


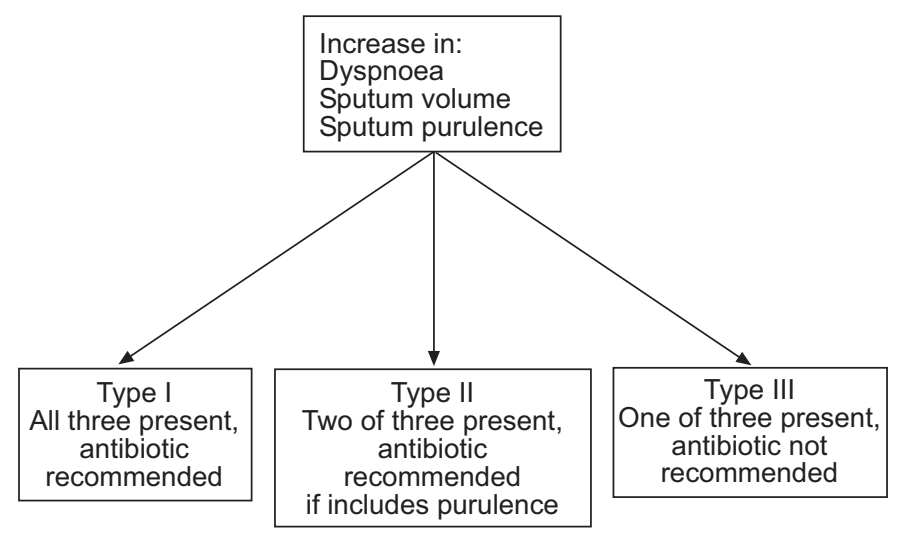

FIGURE 1. Patient stratification by characteristics of exacerbation.

H. influenzae, S. pneumoniae and Moraxella catarrhalis, but not for $P$. aeruginosa, were associated with exacerbation.

Studies in patients with mild exacerbations [195], which do not require hospital admission, have shown that half of these patients have bacteria in their lower airways and that the predominant microorganisms are $H$. influenzae (nontypable and noncapsulated), S. pneumoniae and $M$. catarrhalis. In contrast, in patients requiring mechanical ventilation (mean FEV1 30\%) [196, 197] the role of these microorganisms is less frequent and other microorganisms, such as enteric Gram negative bacilli and $P$. aeruginosa may be more frequent. Other observations have shown that FEV1 is an important determinant of the type of microorganism [198, 199]. In patients with mild disease $S$. pneumoniae is predominant, whereas when FEV1 is lower, $H$. influenzae and $M$. catarrhalis are more frequent and even $P$. aeruginosa may appear in those patients with more severe obstruction. In patients with severe COPD and frequent exacerbations requiring antibiotics and steroids the microbial aetiology is more complex and enteric Gram negative bacilli and $P$. aeruginosa may be present [200].

The definition of risk factors for $P$. aeruginosa infection is a potentially important issue. The antibiotic treatment is totally different compared with the treatments directed to common microorganisms and the evolution of the patients can be worse if anti-pseudomonal antibiotics are not administered very early [196]. The risk factors for $P$. aeruginosa infection are the following: 1) recent hospitalisation; 2) frequent administration of antibiotics (four courses in the last year); 3) severe COPD (FEV1 $<30 \%$ ); and 4 ) isolation of $P$. aeruginosa during a previous exacerbation or colonisation during a stable period [198, 199]. However, most of these factors have been found in general studies investigating the microbiology of the exacerbation and specific studies on risk factors for $P$. aeruginosa in exacerbations are lacking. Overall, the percentage of Pseudomonas infection is $\sim 10-15 \%$ in patients with COPD exacerbations requiring hospitalisation and who have FEV1 $<50 \%$. This percentage is increased in patients admitted to ICU needing mechanical ventilation. However, the issue of $P$. aeruginosa in COPD exacerbations is far from clear and no study has investigated whether specific treatment for $P$. aeruginosa, on the basis of risk factors, alters outcome.
The role of atypical bacteria is controversial, but most studies show that C. pneumoniae is found in $\sim 4-20 \%$ of exacerbations [200], with M. pneumoniae being less frequent. In one of these studies, C. pneumoniae had a $4 \%$ incidence when considering hospitalised COPD patients [201]. C. pneumoniae is frequently found in association with other viral or bacterial microorganisms. Viruses are also frequent microorganisms found in COPD exacerbations. The range of incidence is highly variable depending on the type of microbiological techniques used and the type of viruses searched for. One study from SEEMUNGAL et al. [202] found an incidence of viral infection of $39 \%$ with rhinovirus and RSV the most frequent. Other frequent viruses that are potentially involved are picornaviruses and influenza A.

The pattern of antibiotic resistance of microorganisms usually identified in exacerbations is country specific [203]. In Spain, France and Italy up to $40 \%$ of strains of $H$. influenzae and $>90 \%$ of $M$. catarrhalis are $\beta$-lactamase producers, which suggests that antibiotics such as ampicilin and amoxicillin may be ineffective. S. pneumoniae is, in addition, frequently resistant to penicillin and macrolides, although this also importantly varies from country to country (see Appendix 2, Antibiotic resistance section).

Finally, $10-20 \%$ of patients with moderate-severe exacerbations don't respond to initial antibiotic treatment and may require a change in the antibiotic treatment [204, 205]. In these cases the infection may be caused by $P$. aeruginosa and Staphylococcus aureus, microorganisms that are usually not well covered by the standard empirical regimes.

Stratification of patients for antibiotic administration helps to define groups of patients in whom particular microorganisms may be potentially present. This is a difficult issue that should combine host variables (risk factors for resistances to $S$. pneumoniae, penicillin and macrolides and risk factors for $H$. influenzae $\beta$-lactamase producer), risk factors for $P$. aeruginosa and signs and symptoms of acute morbidity that could define subsets of populations needing particular antibiotic treatment. The use of FEV1 as a variable to stratify patients for antibiotic treatment is not always useful in the clinical practice since this information may not be available at emergency department attendance. However, when FEV1 is available it helps to classify patients into different groups for antibiotic treatment.

\section{Recommendation}

There are three different groups. Group A comprises patients not requiring hospitalisation (mild COPD, see Prevention section; A3). Group B is patients that are admitted to hospital (moderate-severe COPD) without risk factors for P. aeruginosa infection (A3). Finally, Group C comprises patients admitted to hospital (moderate-severe COPD) with risk factors for P. aeruginosa (A3).

\section{What are the risk factors for $P$. aeruginosa}

The risk factors for $P$. aeruginosa are two of the following: 1) recent hospitalisation (A3); 2) frequent (more than four courses per year) or recent administration of antibiotics (last 3 months; $\mathrm{A} 3)$; 3) severe disease (FEV1 $<30 \%$; A3); 4) previous isolation of $P$. aeruginosa during an exacerbation or patient colonised by $P$. aeruginosa (A3). The stratification of patients with COPD is shown in table 19. 


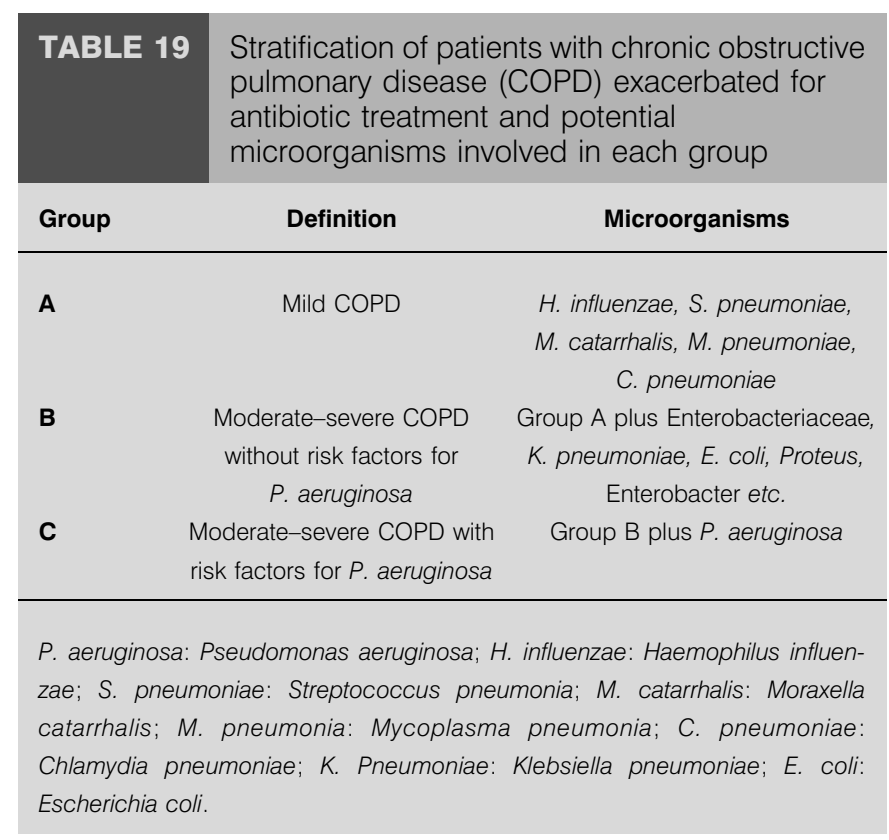

\section{Which microbiological investigations are recommended?}

Commensal pharyngeal flora may interfere with microbiological investigations. Sampling of distal airways by bronchoscopy and collection of PSB revealed that some COPD and bronchiectasis patients are chronically colonised by bacteria during stable periods, but with bacterial numbers increasing during exacerbations.

Studies of PSB in stable COPD and bronchiectasis patients found a prevalence of $10^{3}-10^{4} \mathrm{CFU} \cdot \mathrm{mL}^{-1}$, mainly $H$. influenzae and S. pneumoniae or $H$. influenzae and P. aeruginosa, in $60-80 \%$ of the cases [193, 206, 207]. There was an overall agreement between PSB and sputum cultures of $75 \%$. A good correlation was found between sputum cultures and bronchoscopic specimens in a recent study of patients with bronchiectasis [208].

In exacerbations of COPD, there is a lack of studies comparing sputum cultures with reference tests such as bronchoscopic procedures. SOLER et al. [196] compared PSB specimens with endotracheal aspirates and found a good correlation.

Four studies showed that during exacerbations the microbial bronchial flora increases significantly in nearly $50 \%$ of the patients [193, 196, 197, 209]. Authors classify the cultured organisms as potentially pathogenic microorganisms (PPM) and non-PPM [207]. On this basis it is accepted that bacterial pathogens cause $\sim 40-50 \%$ of the acute exacerbations of COPD.

Longitudinal studies have illustrated that as far as H. influenzae is concerned COPD exacerbations partly result from relapses by endogenous organisms, genomically identical to those of a previous exacerbation episode, and partly from exogenous reinfections with genomically different strains [194, 210].

Quality assessment of sputum was identical with that applied in the diagnosis of pneumonia. In a longitudinal study, ELLER et al. [199] found that in mild cases of COPD the sputum bacterial flora was mainly composed of: S. pneumoniae, Gram positive organisms $(46 \%)$, to a lesser degree $H$. influenzae and $M$. catarrhalis $(23 \%)$; and to a comparable degree
Enterobacteriaceae and P. aeruginosa (30\%) [199]. In severe cases with FEV $1<50 \%$ of the normal, the Gram negative organisms with $P$. aeruginosa became preponderant (63\%).

Using an arbitrary cut-off value of $10^{6} \mathrm{CFU} \cdot \mathrm{mL}^{-1}$ (and $10^{5}$ CFU $\cdot \mathrm{mL}^{-1}$ for S. pneumoniae), MirAvitLles et al. [198] also found a correlation between the degree of disease and the composition of the sputum flora. In the presence of FEV $1<50 \%$ cultures were significantly more positive for $H$. influenzae and $P$. aeruginosa. In patients with $\mathrm{FEV} 1>50 \%$ non-PPMs were isolated in most cases.

STOCKLEY et al. [15] classified sputa as either mucoid or purulent, on the basis of the myeloperoxidase content. This could also be evaluated by the colour of the sputum. The median bacterial load of mucoid sputum was $7.5 \times 10^{6}$ and that of purulent sputum was $10^{7}-10^{8} \mathrm{CFU} \cdot \mathrm{mL}^{-1}$. The presence of green purulent sputum was $94.4 \%$ sensitive and $77.0 \%$ specific for the yield of a high bacterial load.

HiLl et al. [211] studied several markers of inflammation together with bacterial loads, as well as the colour of the sputum. The myeloperoxidase level was low at bacterial loads $\leqslant 10^{7} \mathrm{CFU} \cdot \mathrm{mL}^{-1}$ ( $39 \%$ of the samples). Myeloperoxidase levels were high in the presence of bacteria $\pm 10^{7} \mathrm{CFU} \cdot \mathrm{mL}^{-1}(61 \%$ of the samples). In $92 \%$ of these specimens one bacterial species was predominant.

\section{Recommendation}

In patients with severe exacerbations of COPD (Group C patients), those who may have difficult-to-treat microorganisms ( $P$. aeruginosa) or potential resistances to antibiotics (prior antibiotic or oral steroid treatment, prolonged course of the disease, more than four exacerbations per year and FEV1 $<30 \%$ ), sputum cultures or endotracheal aspirates (in mechanically ventilated patients) are recommended (A3).

\section{Which initial antimicrobial treatments are recommended for} patients admitted to hospital with COPD exacerbation?

The more frequently isolated microorganisms in Group A are $H$. influenzae followed by S. pneumoniae and M. catarrhalis [212]. Amoxicillin, ampicillin or tetracycline are antibiotics that can be used, but in countries with high levels of antibiotic resistances of $S$. pneumoniae this may be a concern. In addition, $20-30 \%$ of strains of $H$. influenzae are $\beta$-lactamase producers and are resistant to penicillins. A retrospective study on COPD exacerbations found that amoxicillin was associated with a higher rate of relapse [205].

Amoxicillin-clavulanate is active against S. pneumoniae and $H$. influenzae. However, this antibiotic has to be used in high dosages (875 and $125 \mathrm{mg}$ amocicillin and clavulante, respectively) in order to obtain high concentrations in serum and in bronchial secretions above the MIC of the majority of the strains resistant to penicillin. The new formulation of 2,000 and 125 b.i.d (amocxicillin and clavulante, respectively) may be useful for this purpose. Resistances of $S$. pneumoniae to macrolides can be as high as $30-50 \%$ in some European countries (e.g. France, Spain); therefore, they are not generally recommended. In addition, most of the strains of $H$. influenzae are resistant to clarithromycin. However, most of the trials in this group of patients $[204,213]$ have shown a good effectiveness of macrolides compared with other antibiotics, 
which could be related to their anti-inflammatory effect. Telithromycin is a ketolide derived from macrolides that: 1) presents a low rate of resistance,; 2) is effective against $S$. pneumoniae resistant to penicillins and macrolides; 3 ) shows excellent antimicrobial activity against atypicals; and 4) is more effective than azithromycin against $H$. influenzae. It presents low side-effects, good penetration and excellent pharmacokinetic properties. It is also a potential antibiotic to be considered. However, the clinical experience is still limited [214]; therefore, it is not recommended (B2).

In Group B, in addition to amoxicillin-clavulanate, the new quinolones have to be considered. Levofloxacin and moxifloxacin are active against most of the strains of S. pneumoniae and $H$. influenzae and achieve high concentrations in bronchial secretions several times higher than the required MIC to treat these microorganisms [215-219]. In addition, they are active against Gram negative bacilli other than $P$. aeruginosa. These characteristics make these antibiotics very convenient to treat the exacerbations in this group of COPD patients. The oral route is preferred, but in some situations the parenteral route has to be used. In this case, the same antibiotics recommended above can be given parenterally. The administration of a single dosage every $24 \mathrm{~h}$ in the case of moxifloxacin is a potential advantage.

Nonantipseudomonal third-generation cephalosporins, such as ceftriaxone and cefotaxime, are also antibiotics with good activity against the majority of microorganisms. Within this group the antibiotics cause exacerbations and can be administered as monotherapy [220]. The advantage of ceftriaxone over cefotaxime is that it can be given intramuscularly and can be useful in some nonhospitalised cases.

In Group C the best orally administered anti-pseudomonal antibiotic is ciprofloxacin. This antibiotic is active against H. influenzae, M. catarrhalis and other Gram negative bacilli. A concern with ciprofloxacin is its poor activity against S. pneumoniae, but this microorganism is not frequent in patients from Group B. Another concern is the increasing rate of resistances to $P$. aeruginosa observed in some European countries. High dosages of ciprofloxacin are preferred $(750 \mathrm{mg}$ per $12 \mathrm{~h}$ ) to achieve higher serum and bronchial concentrations $[221,222]$. The activity of levofloxacin against $P$. aeruginosa has recently been approved by the Food and Drug Association (750 $\mathrm{mg}$ per $24 \mathrm{~h}$ ), although the clinical experience is limited and it is not recommended.

There is no clear information about when to use oral or i.v. route of administration in hospitalised patients. The oral route is preferred if the patient is able to eat. If this is not the case, the i.v. route has to be used and switched to oral when there is clinical stabilisation 3-5 days after admission. In the most severely ill patients (ICU admitted), i.v. administration of antibiotics is imperative. In this case and when Pseudomonas is suspected, combinations of antibiotics are advisable as mentioned in table 20. However, there is no evidence regarding the benefit of administration of two antibiotics to treat bronchial infections caused by $P$. aeruginosa. The duration of antibiotic treatment in COPD patients should be maintained at an average of 7-10 days. Courses of 5 days with levofloxacin or moxifloxacin have been as effective as 10 days of treatment with $\beta$-lactams in some trials [217, 223, 224].

\section{Recommendations}

In patients without risk factors for $P$. aeruginosa several options for antibiotic treatment are available. The selection of one or other antibiotic will depend on: 1) the severity of the exacerbation; 2) local pattern of resistances; 3) tolerability; 4) cost; and 5) potential compliance. Amoxicillin or tetracycline are recommended for mild exacerbations (usually managed at home) and co-amoxiclav in patients admitted to hospital with moderate-severe exacerbations (A2). Depending on the rate of pulmonary disease (COPD) exacerbations?

\begin{tabular}{|c|c|c|c|c|}
\hline Group & Definition & Oral treatment & Alternative & Parenteral treatment \\
\hline $\mathbf{A}^{\#}$ & Mild COPD without comorbidity & $\begin{array}{l}\text { Usually no treatment. If indication } \\
\text { then: amoxicillin, tetracycline }\end{array}$ & $\begin{array}{c}\text { Co-amoxiclav } \\
\text { Macrolide } \\
\text { Levofloxacin }^{+} \\
\text {Moxifloxacin }^{+}\end{array}$ & \\
\hline B & $\begin{array}{l}\text { Moderate-severe COPD without risk } \\
\text { factors for } P \text {. aeruginosa }\end{array}$ & Co-amoxiclav & $\begin{array}{l}\text { Levofloxacin } \\
\text { Moxifloxacin }\end{array}$ & $\begin{array}{c}\text { Amoxicillin-clavulanate, second or third } \\
\text { Generation cephalosporin }{ }^{\S} \text {, levofloxacin, } \\
\text { moxifloxacin }\end{array}$ \\
\hline$c^{\prime}$ & $\begin{array}{l}\text { Moderate or severe COPD with risk } \\
\text { factors for } P \text {. aeruginosa }\end{array}$ & Ciprofloxacin & & $\begin{array}{c}\text { Ciprofloxacin or } \beta \text {-lactam }{ }^{f} \text { with } \\
\text { P. aeruginosa activity } \pm \\
\text { aminoglycosides }{ }^{\# \#}\end{array}$ \\
\hline
\end{tabular}

P. aeruginosa: Pseudomonas aeruginosa. * : usually patients from Group A do not require hospitalisation. In countries with high incidence of Streptococcus pneumoniae resistant to penicillin, high dosages of amoxicillin or amoxicillin-clavulanate are recommended; $\uparrow$ : a benefit for empirical anti-pseudomonal antibiotic therapy in the presence for risk factors for $P$. aeruginosa is not proven; ${ }^{+}$: moxifloxacin and levofloxacin offer better coverage against $S$. pneumoniae than ciprofloxacin; ${ }^{\S}$ : ceftriaxone and cefotaxime; ${ }^{f}$ : cefepime, piperacillin-tazobactam or a carbapenem; ${ }^{\# \#}$ : there is no data on the benefit of combination therapy for $P$. aeruginosa treatment in exacerbated COPD patients. 
resistance to penicillin in S. pneumoniae in each country, the dosage of amoxicillin should be varied. In countries with a high resistance rate, the recommended dose is $1 \mathrm{~g}$ every $8 \mathrm{~h}$.

In patients with risk factors for $P$. aeruginosa, ciprofloxacin is the antibiotic of choice when the oral route is available. When parenteral treatment is needed, ciprofloxacin or a $\beta$-lactam with anti-pseudomonal activity are the available options. The addition of aminoglycosides is optional (A2).

The use of the oral or i.v. route depends on the stability of the clinical condition and the severity of exacerbation. Switching (i.v. to oral) is recommended by day 3 of admission if the patient is clinically stable (A3).

\section{How should the nonresponding patient be assessed?}

Between $10-20 \%$ of patients do not respond to empiric antimicrobial treatment [221, 222]. Some failures may be related to the following microbiological issues. 1) The initial infection is caused by a microorganism not covered by the empirical regimen. P. aeruginosa, Staph aureus (including methicillin-resistant Staph aureus), acinetobacter and other nonfermenters are the most frequent causes of failure. Aspergillus spp. has been described in some cases with prolonged treatment with steroids. High-level antibiotic resistance in S. pneumoniae also has to be considered. 2) The patient may have a nosocomial respiratory infection. This is more frequent in patients requiring invasive mechanical ventilation [196].

\section{Recommendation}

After close re-evaluation of noninfectious causes of failure (i.e. inadequate medical treatment, embolisms, cardiac failure, other) a careful microbiological reassessment, as mentioned in the section on microbiological diagnosis, is recommended (C3).

The recommendation for treatment in cases of failure includes an antibiotic change with good coverage against $P$. aeruginosa, S. pneumoniae resistant to antibiotics and nonfermenters, and to subsequently adjust the new antibiotic treatment according to microbiological results (C3).

\section{Exacerbations of bronchiectasis}

How should exacerbations of bronchiectasis be managed?

Bronchiectasis is a structural derangement of the bronchial wall that is characterised by airway dilatation and bronchial wall thickening. As a result of this abnormality, chronic inflammation and airway colonisation are characteristic findings. The aetiology of bronchiectasis is unknown in $50 \%$ of cases [225]. Tuberculosis, pneumonia, Ig deficiencies, bronchopulmonary aspergillosis and cystic fibrosis (CF) are the main causes of bronchiectasis. CF is outside the scope of these recommendations.

Colonisation with potential pathogenic microorganisms is identified in $60-80 \%$ of patients under stable clinical conditions. The most frequent microorganisms isolated are $H$. influenzae and Pseudomonas spp. [206, 208]. These microorganisms are probably those that cause periodic exacerbations. Other Gram negative bacilli, such as S. pneumoniae and Staph aureus, may also colonise the lower airways of these patients. It has been demonstrated that patients with bronchiectasis colonised by Pseudomonas spp. exhibit more extensive lung lesions (evidenced by high-resolution computed tomography) [226], suffer from more severe impairment of lung function [227, 228], and have a more intense inflammatory response in the lung [229]. Rarely, airway colonisation is due to microorganisms that require specific treatment, such as Nocardia asteroides, Aspergillus spp., Alcaligenes xylosoxidans and Mycobacterium spp. [208]. A recent study found an association between Staph aureus in the airways and the presence of allergic bronchopulmonary aspergillosis [230]. Potentially nonpathogenic microorganisms, such as $H$. parainfluenzae, are also frequently isolated from the sputum. Although its pathogenic role has been questioned, a recent study reported species-specific systemic immune responses to $H$. parainfluenzae [231].

The evidence of a more rapid progression of the disease when the airways are colonised by $P$. aeruginosa, the chances of having particular pathogens that require specific treatment, such as Aspergillus spp. or N. asteroids, and the demonstration of a more intense local and systemic inflammatory reaction in those patients colonised by potentially pathogenic microorganisms support the need for periodic evaluation of bronchial colonisation patterns in patients with bronchiectasis. Sputum cultures are as effective as bronchoscopy-guided techniques in evaluating colonisation in patients with bronchiectasis [208].

Acute exacerbations (see Appendix 1, Definitions section) are clinically recognised by an increased sputum production that becomes purulent and thicker. This increased purulence may be accompanied by other symptoms or signs, such as dyspnoea or increase in dyspnoea, cough and fever.

There is little information about microbiology in exacerbations of non-CF bronchiectasis. It is assumed that most of the exacerbations are caused by the colonising bacterial flora and therapy should, therefore, be directed towards these. Many patients with bronchiectasis take antibiotics regularly and this is a risk factor for bacterial antibiotic resistance. There are two additional recommendations for antibiotic treatment in patients with exacerbations. 1) Bacteriological flora and patterns of resistances are diverse and in a percentage of cases recent prior colonisation is not known. As a result, sputum cultures are recommended in most cases. 2) It is better to use antimicrobials with good bronchial penetration and the ability to decrease bacterial burden as much as possible. In addition, patients with bronchiectasis produce thick secretions at the site of microbial colonisation, which represent a physical barrier to drug diffusion.

On the basis of these considerations, the appropriate first-line policy would be to use antibiotics that are active against $H$. influenzae empirically, once a sputum sample for microbiological analysis has been taken. For oral ambulatory treatment, recommended antibiotics are amoxicillinclavulanate and quinolones (ciprofloxacin, moxifloxacin, levofloxacin). Levofloxacin has been effective in the management of outpatient bronchiectasis [232].

In case of risk factors for $P$. aeruginosa, ciprofloxacin is the best oral anti-pseudomonal agent and combinations of antibiotics may be advisable, as mentioned in table 21. Again, although there is no evidence regarding the benefit of administration of two antibiotics to treat acute exacerbations of bronchiectasis caused by $P$. aeruginosa. Levofloxacin can also be used on the 


\begin{tabular}{|c|c|c|c|}
\hline \multirow[t]{2}{*}{ TABLE 21} & \multicolumn{3}{|c|}{$\begin{array}{l}\text { Which antibiotics are recommended for } \\
\text { exacerbations of bronchiectasis? }\end{array}$} \\
\hline & & Oral Treatment & Parenteral Treatment \\
\hline \multirow{5}{*}{\multicolumn{2}{|c|}{$\begin{array}{l}\text { No risk of Pseudomonas } \\
\text { spp. }\end{array}$}} & $\begin{array}{l}\text { Amoxicillin- } \\
\text { clavulanate }\end{array}$ & Amoxicillin-clavulanate \\
\hline & & Moxifloxacin & Ceftriaxone \\
\hline & & Levofloxacin & Cefotaxime \\
\hline & & & Moxifloxacin \\
\hline & & & Levofloxacin \\
\hline \multicolumn{2}{|c|}{$\begin{array}{l}\text { Risk of Pseudomonas } \\
\text { spp. }^{\#}\end{array}$} & Ciprofloxacin & $\begin{array}{l}\text { Ciprofloxacin/Levofloxacin+ } \\
\text { anti-pseudomonal } \beta \text {-lactam } \\
\quad \text { or aminoglycoside }\end{array}$ \\
\hline \multicolumn{4}{|c|}{$\begin{array}{l}\text { \#: use the same criteria mentioned for chronic obstructive pulmonary } \\
\text { disease exacerbations; ' } \because \text { : ceftazidime, cefepime, carbapenems, piperacillin- } \\
\text { tazobactam. }\end{array}$} \\
\hline
\end{tabular}

basis of pharmacokinetic/pharmacodynamic information. Ceftriaxone, although not active against $P$. aeruginosa, can be used if ambulatory parenteral treatment is needed. Parenteral treatment should usually be reserved for patients needing hospitalisation with acute respiratory failure.

Risk factors for Pseudomonas spp. have not been extensively studied, but based on the observations above it would be reasonable to use the same four features as in COPD (see What are the risk factors for $P$. aeruginosa?). However, this has not been prospectively studied in relation to outcome.

Once they are available, empirical antibiotics should always be adjusted in accordance with microbiological results. Although there is no data on this issue, the duration of treatment should be 7-10 days, except in the case of $P$. aeruginosa infection where a longer duration may be needed (10-14 days).

Since patients with bronchiectasis will need antibiotics frequently, rotation of antibiotics in order to decrease the development of resistances may be advisable.

\section{Recommendation}

Periodical surveillance of colonisation is advisable (B3). The majority of patients with exacerbations will benefit from antibiotic treatment (B3). Obtaining a sputum sample for culture before starting antibiotic treatment is recommended in most cases and particularly in those requiring hospitalisation (B3). For empirical antibiotic treatment patients have to be stratified according to the potential risk of Pseudomonas spp. infection (B3; see What are the risk factors for $P$. aeruginosa?). Recommended antibiotics are summarised in table 21. Empirical antibiotics have to be adjusted or modified according to sputum culture results (A3).

\section{PREVENTION \\ Prevention by methods other than vaccination}

Does oral immunisation with bacterial extracts prevent LRTI?

$H$. influenzae is an important pathogen involved in infectious exacerbation of chronic bronchitis [194]. A systematic review identified six placebo-controlled, randomised studies investigating the efficacy of $H$. influenzae oral vaccine preparation to reduce the frequency of acute exacerbations (AE) in 440 patients with chronic bronchitis [233]. Overall there was a significant reduction of $\mathrm{AE}$ after 3 and 6 months of treatment compared with controls ( 3 months: Poisson rate ratio (PRR) $0.666,95 \%$ confidence interval (CI) $0.500-0.887, p=0.001 ; 6$ months: PRR 0.831, 95\% CI 0.669-1.031, $\mathrm{p}=0.093)$. The effect had disappeared by 9 months. Moreover, a reduction of the severity of $\mathrm{AE}$ at 3 and 6 months has also been shown $(-58 \%$, Peto odds ratio $(\mathrm{POR})=0.42$, and $95 \%$ CI $0.16-1.13$; and $-65 \%$, $\mathrm{POR}=0.35$, and $95 \%$ CI $0.16-0.75$, respectively). Although the authors of this review concluded that receiving the oral $H$. influenzae vaccine in the autumn reduces the frequency and severity of $\mathrm{AE}$ over the winter month, they admit that larger clinical trials are necessary to assess the long-term effects of this type of treatment.

Another approach is the use of a mixture of bacterial extracts as an oral vaccine (OM-85 BV). A placebo-controlled study investigated 350 nursing home residents aged $\geqslant 65 \mathrm{yrs}$ and found a significant reduction of acute bronchitis in patients in whom a mixed bacterial extract had been used $(28 \%$ reduction in the number of LRTIs (nonsignificant (NS)), $40 \%$ reduction in the number of episodes of acute bronchitis $(\mathrm{p}<0.01)$, and $28 \%$ reduction in the number of antibiotic prescriptions (NS)) [234]. However, the same study also showed a 39\% higher incidence of pneumonia and bronchopneumonia in the vaccinated group compared with the nonvaccinated group, suggesting that this approach provides no protection against severe types of pulmonary infections. Another study ( $\mathrm{n}=190$ OM-85 BV versus $n=191$ placebo) found no differences regarding the frequency of $\mathrm{AE}$, but a significant reduction in the total number of days hospitalised (OM-85 BV 287 days, placebo 642 days $(p=0.037)$ [235].

There are no cost-effectiveness studies on both substances so far.

\section{Recommendation}

The use of $H$. influenzae oral vaccine (B1) or of bacterial extracts (OM-85 BV; B2) in patients with $\mathrm{CB}$ or COPD is not recommended.

\section{What is the role of prophylactic antibiotic therapy in $C B$ and COPD?}

In $\mathrm{CB}$ and COPD patients the use of prophylactic antibiotic therapy has been studied extensively. Studies between 19601970 showed that prophylactic antimicrobial therapy, over months or years, is not effective in preventing infectious exacerbation [236-238].

\section{Recommendation}

On the basis of studies published so far, the prophylactic use of antibiotics in patients with $\mathrm{CB}$ or COPD as a matter of prevention is not recommended (A1).

According to the opinion of experts, it might be justified to use long-term antibiotic therapy in selected patients with bronchiectasis who suffer from frequent bacterial exacerbations, but no data from controlled studies are available for an evidencebased recommendation (C4).

The use of nebulised antibiotics for the prevention of LRTI in patients with bronchiectasis has also not been studied 
systematically so far. Therefore, no evidence for its use could be found and so the use of this approach is not recommended (C4).

\section{Does antibiotic treatment of URTIs prevent LRTI?}

Bacterial URTIs might be precursors of LRTIs. Only one study carried out in children investigated whether early antibiotic therapy of bacterial URTI could reduce the frequency of pneumonia and demonstrated that this was not the case [239]. The treatment of the common cold with antibiotics or antivirals also does not prevent LRTI [240, 241]. No evidence is available regarding the efficacy of the treatment of chronic sinusitis for the prevention of LRTI.

\section{Recommendation}

Treatment of URTIs with antibiotics will not prevent LRTIs (A1).

\section{Does the treatment with inhaled steroids or long-acting $\beta_{2}$-agonists prevent LRTI?}

Inhaled steroids might reduce the frequency of AECOPD in patients with severe airflow obstruction. However, no study has shown the prevention of LRTI by inhaled steroids in particular. Moreover, the reduction of AE in COPD seems to be limited to patients with severe COPD. For an overview regarding this question see the following guidelines [242, 243].

Regarding the use of long-acting $\beta_{2}$-agonist no evidence is available showing a preventive efficacy against LRTI.

\section{Recommendation}

The regular use of inhaled steroids (B1) or of a long-acting $\beta_{2^{-}}$ agonist (C4) as a preventive approach for LRTI is not recommended. This does not mean that they might not prevent exacerbations of COPD, which is an issue beyond the scope of this document.

\section{Does regular physiotherapy prevent LRTI?}

Although it seems justified to accept physiotherapy as part of good clinical practice for patients with hypersecretion and chronic airway disease, including bronchiectasis, no data support the use of physiotherapeutic approaches as a matter of prevention against LRTI [244].

\section{Recommendation}

Physiotherapy is not recommended as a preventive approach against LRTI (C4).

\section{Do anti-viral substances prevent influenza virus infection?}

Two classes of anti-virals for the prevention of influenza virus infection are available. Amantadine and rimantadine are M2 inhibitors with efficacy against the influenza A virus only, whereas the new substances zanamivir and oseltamivir as neuraminidase inhibitors are effective against both influenza A and $\mathrm{B}$.

A recent systematic review investigated 18 studies (14 amantadine, four rimantaine) including more than 10,000 patients [33]. Amantadine showed a significant preventive effectiveness of $63 \%$ (95\% CI: $42-76 \%$ ) in confirmed contacts of influenza A cases. Although the data for the effectiveness of rimantadine were comparable, a significant effect could not be obtained due to the smaller number of patients investigated. However, all available data showed that the frequency of side-effects is significantly lower with rimantadine than amantadine.

The neuraminidase inhibitors zanamivir (available only for inhalation) and oseltamivir (available only as oral medication) are less extensively studied [245-248]. Nevertheless, due to a more sophisticated design of the newer studies, a systematic review of the available data (eight studies: five zanamivir, three oseltamivir; $n=1,180$ ), showed a significant preventive effectiveness for both drugs (74\%; 95\% CI: 0.50-0.87) [249].

In summary, there is consistent evidence that amantadine, rimantadine and neuraminidase inhibitors have a preventive effect against influenza virus infections. However, vaccination against influenza virus infection is still the most important preventive method. There are no cost-effectiveness studies so far.

\section{Recommendation}

Prevention of influenza by anti-viral substances is only recommended in special situations; for example, in outbreaks in closed communities (A1).

\section{Are oral mucolytics useful for the prevention of $L R T I$ ?}

Mucolytic substances, such as acetylcysteine, carbocysteine and ambroxol, are widely used in patients with chronic bronchitis. A recent systematic review investigated 23 studies aimed to reduce the number of AEs of chronic obstructive bronchitis by mucolytic substances [250]. The authors found a weighted mean difference (WMD) for the reduction of AEs of -0.066 per month $(95 \%$ CI $-0.077--0.054 ; \mathrm{p}<0.001)$ or -0.84 $\mathrm{AE} \cdot \mathrm{yr}^{-1}$. Using the annualised rate of exacerbations in the control patients of $2.7 \cdot \mathrm{yr}^{-1}$, this gave a $29 \%$ reduction. The number of days of disability also fell (WMD -0.56; 95\% CI $-0.77--0.35 ; \mathrm{p}<0.001)$. The number of patients who remained exacerbation free was greater in the mucolytic group (odds ratio (OR) 2.22; 95\% CI 1.93-2.54; $\mathrm{p}<0.001$ ). The authors concluded that in subjects with $\mathrm{CB}$ or COPD, treatment with mucolytics was associated with a small reduction in acute exacerbations and a somewhat greater reduction in total number of days of disability. However, the studies did not show in particular a preventive effect against LRTI. This is also true for a systematic review investigating $\mathrm{N}$-acetylcysteine [251].

\section{Recommendation}

The regular use of oral mucolytics in patients with $\mathrm{CB}$ and COPD for prevention of LRTI is not recommended (B1).

The same is largely true for patients with bronchiectasis, where a systematic review included three trials, but could not aggregate their data in a meta-analysis [252]. Compared with placebo, high doses of bromhexine with antibiotics eased difficulty in expectoration (WMD -0.53 ; 95\% CI $-0.81--0.25$ ) and reduced sputum production by day 16 (WMD $-21.5 \%$; $95 \%$ CI -38.9- -4.1). Compared with placebo, recombinant human DNase also showed no preventive effect against LRTI in bronchiectasis patients. Influenza-like symptoms were more common in the group receiving recombinant human DNase [252]. 


\section{Recommendation}

The regular use of oral mycolytics in patients with bronchiectasis as a matter of prevention against LRTI is not recommended (B1).

\section{Is there evidence that homeopathic substances prevent LRTI?} A number of systematic reviews have addressed the question about the usefulness of different homeopathic substances for the prevention and treatment of the common cold and its complications. Substances include echinacea, zinc (nasal sprays, lozengers) and oscillococcinum derivates [253-257]. No review found any significant preventive effect.

No data are available showing that homeopathic substances have a preventive efficacy against LRTI.

\section{Recommendation}

The use of homeopathic substances is not recommended as a preventive approach against LRTI (C4).

\section{Prevention by vaccination}

\section{Influenza vaccine}

\section{History and presentation of the vaccine}

Most current influenza vaccines are based on "inactivated" viruses. There are three types: whole virion vaccines; subunit virion vaccines; and split virion vaccines. Whole virion vaccines consist of whole inactivated or killed virus, subunit vaccines of the $\mathrm{H}$ and $\mathrm{N}$ surface antigens only, and split virion vaccines of a viral structure that has been broken up and which contains both internal and surface antigens. In addition, there are a variety of live attenuated vaccines, mostly in nonEuropean countries, that can be administered noninvasively by nasal application.

\section{What is the immune response to the vaccine/vaccines?}

In immunogenicity studies of influenza vaccines a haemagglutinin-inhibiting (HI) titre of $\geqslant 40$ is often used as a cut-off level, as that level is considered to be a good surrogate marker for protective efficacy against clinical influenza [258]. However, the predictive value of this surrogate marker may be questioned. In a randomised controlled trial in a nursing home, with an inactivated trivalent vaccine versus a diphtheria toxoid conjugate influenza vaccine, $50 \%$ of those who developed laboratory-verified influenza had an $\mathrm{HI}$ titre $>1: 40$ and $30 \%$ had a titre $>1: 640$ a month after immunisation [259]. Bearing that in mind, it has been well documented that inactivated influenza vaccines induce a "protective" HI titre against Influenza A and B in 70-100\% of healthy adults [258]. In the elderly, the response is not as good. Between $30-70 \%$ reach an HI titre of $>1: 40$ [260-263]. In these age groups, a double dose, or a booster dose, has been shown to increase the geometric mean IgG antibody concentration by $\sim 15 \%$ [264]. Live attenuated vaccines do not reach as high $\mathrm{HI}$ titres as the inctivated, but induce significantly higher local IgA titres in the nasal mucosa [258].

\section{Are the vaccines safe?}

Inactivated and live attenuated influenza vaccines are generally very safe, with systemic reactions in $\sim 6 \%$ of those vaccinated [258]. Systemic symptoms or disability are seen as often in persons receiving placebo as in those who have received inactivated influenza vaccine. Mild local reactions, such as a "sore arm", are less common after placebo than after active substance, $5-10 \%$ versus $15-20 \%$, respectively [265-267]. The inactivated vaccine is also safe to administer to patients with asthma, including those with severe asthma [268]. Inactivated split-virion vaccines present less frequent local reactions than whole virion vaccines [269]. In a large population-based study, using a split-virion vaccine, local reactions were seen in only $6 \%$ of 4,581 vaccinated elderly subjects and there were only 10 cases $(0.2 \%)$ of fever [270]. Also, after nasal administration of live attenuated vaccine local reactions such as "runny nose" occur more commonly in the active than in the placebo group, $\sim 40 \%$ versus $25 \%$, respectively [271].

\section{What is the efficacy/effectiveness of the vaccine/vaccines?}

In a Cochrane analysis, including 20 studies with 26,369 vaccinated subjects, the preventive efficacy of serologically verified influenza in adults aged 14-60 yrs was 48\% (95\% CI 24-64) for live attenuated aerosol vaccines and 68\% (95\% CI 49-79) for inactivated vaccines [272]. For clinical influenza the efficacy was smaller, 13 and 24\%, respectively, and although vaccines significantly reduced time off work, it was only by 0.4 days (95\% CI 0.1-0.8). A more recent meta-analysis, including data from 11 trials with 4,088 vaccinated subjects, indicated that the live attenuated and the inactivated vaccine were equally effective, $\sim 70-80 \%$, in prevention of culture-positive influenza in subjects aged 1-65 yrs [258].

In the elderly, only one randomised placebo-controlled trial has been performed [273]. This trial consisted of 1,838 persons aged $\geqslant 60 \mathrm{yrs}$, without any high-risk conditions. A split-virion vaccine conferred significant protection against serologically confirmed influenza $(50 \%)$, clinical influenza $(47 \%)$, and against the combination of serological and clinical influenza $(58 \%)$. Many cohort and case-control studies have investigated the efficacy of influenza vaccine in the prevention of hospitalisation for influenza-related end-points, such as influenza and pneumonia, but also for other outcomes, e.g. heart failure and chronic respiratory disease. According to a meta-analysis of 20 cohort studies, published between 1974 and 1992, the pooled estimate of the efficacy of the vaccine was: $56 \%$ for prevention of respiratory illness; $53 \%$ for prevention of pneumonia; $50 \%$ for prevention of hospitalisation overall; and $68 \%$ for prevention of death [274]. Recent large cohort studies of the elderly in the community have confirmed these findings, demonstrating efficacy rates between $20-80 \%$ in the prevention of hospitalisation for influenza and pneumonia, $40 \%$ in the prevention of congestive heart failure, and $40-70 \%$ in the prevention of death overall [275-280]. There are also data indicating that the absolute risk reduction is 2-5 times higher among high-risk persons than among healthy elderly persons [281]. A protective efficacy against influenza, pneumonia, and death of the same magnitude has also been demonstrated in the nursing home setting [282-284]. There is some evidence that influenza vaccination of healthcare personnel may reduce mortality of elderly people in long-term hospitals, although no difference in the proportion of elderly persons positive for influenza was seen $[285,286]$. 


\section{Is vaccination cost-effective?}

In healthy adults the results of cost-effectiveness studies have varied. One study demonstrated a net cost of USA $\$ 68.00$ per vaccinated subject in a year with a poor match between vaccine and infecting strains, as compared with a net cost of USA $\$ 11.00$ during the following year with a good vaccine match [287]. Other studies have found vaccination to be cost saving [288, 289]. In elderly persons, vaccination has also been cost-saving $[275,290-292]$. Vaccination is probably also cost-saving relative to providing anti-viral treatment with neuraminidase inhibitors [293].

What is the safety, immunogenicity and efficacy of revaccination? Annually repeated vaccinations do not lead to a decreasing immune response or protection [294]. Although much less studied, repeated vaccinations annually, or even more frequent, do not lead to more side-effects than after primary vaccination [294, 295].

\section{Recommendations for influenza vaccination \\ Should influenza vaccine be used to prevent LRTI?}

The influenza vaccine should be given yearly to persons at increased risk for complications due to influenza (A1). Vaccination is recommended for immunocompetent adults belonging to one, or more, of the following categories: 1) aged $\geqslant 65$ yrs; 2) institutionalisation; 3) chronic cardiac diseases; 4) chronic pulmonary diseases; 5) diabetes mellitus; 6) chronic renal diseases; 7) haemoglobinopathies; and 7) females who will be in the second or third trimester of pregnancy during the influenza season [296]. Repeated vaccinations are safe and do not lead to a decreased immune response (B1). In adults the inactivated, rather than the live attenuated, vaccine is recommended (A1). In health-care personnel yearly vaccination is recommended, especially in settings where elderly persons or other high-risk groups are treated (B2). In the absence of robust cost-effectiveness data for vaccination of all healthy adults, a general vaccination of this group cannot be recommended.

\section{Pneumococcal vaccination}

History and presentation of the vaccine

There are currently two pneumococcal vaccines available, a 23valent (containing 23 serotypes) capsular polysaccharide vaccine, representing $\sim 90 \%$ of all serotypes that cause invasive pneumococcal disease, and a heptavalent protein-polysaccharide conjugate vaccine. The polysaccharide vaccine has been available for $>15$ yrs. The conjugate vaccine has recently been licensed in some countries, and is specially designed for paediatric use.

What are the risk factors for pneumococcal disease?

There are very few modern studies on risk factors for pneumococcal disease in immunocompetent adults. In a casecontrol study in a presumed high-risk population (mostly elderly males), dementia, seizure disorders, current cigarette smoking, congestive heart failure, cerebrovascular disease, institutionalisation and COPD were independent risk factors for developing a culture-proven pneumococcal infection [297]. Risk increased with age and previous hospitalisation. In healthy immunocompetent adults, aged 18-64 yrs, cigarette smoking, both active and passive, were the major risk factors for invasive pneumococcal disease [298]. Several other factors that are considered as increasing the risk factors for pneumococcal disease have been described, such as aged $\geqslant 65 \mathrm{yrs}$, chronic liver disease, diabetes mellitus, functional or anatomic asplenia, and chronic cerebrospinal fluid leakage [299].

\section{What is the immune response to the vaccine/vaccines?}

The antibody $(\mathrm{IgG})$ rise after vaccination with pneumococcal polysaccharide vaccine in the elderly is generally good, and comparable with that of younger persons [300-305]. However, $\sim 20 \%$ of elderly subjects have a poor response, both quantitatively and qualitatively. From the peak level, $\sim 1$ month after vaccination, there is a gradual decline of antibody levels back to pre-vaccination levels. The duration of the antibody rise has varied, from 3-4 yrs to >5-10 yrs in different studies [306, 307]. There is no known "protective level" of IgG antibodies or any other "surrogate markers" of protection against pneumococcal disease. However, high levels and especially the capacity of responding with an antibody fold increase of four, or more, is associated with a preventive efficacy against pneumonia [308].

In the elderly, a single injection of protein-conjugated polysaccharide vaccine does not elicit a better antibody response than polysaccharide vaccine alone, and vaccination with protein-conjugated vaccine followed by polysaccharide vaccine alone, does not seem to induce a booster effect [309-312].

\section{Is the vaccine safe?}

Primary vaccination with the polysaccharide vaccine is safe and although local reactions are somewhat more common than after influenza vaccination, serious reactions are extremely rare $[270,313]$. Although a slight soreness and/or redness or swelling may occur in up to $50 \%$ of the cases, this is mostly very mild and short-lived [313]. Severe soreness, swelling $(>10 \mathrm{~cm})$ or redness occurs in only $2-3 \%$ and resolves within a few days.

What is the efficacy/effectiveness of the vaccine/vaccines?

The efficacy of the pneumococcal polysaccharide vaccine has been evaluated in five meta-analyses, or systematic reviews [314-318]. The quality, as well as the results, of these metaanalyses/systematic reviews differs, due to when they were performed and differences in methodology. Thus, evidence levels vary from $\mathrm{A} 1+$ to $\mathrm{C} 1-$. The five studies have a low power to determine the effectiveness of the vaccine in prevention of invasive pneumococcal disease (IPD) in the elderly. This is because none of the randomised prospective studies were designed to study this end-point, resulting in a total of $<20$ elderly with IPD being included in the systematic reviews.

The meta-analyses/systematic reviews cited above have clearly shown that the polysaccharide vaccine prevents against bacteraemic pneumococcal pneumonia (70-80\% effective) in young healthy adults, and also against presumptive pneumococcal pneumonia and death in pneumonia overall. This finding is mostly based on studies performed in South African gold miners during the 1970s [319, 320]

The meta-analyses/systematic reviews have not shown that the elderly would have any benefit from pneumococcal vaccination. However, in one meta-analysis there was a $42 \%$ 
reduction of the risk for definitive pneumococcal pneumonia in vaccinated persons aged $\geqslant 55$ yrs (OR $0.58 ; 95 \%$ CI $0.18-1.0$ ) [317]. This finding was based on only 11 IPD cases (three in vaccinated versus eight in nonvaccinated elderly subjects). Of those, six were from the only large study in which blood cultures were obtained in a systematic way [321]. If this metaanalysis had also included the large quasi-randomised study by HONKANEN et al. [322], with two cases in the vaccinated group versus five in the control group, a significant protective efficacy of $\sim 60 \%$ would probably have been demonstrated. An efficacy of the vaccine against IPD in the immune-competent elderly at that same level $(60-80 \%)$ has been indicated in several case-control and cohort studies [323-327].

There is no evidence from randomised studies and systematic reviews that the polysaccharide vaccine prevents against all pneumonia. However, a recent retrospective cohort study in elderly persons with COPD indicated that pneumococcal vaccination was associated with a $43 \%$ reduction of hospitalisation for pneumonia, compared with nonvaccinated persons [328].

\section{Are the vaccines cost-effective?}

A recent study of the cost-effectiveness of preventing pneumococcal pneumonia in young military personnel in the USA, found the vaccine to be cost-saving if the incidence of pneumococcal pneumonia was 2.2 per $1,000 \cdot \mathrm{yr}^{-1}$ and a vaccine efficacy of $70 \%$ was assumed [329]. This efficacy level was based on the studies performed on South African gold miners. However, a sensitivity analysis indicated that the vaccine should still be cost-saving even if the efficacy was lowered to $50 \%$.

In the elderly, recent cost-effectiveness analyses of the polysaccharide vaccine have focused on prevention of invasive pneumococcal disease, since there is no evidence for the vaccine being effective in the prevention of pneumonia. In the USA, the vaccine was found to be cost-saving in the prevention of IPD in persons aged $\geqslant 65$ yrs [330]. This study was based on the efficacy data from the case-control study by SHAPIRO et al. [325]. Cost-effective analyses from the USA and from Europe are difficult to compare because of significant differences regarding organisation and costs for healthcare. In two European studies, the vaccine has not been cost-saving, but moderately cost-effective. The first, which also used the efficacy data from SHAPIRO et al. [325], was based on epidemiological data from five European countries: Belgium, France, Scotland (UK), Spain and Sweden. It demonstrated that the cost-effectiveness ratios varied from 11,000-33,000 Euros per quality-adjusted life year in preventing hospital admission for IPD [331]. The second, based on Dutch epidemiological data and efficacy data from several studies, found approximately the same cost-effectiveness with a net cost of 10,100 Euros per life year gained [332].

What is the safety, immunogenicity and efficacy of revaccination? Revaccination of immune-competent adults with the pneumococcal polysaccharide vaccine has not been extensively studied. Nothing is known about the efficacy of revaccination, but there are a few studies on the immune response and safety. Local reactions, mostly mild, are more common after revaccination rather than after primary vaccination, but fever or severe adverse events are rare. The frequency of major local reactions has varied between $10-15 \%$, and the risk seems to be higher in younger age groups and in persons with high prevaccination antibody levels [313, 333].

Most studies on the response to revaccination have been performed with older, less specific radio-immunoassay techniques. These studies have indicated that the revaccination response may be not as good as after primary vaccination. However, in a recent large study the antibody response after revaccination was lower than after primary vaccination for only one of three serotypes measured [313].

\section{Recommendations for pneumococcal vaccination}

Should pneumococcal vaccine be used to prevent LRTI?

The evidence for vaccination with the 23-valent polysaccharide pneumococcal vaccine is not as strong as that for influenza vaccination, but it is recommended that the vaccine be given to all adults at risk for pneumococcal disease (B4).

Risk factors for pneumococcal disease are: 1) aged $\geqslant 65 \mathrm{yrs}$; 2) institutionalisation; 3) dementia; 4) seizure disorders; 5) congestive heart failure; 6) cerebrovascular disease; 7) COPD; 8) history of a previous pneumonia; 9) chronic liver disease; 10) diabetes mellitus; 11) functional or anatomic asplenia; and 12) chronic cerebrospinal fluid leakage (B3). Although smoking seems to be a significant risk factor in otherwise healthy younger adults, measures aimed at reducing smoking and exposure to environmental tobacco smoke are preferable in this group.

Revaccination once can be considered in the elderly, 5-10 yrs after primary vaccination (B3).

\section{Vaccine uptake}

What is the best way to implement influenza and pneumococcal vaccination policies?

Both official recommendations and coverage of influenza and pneumococcal vaccination vary significantly between countries. Probably one of the most important factors for a good uptake is that there are family GPs who recommend vaccination $[334,335]$. However, fear of adverse effects of vaccination effectively lowers the vaccination rates.

For both influenza and pneumococcal vaccines, there is reasonably good evidence that interventions can significantly improve vaccine coverage among the elderly and other highrisk groups [336, 337]. Such interventions include measures to increase demand for, and enhance access to, immunisation, as well as provider-based interventions. It is not clear which type of intervention is the most cost-effective. In a meta-analysis of 16 studies into the effectiveness of interventions to increase influenza immunisation rates, the vaccination rate was approximately twice as high in the intervention group than among controls, irrespective of the method used (patientfocused, provider-focused or mixed) [338]. However, in elderly and high-risk outpatients, home visits, personal reminders and/or vaccinations given for free may be the most effective ways to improve vaccine uptake [339-343]. Interventions to increase vaccine uptake, e.g. standing orders, chart stickers and the provision of reminders, have also been shown to be effective in hospitalised patients, both in acute care settings [344] and in long-term facilities [345]. 
Improvement in influenza vaccine coverage in healthcare personnel seems to be difficult. During a vaccine campaign, directed towards primary healthcare teams and nursing home staff, everybody was offered free vaccination; in addition one group was randomised to receive educational visits by a public health nurse to raise awareness of the campaign [346]. However, no difference was seen between the two groups and the uptake was generally very low, 6-10\% in nursing home staff and $\sim 20 \%$ in the primary health-care teams.

\section{Recommendations for implementation}

Active interventions to enhance vaccination with either or both vaccines is effective and necessary to achieve an adequate vaccination coverage of the targeted population (B1).

\section{REFERENCES}

1 ERS Task Force Report. Guidelines for management of adult community-acquired lower respiratory tract infections. European Respiratory Society. Eur Respir J 1998; 11: 986-991.

2 Davie AP, Francis CM, Caruana L, Sutherland GR, McMurray JJ. Assessing diagnosis in heart failure: which features are any use? Q J Med 1997; 90: 335-339.

3 Cost B, Grobbee DE, Mostert A, Hoes AW. Detection of heart failure in elderly in general practice. Erasmus University, Amsterdam, Netherlands, 2000.

4 Wells PS, Anderson DR, Rodger M, et al. Excluding pulmonary embolism at the bedside without diagnostic imaging: management of patients with suspected pulmonary embolism presenting to the emergency department by using a simple clinical model and d-dimer. Ann Intern Med 2001; 135: 98-107.

5 Thiadens HA, de Bock GH, Dekker FW, et al. Identifying asthma and chronic obstructive pulmonary disease in patients with persistent cough presenting to general practitioners: descriptive study. BMJ 1998; 316: 1286-1290.

6 Hallett JS, Jacobs RL. Recurrent acute bronchitis: the association with undiagnosed bronchial asthma. Ann Allergy 1985; 55: 568-570.

7 Williamson HA Jr, Schultz P. An association between acute bronchitis and asthma. J Fam Pract 1987; 24: 35-38.

8 Smyrnios NA, Irwin RS, Curley FJ, French CL. From a prospective study of chronic cough: diagnostic and therapeutic aspects in older adults. Arch Intern Med 1998; 158: 1222-1228.

9 Palombini BC, Villanova CA, Araujo E, et al. A pathogenic triad in chronic cough: asthma, postnasal drip syndrome, and gastroesophageal reflux disease. Chest 1999; 116: 279-284.

10 McGarvey LP, Heaney LG, Lawson JT, et al. Evaluation and outcome of patients with chronic non-productive cough using a comprehensive diagnostic protocol. Thorax 1998; 53: 738-743.

11 Woodhead MA, Macfarlane JT, McCracken JS, Rose DH, Finch RG. Prospective study of the aetiology and outcome of pneumonia in the community. Lancet 1987; 1: 671-674.

12 Metlay JP, Kapoor WN, Fine MJ. Does this patient have community-acquired pneumonia? Diagnosing pneumonia by history and physical examination. JAMA 1997; 278: 1440-1445.

13 Melbye H, Straume B, Aasebo U, Brox J. The diagnosis of adult pneumonia in general practice. The diagnostic value of history, physical examination and some blood tests. Scand J Prim Health Care 1988; 6: 111-117.

14 Melbye H, Straume B, Brox J. Laboratory tests for pneumonia in general practice: the diagnostic values depend on the duration of illness. Scand J Prim Health Care 1992; 10: 234-240.

15 Stockley RA, O'Brien C, Pye A, Hill SL. Relationship of sputum color to nature and outpatient management of acute exacerbations of COPD. Chest 2000; 117: 1638-1645.

16 Dev D, Wallace E, Sankaran R, et al. Value of C-reactive protein measurements in exacerbations of chronic obstructive pulmonary disease. Respir Med 1998; 92: 664-667.

17 Hedlund J, Hansson LO. Procalcitonin and C-reactive protein levels in community-acquired pneumonia: correlation with etiology and prognosis. Infection 2000; 28: 68-73.

18 Jonsson JS, Sigurdsson JA, Kristinsson KG, Guthnadottir M, Magnusson S. Acute bronchitis in adults. How close do we come to its aetiology in general practice? Scand J Prim Health Care 1997; 15: 156-160.

19 Reed WW, Byrd GS, Gates RH Jr, Howard RS, Weaver MJ. Sputum gram's stain in community-acquired pneumococcal pneumonia. A meta-analysis. West J Med 1996; 165: 197-204.

20 Pavesi L, Subburaj S, Porter-Shaw K. Application and validation of a computerized cough acquisition system for objective monitoring of acute cough: a meta-analysis. Chest 2001; 120: 1121-1128.

21 Schroeder K, Fahey T. Over-the-counter medications for acute cough in children and adults in ambulatory settings. Cochrane Database Syst Rev 2001; 3: CD001831.

22 Matthys H, Erhardt J, Ruhle KH. [Objectivation of the effect of antitussive agents using tussometry in patients with chronic cough]. Schweiz Med Wochenschr 1985; 115: 307-311.

23 Smucny JJ, Flynn CA, Becker LA, Glazier RH. Are beta2agonists effective treatment for acute bronchitis or acute cough in patients without underlying pulmonary disease? A systematic review. J Fam Pract 2001; 50: 945-951.

24 Smucny J, Fahey T, Becker L, Glazier R, McIsaac W. Antibiotics for acute bronchitis. Cochrane Database Syst Rev 2000; 4: CD000245.

25 Evans AT, Husain S, Durairaj L, Sadowski LS, CharlesDamte M, Wang Y. Azithromycin for acute bronchitis: a randomised, double-blind, controlled trial. Lancet 2002; 359: 1648-1654.

26 Verheij TJ, Hermans J, Mulder JD. Effects of doxycycline in patients with acute cough and purulent sputum: a double blind placebo controlled trial. Br J Gen Pract 1994 44: 400-404.

27 Saint S, Bent S, Vittinghoff E, Grady D. Antibiotics in chronic obstructive pulmonary disease exacerbations. A meta-analysis. JAMA 1995; 273: 957-960.

28 Allegra L, Blasi F, de Bernardi B, Cosentini R, Tarsia P. Antibiotic treatment and baseline severity of disease in acute exacerbations of chronic bronchitis: a re-evaluation 
of previously published data of a placebo-controlled randomized study. Pulm Pharmacol Ther 2001; 14: 149-155.

29 Bach PB, Brown C, Gelfand SE, McCrory DC. Management of acute exacerbations of chronic obstructive pulmonary disease: a summary and appraisal of published evidence. Ann Intern Med 2001; 134: 600-620.

30 Jorgensen AF, Coolidge J, Pedersen PA, Petersen KP, Waldorff S, Widding E. Amoxicillin in treatment of acute uncomplicated exacerbations of chronic bronchitis. A double-blind, placebo-controlled multicentre study in general practice. Scand J Prim Health Care 1992; 10: 7-11.

31 Sachs AP, Koeter GH, Groenier KH, van der WD, Schiphuis J, Meyboom-de Jong B. Changes in symptoms, peak expiratory flow, and sputum flora during treatment with antibiotics of exacerbations in patients with chronic obstructive pulmonary disease in general practice. Thorax 1995; 50: 758-763.

32 Anthonisen NR, Manfreda J, Warren CP, Hershfield ES, Harding GK, Nelson NA. Antibiotic therapy in exacerbations of chronic obstructive pulmonary disease. Ann Intern Med 1987; 106: 196-204.

33 Jefferson TO, Demicheli V, Deeks JJ, Rivetti D. Amantadine and rimantadine for preventing and treating influenza A in adults. Cochrane Database Syst Rev 2002; 3: CD001169.

34 Guay DR. Amantadine and rimantadine prophylaxis of influenza A in nursing homes. A tolerability perspective. Drugs Aging 1994; 5: 8-19.

35 McNicholl IR, McNicholl JJ. Neuraminidase inhibitors: zanamivir and oseltamivir. Ann Pharmacother 2001; 35: 57-70.

36 Monto AS, Webster A, Keene O. Randomized, placebocontrolled studies of inhaled zanamivir in the treatment of influenza A and B: pooled efficacy analysis. J Antimicrob Chemother 1999; 44: Suppl. B, 23-29.

37 Fine MJ, Auble TE, Yealy DM, et al. A prediction rule to identify low-risk patients with community-acquired pneumonia. N Engl J Med 1997; 336: 243-250.

38 Neill AM, Martin IR, Weir R, et al. Community-acquired pneumonia: aetiology and usefulness of severity criteria on admission. Thorax 1996; 51: 1010-1016.

39 Lim WS, Lewis S, Macfarlane JT. Severity prediction rules in community-acquired pneumonia: a validation study. Thorax 2000; 55: 219-223.

40 Ewig S, Kleinfeld T, Bauer T, Seifert K, Schafer H, Goke N. Comparative validation of prognostic rules for community-acquired pneumonia in an elderly population. Eur Respir J 1999; 14: 370-375.

41 Roson B, Carratala J, Dorca J, Casanova A, Manresa F, Gudiol F. Etiology, reasons for hospitalization, risk classes, and outcomes of community-acquired pneumonia in patients hospitalized on the basis of conventional admission criteria. Clin Infect Dis 2001; 33: 158-165.

42 Marrie TJ, Lau CY, Wheeler SL, Wong CJ, Vandervoort MK, Feagan BG. A controlled trial of a critical pathway for treatment of community-acquired pneumonia. CAPITAL Study Investigators. CommunityAcquired Pneumonia Intervention Trial Assessing Levofloxacin. JAMA 2000; 283: 749-755.
43 Lim WS, Macfarlane JT, Boswell TC, et al. Study of community-acquired pneumonia aetiology (SCAPA) in adults admitted to hospital: implications for management guidelines. Thorax 2001; 56: 296-301.

44 Community-acquired pneumonia in adults in British hospitals in 1982-1983: a survey of aetiology, mortality, prognostic factors and outcome. The British Thoracic Society and the Public Health Laboratory Service. Q J Med 1987; 62: 195-220.

45 Farr BM, Sloman AJ, Fisch MJ. Predicting death in patients hospitalized for community-acquired pneumonia. Ann Intern Med 1991; 115: 428-436.

46 Karalus NC, Cursons RT, Leng RA, et al. Communityacquired pneumonia: aetiology and prognostic index evaluation. Thorax 1991; 46: 413-418.

47 Marras TK, Gutierrez C, Chan CK. Applying a prediction rule to identify low-risk patients with communityacquired pneumonia. Chest 2000; 118: 1339-1343.

48 Ewig S, Ruiz M, Mensa J, et al. Severe communityacquired pneumonia. Assessment of severity criteria. Am J Respir Crit Care Med 1998; 158: 1102-1108.

49 Minogue MF, Coley CM, Fine MJ, Marrie TJ, Kapoor WN, Singer DE. Patients hospitalized after initial outpatient treatment for community-acquired pneumonia. Ann Emerg Med 1998; 31: 376-380.

50 Porath A, Schlaeffer F, Lieberman D. Appropriateness of hospitalization of patients with community-acquired pneumonia. Ann Emerg Med 1996; 27: 176-183.

51 Niederman MS, Mandell LA, Anzueto A, et al. Guidelines for the management of adults with community-acquired pneumonia. Diagnosis, assessment of severity, antimicrobial therapy, and prevention. Am J Respir Crit Care Med 2001; 163: 1730-1754.

52 Angus DC, Marrie TJ, Obrosky DS, et al. Severe community-acquired pneumonia: use of intensive care services and evaluation of American and British Thoracic Society diagnostic criteria. Am J Respir Crit Care Med 2002; 166: 717-723.

53 Atlas SJ, Benzer TI, Borowsky LH, et al. Safely increasing the proportion of patients with community-acquired pneumonia treated as outpatients: an interventional trial. Arch Intern Med 1998; 158: 1350-1356.

54 Dean NC, Silver MP, Bateman KA, James B, Hadlock CJ, Hale D. Decreased mortality after implementation of a treatment guideline for community-acquired pneumonia. Am J Med 2001; 110: 451-457.

55 Levin KP, Hanusa BH, Rotondi A, et al. Arterial blood gas and pulse oximetry in initial management of patients with community-acquired pneumonia. J Gen Intern Med 2001; 16: 590-598.

56 Smith RP, Lipworth BJ, Cree IA, Spiers EM, Winter JH. Creactive protein. A clinical marker in communityacquired pneumonia. Chest 1995; 108: 1288-1291.

57 Hedlund J, Hansson LO. Procalcitonin and C-reactive protein levels in community-acquired pneumonia: correlation with etiology and prognosis. Infection 2000; 28: 68-73.

58 Theerthakarai R, El Halees W, Ismail M, Solis RA, Khan MA. Nonvalue of the initial microbiological studies in the management of nonsevere community-acquired pneumonia. Chest 2001; 119: 181-184. 
59 Sanyal S, Smith PR, Saha AC, Gupta S, Berkowitz L, Homel P. Initial microbiologic studies did not affect outcome in adults hospitalized with communityacquired pneumonia. Am J Respir Crit Care Med 1999; 160: 346-348.

60 Lieberman D, Schlaeffer F, Boldur I, et al. Multiple pathogens in adult patients admitted with communityacquired pneumonia: a one year prospective study of 346 consecutive patients. Thorax 1996; 51: 179-184.

61 Ruiz M, Ewig S, Marcos MA, et al. Etiology of community-acquired pneumonia: impact of age, comorbidity, and severity. Am J Respir Crit Care Med 1999; 160: 397-405.

62 Waterer GW, Somes GW, Wunderink RG. Monotherapy may be suboptimal for severe bacteremic pneumococcal pneumonia. Arch Intern Med 2001; 161: 1837-1842.

63 Marrie TJ, Haldane EV, Faulkner RS, Durant H, Kwan C. Community-acquired pneumonia requiring hospitalization. Is it different in the elderly? J Am Geriatr Soc 1985; 33: 671-680.

64 Marston BJ, Plouffe JF, File TM Jr, et al. Incidence of community-acquired pneumonia requiring hospitalization. Results of a population-based active surveillance Study in Ohio. The Community-Based Pneumonia Incidence Study Group. Arch Intern Med 1997; 157: 1709-1718.

65 Macfarlane JT, Colville A, Guion A, Macfarlane RM, Rose DH. Prospective study of aetiology and outcome of adult lower-respiratory-tract infections in the community. Lancet 1993; 341: 511-514.

66 Bishara J, Leibovici L, Ashkenazi S, Samra Z, Pitlik S. Seven-year study of bacteraemic pneumonia in a single institution. Eur J Clin Microbiol Infect Dis 2000; 19: 926-931.

67 Kalin M, Lindberg AA. Diagnosis of pneumococcal pneumonia: a comparison between microscopic examination of expectorate, antigen detection and cultural procedures. Scand J Infect Dis 1983; 15: 247-255.

68 Marrie TJ, Durant H, Yates L. Community-acquired pneumonia requiring hospitalization: 5-year prospective study. Rev Infect Dis 1989; 11: 586-599.

69 Gleckman R, DeVita J, Hibert D, Pelletier C, Martin R. Sputum gram stain assessment in community-acquired bacteremic pneumonia. J Clin Microbiol 1988; 26: 846-849.

70 Skerrett SJ. Diagnostic testing for community-acquired pneumonia. Clin Chest Med 1999; 20: 531-548.

71 Ruiz-Gonzalez A, Nogues A, Falguera M, Porcel JM, Huelin E, Rubio-Caballero M. Rapid detection of pneumococcal antigen in lung aspirates: comparison with culture and PCR technique. Respir Med 1997; 91: 201-206.

72 Ruiz-Gonzalez A, Falguera M, Nogues A, RubioCaballero M. Is Streptococcus pneumoniae the leading cause of pneumonia of unknown etiology? A microbiologic study of lung aspirates in consecutive patients with community-acquired pneumonia. Am J Med 1999; 106: 385-390.

73 Garcia A, Roson B, Perez JL, et al. Usefulness of PCR and antigen latex agglutination test with samples obtained by transthoracic needle aspiration for diagnosis of pneumococcal pneumonia. J Clin Microbiol 1999; 37: 709-714.
74 Scott JA, Hall AJ. The value and complications of percutaneous transthoracic lung aspiration for the etiologic diagnosis of community-acquired pneumonia. Chest 1999; 116: 1716-1732.

75 Ishida $\mathrm{T}$, Hashimoto $\mathrm{T}$, Arita $\mathrm{M}$, et al. Efficacy of transthoracic needle aspiration in community-acquired pneumonia. Intern Med 2001; 40: 873-877.

76 Clark BD, Vezza PR, Copeland C, Wilder AM, Abati A. Diagnostic sensitivity of bronchoalveolar lavage versus lung fine needle aspirate. Mod Pathol 2002; 15: 1259-1265.

77 Wimberley N, Faling LJ, Bartlett JG. A fiberoptic bronchoscopy technique to obtain uncontaminated lower airway secretions for bacterial culture. Am Rev Respir Dis 1979; 119: 337-343.

78 Wearden PD, Chendrasekhar A, Timberlake GA. Comparison of nonbronchoscopic techniques with bronchoscopic brushing in the diagnosis of ventilatorassociated pneumonia. J Trauma 1996; 41: 703-707.

79 Bello S, Tajada A, Chacon E, et al. "Blind" protected specimen brushing versus bronchoscopic techniques in the aetiolological diagnosis of ventilator-associated pneumonia. Eur Respir J 1996; 9: 1494-1499.

80 Thorpe JE, Baughman RP, Frame PT, Wesseler TA, Staneck JL. Bronchoalveolar lavage for diagnosing acute bacterial pneumonia. J Infect Dis 1987; 155: 855-861.

81 Meduri GU, Beals DH, Maijub AG, Baselski V. Protected bronchoalveolar lavage. A new bronchoscopic technique to retrieve uncontaminated distal airway secretions. Am Rev Respir Dis 1991; 143: 855-864.

82 Pereira Gomes JC, Pedreira JW Jr, Araujo EM, et al. Impact of BAL in the management of pneumonia with treatment failure: positivity of BAL culture under antibiotic therapy. Chest 2000; 118: 1739-1746.

83 Rasmussen TR, Korsgaard J, Moller JK, Sommer T, Kilian M. Quantitative culture of bronchoalveolar lavage fluid in community-acquired lower respiratory tract infections. Respir Med 2001; 95: 885-890.

84 Cantral DE, Tape TG, Reed EC, Spurzem JR, Rennard SI, Thompson AB. Quantitative culture of bronchoalveolar lavage fluid for the diagnosis of bacterial pneumonia. Am J Med 1993; 95: 601-607.

85 Chastre J, Viau F, Brun P, et al. Prospective evaluation of the protected specimen brush for the diagnosis of pulmonary infections in ventilated patients. Am Rev Respir Dis 1984; 130: 924-929.

86 Torres A, El Ebiary M. Bronchoscopic BAL in the diagnosis of ventilator-associated pneumonia. Chest 2000; 117: Suppl. 2, 198S-202S.

87 Rodriguez RM, Fancher ML, Phelps M, et al. An emergency department-based randomized trial of nonbronchoscopic bronchoalveolar lavage for early pathogen identification in severe community-acquired pneumonia Ann Emerg Med 2001; 38: 357-363.

88 Nagendra S, Bourbeau P, Brecher S, Dunne M, LaRocco M, Doern G. Sampling variability in the microbiological evaluation of expectorated sputa and endotracheal aspirates. J Clin Microbiol 2001; 39 ; 2344-2347.

89 Cooper GM, Jones JJ, Arbique JC, Flowerdew GJ, Forward KR. Intra and inter technologist variability in 
the quality assessment of respiratory tract specimens. Diagn Microbiol Infect Dis 2000; 37: 231-235.

90 Reed WW, Byrd GS, Gates RH Jr, Howard RS, Weaver MJ. Sputum gram's stain in community-acquired pneumococcal pneumonia. A meta-analysis. West J Med 1996; 165: 197-204.

91 Drew WL. Value of sputum culture in diagnosis of pneumococcal pneumonia. J Clin Microbiol 1977; 6: 62-65.

92 Roson B, Carratala J, Verdaguer R, Dorca J, Manresa F, Gudiol F. Prospective study of the usefulness of sputum Gram stain in the initial approach to communityacquired pneumonia requiring hospitalization. Clin Infect Dis 2000; 31: 869-874.

93 Ewig S, Schlochtermeier M, Goke N, Niederman MS. Applying sputum as a diagnostic tool in pneumonia: limited yield, minimal impact on treatment decisions. Chest 2002; 121: 1486-1492.

94 Geckler RW, Gremillion DH, McAllister CK, Ellenbogen C. Microscopic and bacteriological comparison of paired sputa and transtracheal aspirates. J Clin Microbiol 1977; 6: 396-399.

95 Parry CM, White RR, Ridgeway ER, Corkill JE, Smith GW. The reproducibility of sputum gram film interpretation. J Infect 2000; 41: 55-60.

96 Geckler RW, McAllister CK, Gremillion DH, Ellenbogen C. Clinical value of paired sputum and transtracheal aspirates in the initial management of pneumonia. Chest 1985; 87: 631-635.

97 Bartlett JG, Finegold SM. Bacteriology of expectorated sputum with quantitative culture and wash technique compared to transtracheal aspirates. Am Rev Respir Dis 1978; 117: 1019-1027.

98 Lentino JR, Lucks DA. Nonvalue of sputum culture in the management of lower respiratory tract infections. J Clin Microbiol 1987; 25: 758-762.

99 Barrett-Connor E. The nonvalue of sputum culture in the diagnosis of pneumococcal pneumonia. Am Rev Respir Dis 1971; 103: 845-848.

100 Murdoch DR, Laing RT, Mills GD, et al. Evaluation of a rapid immunochromatographic test for detection of Streptococcus pneumoniae antigen in urine samples from adults with community-acquired pneumonia. J Clin Microbiol 2001; 39: 3495-3498.

101 Dominguez J, Gali N, Blanco S, et al. Detection of Streptococcus pneumoniae antigen by a rapid immunochromatographic assay in urine samples. Chest 2001; 119: 243-249.

102 Burel E, Dufour P, Gauduchon V, Jarraud S, Etienne J. Evaluation of a rapid immunochromatographic assay for detection of Streptococcus pneumoniae antigen in urine samples. Eur J Clin Microbiol Infect Dis 2001; 20: 840-841.

103 Benson RF, Tang PW, Fields BS. Evaluation of the Binax and Biotest urinary antigen kits for detection of Legionnaires' disease due to multiple serogroups and species of Legionella. J Clin Microbiol 2000; 38: 2763-2765.

104 Dominguez JA, Gali N, Pedroso P, et al. Comparison of the Binax Legionella urinary antigen enzyme immunoassay (EIA) with the Biotest Legionella Urin antigen EIA for detection of Legionella antigen in both concentrated and nonconcentrated urine samples. J Clin Microbiol 1998; 36: 2718-2722.
105 Dominguez J, Gali N, Matas L, et al. Evaluation of a rapid immunochromatographic assay for the detection of Legionella antigen in urine samples. Eur J Clin Microbiol Infect Dis 1999; 18: 896-898.

106 Bernander S, Gastrin B, Lofgren S, Olinder-Nielsen AM. Legionella urinary antigen in early disease. Scand J Infect Dis 1994; 26: 777-778.

107 Yzerman EP, den Boer JW, Lettinga KD, Schellekens J, Dankert J, Peeters M. Sensitivity of three urinary antigen tests associated with clinical severity in a large outbreak of Legionnaires' disease in The Netherlands. J Clin Microbiol 2002; 40: 3232-3236.

108 Irmen KE, Kelleher JJ. Use of monoclonal antibodies for rapid diagnosis of respiratory viruses in a community hospital. Clin Diagn Lab Immunol 2000; 7: 396-403.

109 Reina J, Padilla E, Alonso F, Ruiz De Gopegui E, Munar M, Mari M. Evaluation of a new dot blot enzyme immunoassay (directigen flu $\mathrm{A}+\mathrm{B}$ ) for simultaneous and differential detection of influenza a and $B$ virus antigens from respiratory samples. J Clin Microbiol 2002; 40: 3515-3517.

110 Vikerfors T, Brodin G, Grandien M, Hirschberg L, Krook A, Pettersson CA. Detection of specific IgM antibodies for the diagnosis of Mycoplasma pneumoniae infections: a clinical evaluation. Scand J Infect Dis 1988; 20: 601-610.

111 Dorigo-Zetsma JW, Zaat SA, Wertheim-van Dillen PM, et al. Comparison of PCR, culture, and serological tests for diagnosis of Mycoplasma pneumoniae respiratory tract infection in children. J Clin Microbiol 1999; 37: 14-17.

112 Thacker WL, Talkington DF. Comparison of two rapid commercial tests with complement fixation for serologic diagnosis of Mycoplasma pneumoniae infections. J Clin Microbiol 1995; 33: 1212-1214.

113 Thacker WL, Talkington DF. Analysis of complement fixation and commercial enzyme immunoassays for detection of antibodies to Mycoplasma pneumoniae in human serum. Clin Diagn Lab Immunol 2000; 7: 778-780.

114 Ramirez JA, Ahkee S, Tolentino A, Miller RD, Summersgill JT. Diagnosis of Legionella pneumophila, Mycoplasma pneumoniae, or Chlamydia pneumoniae lower respiratory infection using the polymerase chain reaction on a single throat swab specimen. Diagn Microbiol Infect Dis 1996; 24: 7-14.

115 Grayston JT, Aldous MB, Easton A, et al. Evidence that Chlamydia pneumoniae causes pneumonia and bronchitis. J Infect Dis 1993; 168: 1231-1235.

116 Plouffe JF, File TM Jr, Breiman RF, et al. Reevaluation of the definition of Legionnaires' disease: use of the urinary antigen assay. Community Based Pneumonia Incidence Study Group. Clin Infect Dis 1995; 20: 1286-1291.

117 Petitjean J, Vabret A, Gouarin S, Freymuth F. Evaluation of four commercial immunoglobulin G (IgG)- and IgMspecific enzyme immunoassays for diagnosis of Mycoplasma pneumoniae infections. J Clin Microbiol 2002; 40: 165-171.

118 Herrmann B, Larsson C, Zweygberg BW. Simultaneous detection and typing of influenza viruses $A$ and $B$ by a nested reverse transcription-PCR: comparison to virus isolation and antigen detection by immunofluorescence 
and optical immunoassay (FLU OIA). J Clin Microbiol 2001; 39: 134-138.

119 Helms CM, Viner JP, Sturm RH, Renner ED, Johnson W. Comparative features of pneumococcal, mycoplasmal, and Legionnaires' disease pneumonias. Ann Intern Med 1979; 90: 543-547.

120 Woodhead MA, Macfarlane JT. Comparative clinical and laboratory features of legionella with pneumococcal and mycoplasma pneumonias. Br J Dis Chest 1987; 81: 133-139.

121 Kauppinen MT, Saikku P, Kujala P, Herva E, Syrjala H. Clinical picture of community-acquired Chlamydia pneumoniae pneumonia requiring hospital treatment: a comparison between chlamydial and pneumococcal pneumonia. Thorax 1996; 51: 185-189.

122 Farr BM, Kaiser DL, Harrison BD, Connolly CK. Prediction of microbial aetiology at admission to hospital for pneumonia from the presenting clinical features. British Thoracic Society Pneumonia Research Subcommittee. Thorax 1989; 44: 1031-1035.

123 Tang CM, Macfarlane JT. Early management of younger adults dying of community-acquired pneumonia. Respir Med 1993; 87: 289-294.

124 Meehan TP, Fine MJ, Krumholz HM, et al. Quality of care, process, and outcomes in elderly patients with pneumonia. JAMA 1997; 278: 2080-2084.

125 Simpson JC, Macfarlane JT, Watson J, Woodhead MA. A national confidential enquiry into community-acquired pneumonia deaths in young adults in England and Wales. British Thoracic Society Research Committee and Public Health Laboratory Service. Thorax 2000; 55: 1040-1045.

126 Leroy O, Santre C, Beuscart C, et al. A five-year study of severe community-acquired pneumonia with emphasis on prognosis in patients admitted to an intensive care unit. Intensive Care Med 1995; 21: 24-31.

127 Fang GD, Fine M, Orloff J, et al. New and emerging etiologies for community-acquired pneumonia with implications for therapy. A prospective multicenter study of 359 cases. Medicine (Baltimore) 1990; 69: 307-316.

128 Bohte R, van Furth R, van den Broek PJ. Aetiology of community-acquired pneumonia: a prospective study among adults requiring admission to hospital. Thorax 1995; 50: 543-547.

129 Gomez J, Banos V, Ruiz GJ, et al. Prospective study of epidemiology and prognostic factors in communityacquired pneumonia. Eur J Clin Microbiol Infect Dis 1996; 15: 556-560.

130 Steinhoff D, Lode H, Ruckdeschel G, et al. Chlamydia pneumoniae as a cause of community-acquired pneumonia in hospitalized patients in Berlin. Clin Infect Dis 1996; 22: 958-964.

131 Sopena N, Sabria M, Pedro-Botet ML, et al. Prospective study of community-acquired pneumonia of bacterial etiology in adults. Eur J Clin Microbiol Infect Dis 1999; 18: 852-858.

132 Socan M, Marinic-Fiser N, Kraigher A, Kotnik A, Logar M. Microbial aetiology of community-acquired pneumonia in hospitalised patients. Eur J Clin Microbiol Infect Dis 1999; 18: 777-782.
133 Falguera $M$, Sacristan $O$, Nogues A, et al. Nonsevere community-acquired pneumonia: correlation between cause and severity or comorbidity. Arch Intern Med 2001; 161: 1866-1872.

134 Arancibia F, Bauer TT, Ewig S, et al. Communityacquired pneumonia due to gram-negative bacteria and Pseudomonas aeruginosa: incidence, risk, and prognosis. Arch Intern Med 2002; 162: 1849-1858.

135 Torres A, Serra-Batlles J, Ferrer A, et al. Severe community-acquired pneumonia. Epidemiology and prognostic factors. Am Rev Respir Dis 1991; 144: 312-318.

136 Marik PE. The clinical features of severe communityacquired pneumonia presenting as septic shock. Norasept II Study Investigators. J Crit Care 2000; 15: 85-90.

137 Arancibia F, Ewig S, Martinez JA, et al. Antimicrobial treatment failures in patients with community-acquired pneumonia: causes and prognostic implications. Am J Respir Crit Care Med 2000; 162: 154-160.

138 Hatchette TF, Gupta R, Marrie TJ. Pseudomonas aeruginosa community-acquired pneumonia in previously healthy adults: case report and review of the literature. Clin Infect Dis 2000; 31: 1349-1356.

139 Riquelme R, Torres A, El Ebiary M, et al. Communityacquired pneumonia in the elderly: a multivariate analysis of risk and prognostic factors. Am J Respir Crit Care Med 1996; 154: 1450-1455.

140 Venkatesan P, Gladman J, Macfarlane JT, et al. A hospital study of community-acquired pneumonia in the elderly. Thorax 1990; 45: 254-258.

141 Metlay JP, Hofmann J, Cetron MS, et al. Impact of penicillin susceptibility on medical outcomes for adult patients with bacteremic pneumococcal pneumonia. Clin Infect Dis 2000; 30: 520-528.

142 Lynch IIIJP, Martinez FJ. Clinical relevance of macrolideresistant Streptococcus pneumoniae for communityacquired pneumonia. Clin Infect Dis 2002; 34: Suppl. 1, S27-S46.

143 Lonks JR, Garau J, Gomez L, et al. Failure of macrolide antibiotic treatment in patients with bacteremia due to erythromycin-resistant Streptococcus pneumoniae. Clin Infect Dis 2002; 35: 556-564.

144 Empey PE, Jennings HR, Thornton AC, Rapp RP, Evans ME. Levofloxacin failure in a patient with pneumococcal pneumonia. Ann Pharmacother 2001; 35: 687-690.

145 Ho PL, Tse WS, Tsang KW, et al. Risk factors for acquisition of levofloxacin-resistant Streptococcus pneumoniae: a case-control study. Clin Infect Dis 2001; 32: 701-707.

146 Davidson R, Cavalcanti R, Brunton JL, et al. Resistance to levofloxacin and failure of treatment of pneumococcal pneumonia. N Engl J Med 2002; 346: 747-750.

147 Pallares R, Linares J, Vadillo $M$, et al. Resistance to penicillin and cephalosporin and mortality from severe pneumococcal pneumonia in Barcelona, Spain. $N$ Engl J Med 1995; 333: 474-480.

148 Moroney JF, Fiore AE, Harrison LH, et al. Clinical outcomes of bacteremic pneumococcal pneumonia in the era of antibiotic resistance. Clin Infect Dis 2001; 33: 797-805. 
149 Felmingham D, Gruneberg RN. The Alexander Project 1996-1997: latest susceptibility data from this international study of bacterial pathogens from communityacquired lower respiratory tract infections. J Antimicrob Chemother 2000; 45: 191-203.

150 Fluit AC, Schmitz FJ, Jones ME, Acar J, Gupta R, Verhoef J. Antimicrobial resistance among communityacquired pneumonia isolates in Europe: first results from the SENTRY antimicrobial surveillance program 1997. SENTRY Participants Group. Int J Infect Dis 1999; 3: 153-156.

151 Pedersen G, Schonheyder HC, Steffensen FH, Sorensen HT. Risk of resistance related to antibiotic use before admission in patients with community-acquired bacteraemia. J Antimicrob Chemother 1999; 43: 119-126.

152 Benenson R, Magalski A, Cavanaugh S, Williams E. Effects of a pneumonia clinical pathway on time to antibiotic treatment, length of stay, and mortality. Acad Emerg Med 1999; 6: 1243-1248.

153 Ewig S, Seifert K, Kleinfeld T, Goke N, Schafer H. Management of patients with community-acquired pneumonia in a primary care hospital: a critical evaluation. Respir Med 2000; 94: 556-563.

154 Meehan TP, Weingarten SR, Holmboe ES, et al. A statewide initiative to improve the care of hospitalized pneumonia patients: The Connecticut Pneumonia Pathway Project. Am J Med 2001; 111: 203-210.

155 Hirani NA, Macfarlane JT. Impact of management guidelines on the outcome of severe community-acquired pneumonia. Thorax 1997; 52: 17-21.

156 Gleason PP, Meehan TP, Fine JM, Galusha DH, Fine MJ. Associations between initial antimicrobial therapy and medical outcomes for hospitalized elderly patients with pneumonia. Arch Intern Med 1999; 159: 2562-2572.

157 Burgess DS, Lewis JS. Effect of macrolides as part of initial empiric therapy on medical outcomes for hospitalized patients with community-acquired pneumonia. Clin Ther 2000; 22: 872-878.

158 Mundy LM, Oldach D, Auwaerter PG, et al. Implications for macrolide treatment in community-acquired pneumonia. Hopkins CAP Team. Chest 1998; 113: 1201-1206.

159 Cabellos C, Ariza J, Barreiro B, et al. Current usefulness of procaine penicillin in the treatment of pneumococcal pneumonia. Eur J Clin Microbiol Infect Dis 1998; 17: 265-268.

160 Pachon J, Prados MD, Capote F, Cuello JA, Garnacho J, Verano A. Severe community-acquired pneumonia. Etiology, prognosis, and treatment. Am Rev Respir Dis 1990; 142: 369-373.

161 Schentag JJ. Antimicrobial action and pharmacokinetics / pharmacodynamics: the use of AUIC to improve efficacy and avoid resistance. J Chemother 1999; 11: 426-439.

162 Ramirez JA, Srinath L, Ahkee S, Huang A, Raff MJ. Early switch from intravenous to oral cephalosporins in the treatment of hospitalized patients with communityacquired pneumonia. Arch Intern Med 1995; 155: 1273-1276.

163 Ramirez JA, Vargas S, Ritter GW, et al. Early switch from intravenous to oral antibiotics and early hospital discharge: a prospective observational study of 200 consecutive patients with community-acquired pneumonia. Arch Intern Med 1999; 159: 2449-2454.

164 Siegel RE, Halpern NA, Almenoff PL, Lee A, Cashin R, Greene JG. A prospective randomized study of inpatient iv. antibiotics for community-acquired pneumonia. The optimal duration of therapy. Chest 1996; 110: 965-971.

165 Dunn AS, Peterson KL, Schechter CB, Rabito P, Gotlin AD, Smith LG. The utility of an in-hospital observation period after discontinuing intravenous antibiotics. Am J Med 1999; 106: 6-10.

166 Ramirez JA, Bordon J. Early switch from intravenous to oral antibiotics in hospitalized patients with bacteremic community-acquired Streptococcus pneumoniae pneumonia. Arch Intern Med 2001; 161: 848-850.

167 Sanders WE Jr, Morris JF, Alessi P, et al. Oral ofloxacin for the treatment of acute bacterial pneumonia: use of a nontraditional protocol to compare experimental therapy with "usual care" in a multicenter clinical trial. Am J Med 1991; 91: 261-266.

168 Castro-Guardiola A, Viejo-Rodriguez AL, Soler-Simon S, et al. Efficacy and safety of oral and early-switch therapy for community-acquired pneumonia: a randomized controlled trial. Am J Med 2001; 111: 367-374.

169 Rhew DC, Riedinger MS, Sandhu M, Bowers C, Greengold N, Weingarten SR. A prospective, multicenter study of a pneumonia practice guideline. Chest 1998; 114: 115-119.

170 Rhew DC, Tu GS, Ofman J, Henning JM, Richards MS, Weingarten SR. Early switch and early discharge strategies in patients with community-acquired pneumonia: a meta-analysis. Arch Intern Med 2001; 161: 722-727.

171 File TM Jr, Segreti J, Dunbar L, et al. A multicenter, randomized study comparing the efficacy and safety of intravenous and/or oral levofloxacin versus ceftriaxone and/or cefuroxime axetil in treatment of adults with community-acquired pneumonia. Antimicrob Agents Chemother 1997; 41: 1965-1972.

172 Finch R, Schurmann D, Collins O, et al. Randomized controlled trial of sequential intravenous (i.v.) and oral moxifloxacin compared with sequential i.v. and oral coamoxiclav with or without clarithromycin in patients with community-acquired pneumonia requiring initial parenteral treatment. Antimicrob Agents Chemother 2002; 46: 1746-1754.

173 Samama MM, Cohen AT, Darmon JY, et al. A comparison of enoxaparin with placebo for the prevention of venous thromboembolism in acutely ill medical patients. Prophylaxis in Medical Patients with Enoxaparin Study Group. N Engl J Med 1999; 341: 793-800.

174 Confalonieri M, Potena A, Carbone G, Porta RD, Tolley EA, Umberto MG. Acute respiratory failure in patients with severe community-acquired pneumonia. A prospective randomized evaluation of noninvasive ventilation. Am J Respir Crit Care Med 1999; 160: 1585-1591.

175 Jolliet P, Abajo B, Pasquina P, Chevrolet JC. Non-invasive pressure support ventilation in severe communityacquired pneumonia. Intensive Care Med 2001; 27: 812-821.

176 International Consensus Conferences in Intensive Care Medicine: noninvasive positive pressure ventilation in 
acute respiratory failure. Am J Respir Crit Care Med 2001; 163: 283-291.

177 Nelson S, Heyder AM, Stone J, et al. A randomized controlled trial of filgrastim for the treatment of hospitalized patients with multilobar pneumonia. J Infect Dis 2000; 182: 970-973.

178 Metlay JP, Atlas SJ, Borowsky LH, Singer DE. Time course of symptom resolution in patients with community-acquired pneumonia. Respir Med 1998; 92: 1137-1142.

179 Macfarlane JT, Miller AC, Roderick Smith WH, Morris AH, Rose DH. Comparative radiographic features of community acquired Legionnaires' disease, pneumococcal pneumonia, mycoplasma pneumonia, and psittacosis. Thorax 1984; 39: 28-33.

180 Mittl RL Jr, Schwab RJ, Duchin JS, Goin JE, Albeida SM, Miller WT. Radiographic resolution of communityacquired pneumonia. Am J Respir Crit Care Med 1994; 149: 630-635.

181 Halm EA, Fine MJ, Marrie TJ, et al. Time to clinical stability in patients hospitalized with communityacquired pneumonia: implications for practice guidelines. JAMA 1998; 279: 1452-1457.

182 Halm EA, Fine MJ, Kapoor WN, Singer DE, Marrie TJ, Siu AL. Instability on hospital discharge and the risk of adverse outcomes in patients with pneumonia. Arch Intern Med 2002; 162: 1278-1284.

183 Fine MJ, Pratt HM, Obrosky DS, et al. Relation between length of hospital stay and costs of care for patients with community-acquired pneumonia. Am J Med 2000; 109: 378-385.

184 Menendez R, Ferrando D, Valles JM, Martinez E, Perpina M. Initial risk class and length of hospital stay in community-acquired pneumonia. Eur Respir J 2001; 18: 151-156.

185 McCormick D, Fine MJ, Coley CM, et al. Variation in length of hospital stay in patients with communityacquired pneumonia: are shorter stays associated with worse medical outcomes? Am J Med 1999; 107: 5-12.

186 Metersky ML, Tate JP, Fine MJ, Petrillo MK, Meehan TP. Temporal trends in outcomes of older patients with pneumonia. Arch Intern Med 2000; 160: 3385-3391.

187 Halm EA, Switzer GE, Mittman BS, Walsh MB, Chang CC, Fine MJ. What factors influence physicians' decisions to switch from intravenous to oral antibiotics for community-acquired pneumonia? J Gen Intern Med 2001; 16: 599-605.

188 Feinsilver SH, Fein AM, Niederman MS, Schultz DE, Faegenburg DH. Utility of fiberoptic bronchoscopy in nonresolving pneumonia. Chest 1990; 98: 1322-1326.

189 Celli BR, MacNee W. Standards for the diagnosis and treatment of patients with COPD: a summary of the ATS/ERS position paper. Eur Respir J 2004; 23: 932-946.

190 Garcia-Aymerich J, Monso E, Marrades RM, et al. Risk factors for hospitalization for a chronic obstructive pulmonary disease exacerbation. EFRAM study. Am J Respir Crit Care Med 2001; 164: 1002-1007.

191 Nouira S, Marghli S, Belghith M, Besbes L, Elatrous S, Abroug F. Once daily oral ofloxacin in chronic obstructive pulmonary disease exacerbation requiring mechanical ventilation: a randomised placebo-controlled trial. Lancet 2001; 358: 2020-2025.

192 McCrory DC, Brown C, Gelfand SE, Bach PB. Management of acute exacerbations of COPD: a summary and appraisal of published evidence. Chest 2001; 119: 1190-1209.

193 Monso E, Ruiz J, Rosell A, et al. Bacterial infection in chronic obstructive pulmonary disease. A study of stable and exacerbated outpatients using the protected specimen brush. Am J Respir Crit Care Med 1995; 152: 1316-1320.

194 Sethi S, Evans N, Grant BJ, Murphy TF. New strains of bacteria and exacerbations of chronic obstructive pulmonary disease. N Engl J Med 2002; 347: 465-471.

195 Monso E, Rosell A, Bonet G, et al. Risk factors for lower airway bacterial colonization in chronic bronchitis. Eur Respir J 1999; 13: 338-342.

196 Soler N, Torres A, Ewig S, et al. Bronchial microbial patterns in severe exacerbations of chronic obstructive pulmonary disease (COPD) requiring mechanical ventilation. Am J Respir Crit Care Med 1998; 157: 1498-1505.

197 Fagon JY, Chastre J, Trouillet JL, et al. Characterization of distal bronchial microflora during acute exacerbation of chronic bronchitis. Use of the protected specimen brush technique in 54 mechanically ventilated patients. Am Rev Respir Dis 1990; 142: 1004-1008.

198 Miravitlles M, Espinosa C, Fernandez-Laso E, Martos JA, Maldonado JA, Gallego M. Relationship between bacterial flora in sputum and functional impairment in patients with acute exacerbations of COPD. Study Group of Bacterial Infection in COPD. Chest 1999; 116: 40-46.

199 Eller J, Ede A, Schaberg T, Niederman MS, Mauch H, Lode $\mathrm{H}$. Infective exacerbations of chronic bronchitis: relation between bacteriologic etiology and lung function. Chest 1998; 113: 1542-1548.

200 Mogulkoc N, Karakurt S, Isalska B, et al. Acute purulent exacerbation of chronic obstructive pulmonary disease and Chlamydia pneumoniae infection. Am J Respir Crit Care Med 1999; 160: 349-353.

201 Lieberman D, Ben Yaakov M, Lazarovich Z, Ohana B, Boldur I. Chlamydia pneumoniae infection in acute exacerbations of chronic obstructive pulmonary disease: analysis of 250 hospitalizations. Eur J Clin Microbiol Infect Dis 2001; 20: 698-704.

202 Seemungal T, Harper-Owen R, Bhowmik A, et al. Respiratory viruses, symptoms, and inflammatory markers in acute exacerbations and stable chronic obstructive pulmonary disease. Am J Respir Crit Care Med 2001; 164: 1618-1623.

203 Ewig S, Soler N, Gonzalez J, Celis R, El Ebiary M, Torres A. Evaluation of antimicrobial treatment in mechanically ventilated patients with severe chronic obstructive pulmonary disease exacerbations. Crit Care Med 2000; 28: 692-697.

204 Anzueto A, Niederman MS, Haverstock DC, Tillotson GS Efficacy of ciprofloxacin and clarithromycin in acute bacterial exacerbations of complicated chronic bronchitis: interim analysis. Bronchitis Study Group. Clin Ther 1997; 19: 989-1001. 
205 Adams SG, Melo J, Luther M, Anzueto A. Antibiotics are associated with lower relapse rates in outpatients with acute exacerbations of COPD. Chest 2000; 117: 1345-1352.

206 Pang JA, Cheng A, Chan HS, Poon D, French G. The bacteriology of bronchiectasis in Hong Kong investigated by protected catheter brush and bronchoalveolar lavage. Am Rev Respir Dis 1989; 139: 14-17.

207 Cabello H, Torres A, Celis R, et al. Bacterial colonization of distal airways in healthy subjects and chronic lung disease: a bronchoscopic study. Eur Respir J 1997; 10: 1137-1144.

208 Angrill J, Agusti C, de Celis R, et al. Bacterial colonisation in patients with bronchiectasis: microbiological pattern and risk factors. Thorax 2002; 57: 15-19.

209 Pela R, Marchesani F, Agostinelli C, et al. Airways microbial flora in COPD patients in stable clinical conditions and during exacerbations: a bronchoscopic investigation. Monaldi Arch Chest Dis 1998; 53: 262-267.

210 Groeneveld K, van Alphen L, Eijk PP, Visschers G, Jansen HM, Zanen HC. Endogenous and exogenous reinfections by Haemophilus influenzae in patients with chronic obstructive pulmonary disease: the effect of antibiotic treatment on persistence. J Infect Dis 1990; 161: 512-517.

211 Hill AT, Bayley D, Stockley RA. The interrelationship of sputum inflammatory markers in patients with chronic bronchitis. Am J Respir Crit Care Med 1999; 160: 893-898.

212 Ball P, Make B. Acute exacerbations of chronic bronchitis: an international comparison. Chest 1998; 113: Suppl. 3, 199S-204S.

213 Biebuyck XA. Comparison of azithromycin and coamoxiclav in the treatment of acute tracheobronchitis and acute infectious exacerbations of chronic bronchitis in adults. Azithromycin Study Group. J Int Med Res 1996; 24: 407-418.

214 Aubier M, Aldons PM, Leak A, et al. Telithromycin is as effective as amoxicillin/clavulanate in acute exacerbations of chronic bronchitis. Respir Med 2002; 96: 862-871.

215 Blondeau JM, Tillotson GS. Antimicrobial susceptibility patterns of respiratory pathogens--a global perspective. Semin Respir Infect 2000; 15: 195-207.

216 Langan CE, Cranfield R, Breisch S, Pettit R. Randomized, double-blind study of grepafloxacin versus amoxycillin in patients with acute bacterial exacerbations of chronic bronchitis. J Antimicrob Chemother 1997; 40: Suppl. A, 63-72.

217 Wilson R, Kubin R, Ballin I, et al. Five day moxifloxacin therapy compared with 7 day clarithromycin therapy for the treatment of acute exacerbations of chronic bronchitis. J Antimicrob Chemother 1999; 44: 501-513.

218 Lorenz J, Thate-Waschke IM, Mast O, et al. Treatment outcomes in acute exacerbations of chronic bronchitis: comparison of macrolides and moxifloxacin from the patient perspective. J Int Med Res 2001; 29: 74-86.

219 Shah PM, Maesen FP, Dolmann A, Vetter N, Fiss E, Wesch R. Levofloxacin versus cefuroxime axetil in the treatment of acute exacerbation of chronic bronchitis: results of a randomized, double-blind study. J Antimicrob Chemother 1999; 43: 529-539.
220 Grossman RF. Cost-effective therapy for acute exacerbations of chronic bronchitis. Semin Respir Infect 2000; 15: 71-81.

221 Miravitlles M. [Treatment failure of acute exacerbations of chronic obstructive airways disease risk factors and clinical relevance]. Med Clin (Barc) 2002; 119: 304-314.

222 Dewan NA, Rafique S, Kanwar B, et al. Acute exacerbation of COPD: factors associated with poor treatment outcome. Chest 2000; 117: 662-671.

223 Chodosh S, DeAbate CA, Haverstock D, Aneiro L, Church D. Short-course moxifloxacin therapy for treatment of acute bacterial exacerbations of chronic bronchitis. The Bronchitis Study Group. Respir Med 2000; 94: 18-27.

224 DeAbate CA, Mathew CP, Warner JH, Heyd A, Church D. The safety and efficacy of short course (5-day) moxifloxacin vs. azithromycin in the treatment of patients with acute exacerbation of chronic bronchitis. Respir Med 2000; 94: 1029-1037.

225 Pasteur MC, Helliwell SM, Houghton SJ, et al. An investigation into causative factors in patients with bronchiectasis. Am J Respir Crit Care Med 2000; 162 1277-1284.

226 Ho PL, Chan KN, Ip MS, et al. The effect of Pseudomonas aeruginosa infection on clinical parameters in steady-state bronchiectasis. Chest 1998; 114: 1594-1598.

227 Evans SA, Turner SM, Bosch BJ, Hardy CC, Woodhead MA. Lung function in bronchiectasis: the influence of Pseudomonas aeruginosa. Eur Respir J 1996; 9: 1601-1604.

228 Miszkiel KA, Wells AU, Rubens MB, Cole PJ, Hansell DM. Effects of airway infection by Pseudomonas aeruginosa: a computed tomographic study. Thorax 1997; 52: 260-264.

229 Angrill J, Agusti C, de Celis R, et al. Bronchial inflammation and colonization in patients with clinically stable bronchiectasis. Am J Respir Crit Care Med 2001; 164: 1628-1632.

230 Shah PL, Mawdsley S, Nash K, Cullinan P, Cole PJ, Wilson R. Determinants of chronic infection with Staphylococcus aureus in patients with bronchiectasis. Eur Respir J 1999; 14: 1340-1344.

231 Mitchell JL, Hill SL. Immune response to Haemophilus parainfluenzae in patients with chronic obstructive lung disease. Clin Diagn Lab Immunol 2000; 7: 25-30.

232 Tsang KW, Chan WM, Ho PL, Chan K, Lam WK, Ip MS. A comparative study on the efficacy of levofloxacin and ceftazidime in acute exacerbation of bronchiectasis. Eur Respir J 1999; 14: 1206-1209.

233 Foxwell AR, Cripps AW, Dear KB. Haemophilus influenzae oral whole cell vaccination for preventing acute exacerbations of chronic bronchitis. Cochrane Database Syst Rev 2003; 3: CD001958.

234 Orcel B, Delclaux B, Baud M, Derenne JP. Oral immunization with bacterial extracts for protection against acute bronchitis in elderly institutionalized patients with chronic bronchitis. Eur Respir J 1994; 7: 446-452.

235 Collet JP, Shapiro P, Ernst P, Renzi T, Ducruet T, Robinson A. Effects of an immunostimulating agent on acute exacerbations and hospitalizations in patients with chronic obstructive pulmonary disease. The PARI-IS 
Study Steering Committee and Research Group. Prevention of Acute Respiratory Infection by an Immunostimulant. Am J Respir Crit Care Med 1997; 156: 1719-1724.

236 Johnston RN, McNeill RS, Smith DH, et al. Five-year winter chemoprophylaxis for chronic bronchitis. BMJ 1969; 4: 265-269.

237 Value of chemoprophylaxis and chemotherapy in early chronic bronchitis. A report to the Medical Research Council by their working party on trials of chemotherapy in early chronic bronchitis. BMJ 1966; 5499: 1317-1322.

238 Pridie RB, Datta N, Massey DG, Poole GW, Schneeweiss J, Stradling P. A trial of continuous winter chemotherapy in chronic bronchitis. Lancet 1960; 2: 723-727.

239 Gadomski AM. Potential interventions for preventing pneumonia among young children: lack of effect of antibiotic treatment for upper respiratory infections. Pediatr Infect Dis J 1993; 12: 115-120.

240 Arroll B, Kenealy T. Antibiotics for the common cold. Cochrane Database Syst Rev 2000; 2: CD000247.

241 Jefferson TO, Tyrrell D. Antivirals for the common cold. Cochrane Database Syst Rev 2001; 3: CD002743.

242 BTS guidelines for the management of chronic obstructive pulmonary disease. The COPD Guidelines Group of the Standards of Care Committee of the BTS. Thorax 1997; 52: Suppl. 5, S1-28.

243 Pauwels RA, Buist AS, Calverley PM, Jenkins CR, Hurd SS. Global strategy for the diagnosis, management, and prevention of chronic obstructive pulmonary disease. NHLBI/WHO Global Initiative for Chronic Obstructive Lung Disease (GOLD) Workshop summary. Am J Respir Crit Care Med 2001; 163: 1256-1276.

244 Jones AP, Rowe BH. Bronchopulmonary hygiene physical therapy for chronic obstructive pulmonary disease and bronchiectasis. Cochrane Database Syst Rev 2000; 2: CD000045.

245 Hayden FG, Atmar RL, Schilling M, et al. Use of the selective oral neuraminidase inhibitor oseltamivir to prevent influenza. $N$ Engl J Med 1999; 341: 1336-1343.

246 Hayden FG, Gubareva LV, Monto AS, et al. Inhaled zanamivir for the prevention of influenza in families. Zanamivir Family Study Group. N Engl J Med 2000; 343: 1282-1289.

247 Monto AS, Robinson DP, Herlocher ML, Hinson JM Jr, Elliott MJ, Crisp A. Zanamivir in the prevention of influenza among healthy adults: a randomized controlled trial. JAMA 1999; 282: 31-35.

248 Monto AS, Pichichero ME, Blanckenberg SJ, et al. Zanamivir prophylaxis: an effective strategy for the prevention of influenza types A and B within households. J Infect Dis 2002; 186: 1582-1588.

249 Jefferson T, Demicheli V, Deeks J, Rivetti D. Neuraminidase inhibitors for preventing and treating influenza in healthy adults. Cochrane Database Syst Rev 2000; 2: CD001265.

250 Poole PJ, Black PN. Mucolytic agents for chronic bronchitis or chronic obstructive pulmonary disease. Cochrane Database Syst Rev 2000; 2: CD001287.

251 Stey C, Steurer J, Bachmann S, Medici TC, Tramer MR. The effect of oral $\mathrm{N}$-acetylcysteine in chronic bronchitis: a quantitative systematic review. Eur Respir J 2000; 16 : 253-262.

252 Crockett AJ, Cranston JM, Latimer KM, Alpers JH. Mucolytics for bronchiectasis. Cochrane Database Syst Rev 2001; 1: CD001289.

253 Melchart D, Linde K, Fischer P, Kaesmayr J. Echinacea for preventing and treating the common cold. Cochrane Database Syst Rev 2000; 2: CD000530.

254 Belongia EA, Berg R, Liu K. A randomized trial of zinc nasal spray for the treatment of upper respiratory illness in adults. Am J Med 2001; 111: 103-108.

255 Marshall I. Zinc for the common cold. Cochrane Database Syst Rev 2000; 2: CD001364.

256 Jackson JL, Lesho E, Peterson C. Zinc and the common cold: a meta-analysis revisited. J Nutr 2000; 130: Suppl. 5, 1512S-1515S.

257 Vickers AJ, Smith C. Homoeopathic oscillococcinum for preventing and treating influenza and influenza-like syndromes. Cochrane Database Syst Rev 2000; 2: CD001957.

258 Beyer WE, Palache AM, de Jong JC, Osterhaus AD. Coldadapted live influenza vaccine versus inactivated vaccine: systemic vaccine reactions, local and systemic antibody response, and vaccine efficacy. A meta-analysis. Vaccine 2002; 20: 1340-1353.

259 Gravenstein S, Drinka P, Duthie EH, et al. Efficacy of an influenza hemagglutinin-diphtheria toxoid conjugate vaccine in elderly nursing home subjects during an influenza outbreak. J Am Geriatr Soc 1994; 42: 245-251.

260 Keren G, Segev S, Morag A, Zakay-Rones Z, Barzilai A, Rubinstein E. Failure of influenza vaccination in the aged. J Med Virol 1988; 25: 85-89.

261 Iorio AM, Zei T, Neri M, Campitelli L, Castrucci MR, Donatelli I. Immunization of elderly volunteers with the 1988-89 inactivated whole influenza vaccine: assessment of antibody responses by haemagglutination inhibition and single radial haemolysis tests. Eur J Epidemiol 1992; 8: 491-497.

262 Govaert TM, Sprenger MJ, Dinant GJ, Aretz K, Masurel N, Knottnerus JA. Immune response to influenza vaccination of elderly people. A randomized double-blind placebo-controlled trial. Vaccine 1994; 12: 1185-1189.

263 Bernstein E, Kaye D, Abrutyn E, Gross P, Dorfman M, Murasko DM. Immune response to influenza vaccination in a large healthy elderly population. Vaccine 1999; 17: 82-94.

264 Roos-Van Eijndhoven DG, Cools HJ, Westendorp RG, Cate-Hoek AJ, Knook DL, Remarque EJ. Randomized controlled trial of seroresponses to double dose and booster influenza vaccination in frail elderly subjects. J Med Virol 2001; 63: 293-298.

265 Margolis KL, Nichol KL, Poland GA, Pluhar RE. Frequency of adverse reactions to influenza vaccine in the elderly. A randomized, placebo-controlled trial. JAMA 1990; 264: 1139-1141.

266 Govaert TM, Dinant GJ, Aretz K, Masurel N, Sprenger MJ, Knottnerus JA. Adverse reactions to influenza vaccine in elderly people: randomised double blind placebo controlled trial. BMJ 1993; 307: 988-990.

267 Allsup SJ, Gosney M, Regan M, Haycox A, Fear S, Johnstone FC. Side effects of influenza vaccination in 
healthy older people: a randomised single-blind placebocontrolled trial. Gerontology 2001; 47: 311-314.

268 The safety of inactivated influenza vaccine in adults and children with asthma. N Engl J Med 2001; 345: 1529-1536.

269 al-Mazrou A, Scheifele DW, Soong T, Bjornson G. Comparison of adverse reactions to whole-virion and split-virion influenza vaccines in hospital personnel. CMAJ 1991; 145: 213-218.

270 Honkanen PO, Keistinen T, Kivela SL. Reactions following administration of influenza vaccine alone or with pneumococcal vaccine to the elderly. Arch Intern Med 1996; 156: 205-208.

271 Nichol KL, Mendelman PM, Mallon KP, et al. Effectiveness of live, attenuated intranasal influenza virus vaccine in healthy, working adults: a randomized controlled trial. JAMA 1999; 282: 137-144.

272 Demicheli V, Rivetti D, Deeks JJ, Jefferson TO. Vaccines for preventing influenza in healthy adults. Cochrane Database Syst Rev 2000; 2: CD001269.

273 Govaert TM, Thijs CT, Masurel N, Sprenger MJ, Dinant GJ, Knottnerus JA. The efficacy of influenza vaccination in elderly individuals. A randomized doubleblind placebo-controlled trial. JAMA 1994; 272: 1661-1665.

274 Gross PA, Hermogenes AW, Sacks HS, Lau J, Levandowski RA. The efficacy of influenza vaccine in elderly persons. A meta-analysis and review of the literature. Ann Intern Med 1995; 123: 518-527.

275 Nichol KL, Margolis KL, Wuorenma J, Von Sternberg T. The efficacy and cost effectiveness of vaccination against influenza among elderly persons living in the community. N Engl J Med 1994; 331: 778-784.

276 Christenson B, Lundbergh P, Hedlund J, Ortqvist A. Effects of a large-scale intervention with influenza and 23 -valent pneumococcal vaccines in adults aged 65 years or older: a prospective study. Lancet 2001; 357: 1008-1011.

277 Nordin J, Mullooly J, Poblete S, et al. Influenza vaccine effectiveness in preventing hospitalizations and deaths in persons 65 years or older in Minnesota, New York, and Oregon: data from 3 health plans. J Infect Dis 2001; 184: 665-670.

278 Puig-Barbera J, Marquez-Calderon S, Masoliver-Fores A, et al. Reduction in hospital admissions for pneumonia in non-institutionalised elderly people as a result of influenza vaccination: a case-control study in Spain. J Epidemiol Community Health 1997; 51: 526-530.

279 Crocetti E, Arniani S, Bordoni F, Maciocco G, Zappa M, Buiatti E. Effectiveness of influenza vaccination in the elderly in a community in Italy. Eur J Epidemiol 2001; 17: 163-168.

280 Nichol KL, Wuorenma J, Von Sternberg T. Benefits of influenza vaccination for low-, intermediate-, and highrisk senior citizens. Arch Intern Med 1998; 158: 1769-1776.

281 Hak E, Nordin J, Wei F, et al. Influence of high-risk medical conditions on the effectiveness of influenza vaccination among elderly members of 3 large managedcare organizations. Clin Infect Dis 2002; 35: 370-377.

282 Treanor JJ, Mattison HR, Dumyati G, et al. Protective efficacy of combined live intranasal and inactivated influenza A virus vaccines in the elderly. Ann Intern Med 1992; 117: 625-633.
283 Monto AS, Hornbuckle K, Ohmit SE. Influenza vaccine effectiveness among elderly nursing home residents: a cohort study. Am J Epidemiol 2001; 154: 155-160.

284 Deguchi Y, Takasugi Y, Tatara K. Efficacy of influenza vaccine in the elderly in welfare nursing homes: reduction in risks of mortality and morbidity during an influenza A (H3N2) epidemic. J Med Microbiol 2000; 49: 553-556.

285 Potter J, Stott DJ, Roberts MA, et al. Influenza vaccination of health care workers in long-term-care hospitals reduces the mortality of elderly patients. I Infect Dis 1997; 175: 1-6.

286 Carman WF, Elder AG, Wallace LA, et al. Effects of influenza vaccination of health-care workers on mortality of elderly people in long-term care: a randomised controlled trial. Lancet 2000; 355: 93-97.

287 Bridges CB, Thompson WW, Meltzer MI, et al. Effectiveness and cost-benefit of influenza vaccination of healthy working adults: a randomized controlled trial. JAMA 2000; 284: 1655-1663.

288 Nichol KL, Lind A, Margolis KL, et al. The effectiveness of vaccination against influenza in healthy, working adults. N Engl J Med 1995; 333: 889-893.

289 Nichol KL. Cost-benefit analysis of a strategy to vaccinate healthy working adults against influenza. Arch Intern Med 2001; 161: 749-759.

290 Mullooly JP, Bennett MD, Hornbrook MC, et al. Influenza vaccination programs for elderly persons: costeffectiveness in a health maintenance organization. Ann Intern Med 1994; 121: 947-952.

291 Scott WG, Scott HM. Economic evaluation of vaccination against influenza in New Zealand. Pharmacoeconomics 1996; 9: 51-60.

292 Hak E, van Essen GA, Buskens E, Stalman W, de Melker RA. Is immunising all patients with chronic lung disease in the community against influenza cost effective? Evidence from a general practice based clinical prospective cohort study in Utrecht, The Netherlands. J Epidemiol Community Health 1998; 52: 120-125.

293 Muennig PA, Khan K. Cost-effectiveness of vaccination versus treatment of influenza in healthy adolescents and adults. Clin Infect Dis 2001; 33: 1879-1885.

294 Beyer WE, de Bruijn IA, Palache AM, Westendorp RG, Osterhaus AD. Protection against influenza after annually repeated vaccination: a meta-analysis of serologic and field studies. Arch Intern Med 1999; 159: 182-188.

295 Buxton JA, Skowronski DM, $\mathrm{Ng} \mathrm{H}$, et al. Influenza revaccination of elderly travellers: antibody response to single influenza vaccination and revaccination at 12 weeks. J Infect Dis 2001; 184: 188-191.

296 Bridges CB, Fukuda K, Cox NJ, Singleton JA. Prevention and control of influenza. Recommendations of the Advisory Committee on Immunization Practices (ACIP). MMWR Recomm Rep 2001; 50: 1-44.

297 Lipsky BA, Boyko EJ, Inui TS, Koepsell TD. Risk factors for acquiring pneumococcal infections. Arch Intern Med 1986; 146: 2179-2185.

298 Nuorti JP, Butler JC, Farley MM, et al. Cigarette smoking and invasive pneumococcal disease. Active Bacterial Core Surveillance Team. N Engl J Med 2000; 342: 681-689. 
299 Prevention of pneumococcal disease: recommendations of the Advisory Committee on Immunization Practices (ACIP). MMWR Recomm Rep 1997; 46: 1-24.

300 Hedlund JU, Kalin ME, Ortqvist AB, Henrichsen J. Antibody response to pneumococcal vaccine in middleaged and elderly patients recently treated for pneumonia. Arch Intern Med 1994; 154: 1961-1965.

301 Sankilampi U, Honkanen PO, Bloigu A, Herva E, Leinonen M. Antibody response to pneumococcal capsular polysaccharide vaccine in the elderly. I Infect Dis 1996; 173: 387-393.

302 Carson PJ, Nichol KL, O'Brien J, Hilo P, Janoff EN. Immune function and vaccine responses in healthy advanced elderly patients. Arch Intern Med 2000; 160: 2017-2024.

303 Rubins JB, Puri AK, Loch J, et al. Magnitude, duration, quality, and function of pneumococcal vaccine responses in elderly adults. I Infect Dis 1998; 178: 431-440.

304 Rubins JB, Alter M, Loch J, Janoff EN. Determination of antibody responses of elderly adults to all 23 capsular polysaccharides after pneumococcal vaccination. Infect Immun 1999; 67: 5979-5984.

305 Romero-Steiner S, Musher DM, Cetron MS, et al. Reduction in functional antibody activity against Streptococcus pneumoniae in vaccinated elderly individuals highly correlates with decreased IgG antibody avidity. Clin Infect Dis 1999; 29: 281-288.

306 Sankilampi U, Honkanen PO, Bloigu A, Leinonen M. Persistence of antibodies to pneumococcal capsular polysaccharide vaccine in the elderly. J Infect Dis 1997; 176: 1100-1104.

307 Konradsen HB. Quantity and avidity of pneumococcal antibodies before and up to five years after pneumococcal vaccination of elderly persons. Clin Infect Dis 1995; 21: 616-620.

308 Hedlund J, Ortqvist A, Konradsen HB, Kalin M. Recurrence of pneumonia in relation to the antibody response after pneumococcal vaccination in middle-aged and elderly adults. Scand J Infect Dis 2000; 32: 281-286.

309 Powers DC, Anderson EL, Lottenbach K, Mink CM. Reactogenicity and immunogenicity of a protein-conjugated pneumococcal oligosaccharide vaccine in older adults. J Infect Dis 1996; 173: 1014-1018.

310 Shelly MA, Jacoby H, Riley GJ, Graves BT, Pichichero M, Treanor JJ. Comparison of pneumococcal polysaccharide and CRM197-conjugated pneumococcal oligosaccharide vaccines in young and elderly adults. Infect Immun 1997; 65: 242-247.

311 Musher DM, Groover JE, Watson DA, RodriguezBarradas MC, Baughn RE. IgG responses to proteinconjugated pneumococcal capsular polysaccharides in persons who are genetically incapable of responding to unconjugated polysaccharides. Clin Infect Dis 1998; 27: 1487-1490.

312 Jonsson S, Vidarsson G, Valdimarsson H, Schiffman G, Schneerson R, Jonsdottir I. Vaccination of COPD patients with a pneumococcus type $6 \mathrm{~B}$ tetanus toxoid conjugate vaccine. Eur Respir J 2002; 20: 813-818.

313 Jackson LA, Benson P, Sneller VP, et al. Safety of revaccination with pneumococcal polysaccharide vaccine. JAMA 1999; 281: 243-248.
314 Fine MJ, Smith MA, Carson CA, et al. Efficacy of pneumococcal vaccination in adults. A meta-analysis of randomized controlled trials. Arch Intern Med 1994; 154: 2666-2677.

315 Hutchison BG, Oxman AD, Shannon HS, Lloyd S, Altmayer CA, Thomas K. Clinical effectiveness of pneumococcal vaccine. Meta-analysis. Can Fam Physician 1999; 45: 2381-2393.

316 Moore RA, Wiffen PJ, Lipsky BA. Are the pneumococcal polysaccharide vaccines effective? Meta-analysis of the prospective trials. BMC Fam Pract 2000; 1: 1.

317 Cornu C, Yzebe D, Leophonte P, Gaillat J, Boissel JP, Cucherat M. Efficacy of pneumococcal polysaccharide vaccine in immunocompetent adults: a meta-analysis of randomized trials. Vaccine 2001; 19: 4780-4790.

318 Watson L, Wilson BJ, Waugh N. Pneumococcal polysaccharide vaccine: a systematic review of clinical effectiveness in adults. Vaccine 2002; 20: 2166-2173.

319 Austrian R, Douglas RM, Schiffman G, et al. Prevention of pneumococcal pneumonia by vaccination. Trans Assoc Am Physicians 1976; 89: 184-194.

320 Smit P, Oberholzer D, Hayden-Smith S, Koornhof HJ, Hilleman MR. Protective efficacy of pneumococcal polysaccharide vaccines. JAMA 1977; 238: 2613-2616.

321 Ortqvist A, Hedlund J, Burman LA, et al. Randomised trial of 23-valent pneumococcal capsular polysaccharide vaccine in prevention of pneumonia in middle-aged and elderly people. Swedish Pneumococcal Vaccination Study Group. Lancet 1998; 351: 399-403.

322 Honkanen PO, Keistinen T, Miettinen L, et al. Incremental effectiveness of pneumococcal vaccine on simultaneously administered influenza vaccine in preventing pneumonia and pneumococcal pneumonia among persons aged 65 years or older. Vaccine 1999; 17: 2493-2500.

323 Forrester HL, Jahnigen DW, LaForce FM. Inefficacy of pneumococcal vaccine in a high-risk population. Am Med 1987; 83: 425-430.

324 Sims RV, Steinmann WC, McConville JH, King LR, Zwick WC, Schwartz JS. The clinical effectiveness of pneumococcal vaccine in the elderly. Ann Intern Med 1988; 108: 653-657.

325 Shapiro ED, Berg AT, Austrian R, et al. The protective efficacy of polyvalent pneumococcal polysaccharide vaccine. N Engl J Med 1991; 325: 1453-1460.

326 Butler JC, Breiman RF, Campbell JF, Lipman HB, Broome CV, Facklam RR. Pneumococcal polysaccharide vaccine efficacy. An evaluation of current recommendations. JAMA 1993; 270: 1826-1831.

327 Farr BM, Johnston BL, Cobb DK, et al. Preventing pneumococcal bacteremia in patients at risk. Results of a matched case-control study. Arch Intern Med 1995; 155: 2336-2340.

328 Nichol KL, Baken L, Wuorenma J, Nelson A. The health and economic benefits associated with pneumococcal vaccination of elderly persons with chronic lung disease. Arch Intern Med 1999; 159: 2437-2442.

329 Vold PP, Owens DK. Cost-effectiveness of the pneumococcal vaccine in the United States Navy and Marine Corps. Clin Infect Dis 2000; 30: 157-164. 
330 Sisk JE, Moskowitz AJ, Whang W, et al. Cost-effectiveness of vaccination against pneumococcal bacteremia among elderly people. JAMA 1997; 278: 1333-1339.

331 Ament A, Baltussen R, Duru G, et al. Cost-effectiveness of pneumococcal vaccination of older people: a study in 5 western European countries. Clin Infect Dis 2000; 31: 444-450.

332 Postma MJ, Heijnen ML, Jager JC. Cost-effectiveness analysis of pneumococcal vaccination for elderly individuals in The Netherlands. Pharmacoeconomics 2001; 19: 215-222.

333 Rutherford EJ, Livengood J, Higginbotham $\mathrm{M}$, et al. Efficacy and safety of pneumococcal revaccination after splenectomy for trauma. J Trauma 1995; 39: 448-452.

334 Rehmet S, Ammon A, Pfaff G, Bocter N, Petersen LR. Cross-sectional study on influenza vaccination, Germany, 1999-2000. Emerg Infect Dis 2002; 8: 1442-1447.

335 Opstelten W, Hak E, Verheij TJ, van Essen GA. Introducing a pneumococcal vaccine to an existing influenza immunization program: vaccination rates and predictors of noncompliance. Am J Med 2001; 111: 474-479.

336 Shefer A, Briss P, Rodewald L, et al. Improving immunization coverage rates: an evidence-based review of the literature. Epidemiol Rev 1999; 21: 96-142.

337 Siriwardena AN, Rashid A, Johnson MR, Dewey ME. Cluster randomised controlled trial of an educational outreach visit to improve influenza and pneumococcal immunisation rates in primary care. Br J Gen Pract 2002; 52: 735-740.

338 Sarnoff R, Rundall T. Meta-analysis of effectiveness of interventions to increase influenza immunization rates among high-risk population groups. Med Care Res Rev 1998; 55: 432-456.
339 Nexoe J, Kragstrup J, Ronne T. Impact of postal invitations and user fee on influenza vaccination rates among the elderly. A randomized controlled trial in general practice. Scand J Prim Health Care 1997; 15: 109-112.

340 Honkanen PO, Keistinen T, Kivela SL. The impact of vaccination strategy and methods of information on influenza and pneumococcal vaccination coverage in the elderly population. Vaccine 1997; 15: 317-320.

341 Satterthwaite P. A randomised intervention study to examine the effect on immunisation coverage of making influenza vaccine available at no cost. N Z Med J 1997; 110: 58-60.

342 Krieger JW, Castorina JS, Walls ML, Weaver MR, Ciske S. Increasing influenza and pneumococcal immunization rates: a randomized controlled study of a senior centerbased intervention. Am J Prev Med 2000; 18: 123-131.

343 Arthur AJ, Matthews RJ, Jagger C, Clarke M, Hipkin A, Bennison DP. Improving uptake of influenza vaccination among older people: a randomised controlled trial. $\mathrm{Br} \mathrm{J}$ Gen Pract 2002; 52: 717-718, 720-722.

344 Shevlin JD, Summers-Bean C, Thomas D, Whitney CG, Todd D, Ray SM. A systematic approach for increasing pneumococcal vaccination rates at an inner-city public hospital. Am J Prev Med 2002; 22: 92-97.

345 Stevenson KB, McMahon JW, Harris J, Hillman JR, Helgerson SD. Increasing pneumococcal vaccination rates among residents of long-term--care facilities: provider-based improvement strategies implemented by peer-review organizations in four western states. Infect Control Hosp Epidemiol 2000; 21: 705-710.

346 Dey P, Halder S, Collins S, Benons L, Woodman C. Promoting uptake of influenza vaccination among health care workers: a randomized controlled trial. J Public Health Med 2001; 23: 346-348. 